\title{
AERODYNAMIC AND STRUCTURAL DESIGN OF A SMALL NONPLANAR WING UAV
}

\author{
Thesis \\ Submitted to \\ The School of Engineering of the \\ UNIVERSITY OF DAYTON
}

In partial fulfillment of the Requirements for

The Degree of

Master of Science in Aerospace Engineering

\begin{tabular}{c} 
By \\
Giuseppe Lando \\
Dayton, Ohio \\
May 2008 \\
unIVERsITY of \\
\hline DAYTON \\
\hline Din
\end{tabular} 


\begin{abstract}
AERODYNAMIC AND STRUCTURAL DESIGN OF A SMALL NONPLANAR WING UAV
\end{abstract}

Name: Landolfo, Giuseppe

University of Dayton

Advisor: Dr. Aaron Altman

The overall air vehicle performance of a multiple lifting surface configuration has been studied with respect to both structural and aerodynamic considerations for a candidate mission similar to that of the AeroVironment Raven. The configuration studied is a biplane joined at the tips with endplates. More specifically, this study aims to determine if this particular nonplanar wing concept can meet the requirements of the mission for a small Reconnaissance, Surveillance and Target Acquisition UAV. The mission capabilities of small UAVs are constantly growing by implementing recent developments in miniature computers and peripherals, electronic sensors, and optical sensing equipment at affordable cost. The requirements for the mission profile of a small UAV using the aforementioned equipment are defined with an emphasis on the potential advantages that can be offered by the nonplanar concept wing under investigation. A structural analysis using the finite element software ADINA and an aerodynamic analysis based on wind tunnel experimental data and vortex panel code results are performed. The results, compared under varying assumptions specific to an equivalent monoplane and a 
biplane, suggest potential efficiency gains for the new configuration may be possible using the nonplanar wing configuration under explicit conditions. The results also show structural characteristics and not aerodynamics alone are critical in determining the utility of this nonplanar concept. 
Dedicated to Dalila and Pietro 


\section{ACKNOWLEDGEMENTS}

I would like to acknowledge Dr. Aaron Altman, my advisor, for bringing this thesis to the conclusion with patience and expertise and for directing my steps towards a better professional and human path.

I would also like to express my appreciation to Cardinal Silvestrini for giving me the tools to complete my education and for giving me the possibility to develop my skills in a challenging environment as Villa Nazareth.

Finally my special thanks are in order to my family and my friends, who represent my restless fans and my source of inspiration. 


\section{TABLE OF CONTENTS}

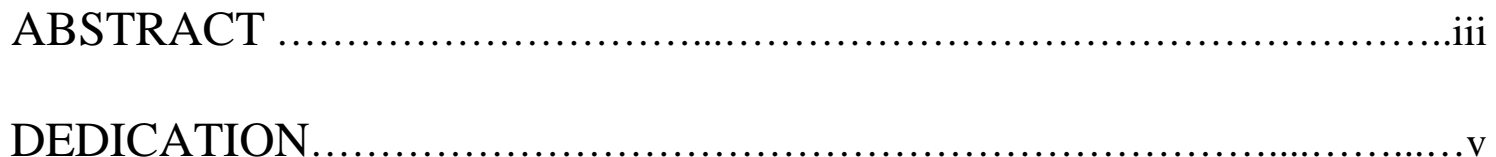

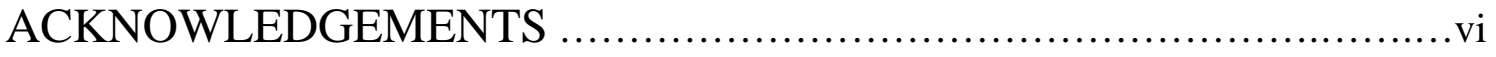

LIST OF FIGURES ......................................................

LIST OF TABLES ..............................................................

LIST OF ABBREVIATIONS AND ANNOTATIONS ........................xvi

I. INTRODUCTION ........................................................

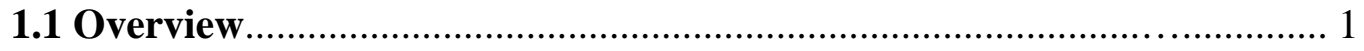

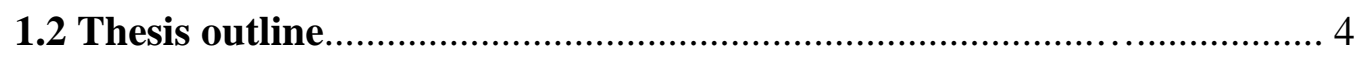

II. BACKGROUND AND LITERATURE REVIEW ................................. 6

2.1. Unmanned Air Vehicles......................................6

2.2 Nonplanar wing concepts.....................................11

2.2.1 Biplane...................................................14

2.2.1.1 Biplane:configuration and theory...................16

2.2.2 Winglets and wing tip devices..............................20

2.2.2.1 Winglet theory....................................21 
2.2.2.2 New winglet concepts.............................24

2.2.3 Closed systems............................................26

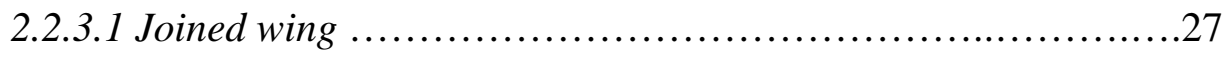

2.2.3.2 Houck configuration........................................29

2.3 Problem statement.............................................. 30

III. DESIGN REQUIREMENTS...........................................................32

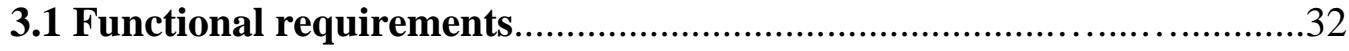

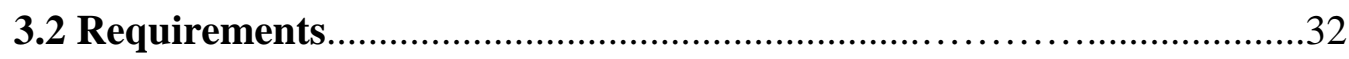

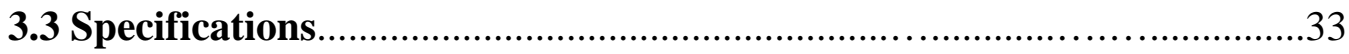

IV. AIRCRAFT INITIAL SIZING.. ...................................

4.1 The state of the Art and tabulated history ......................... 35

4.2 Constraint equations.............................................

4.3 Sensitivity analysis.........................................43

4.4 Initial Sizing. ................................................... 50

4.4.1 Payload Weight Fraction...................................50

4.4.2 Design Point.............................................51

4.4.3 Wing geometry sizing...................................53

4.4.3.1 Operating lift coefficient...........................55

4.4.3.2 Lift to drag ratio................................55

4.4.4 Propulsion.............................................56

4.4.4.1 Batteries.......................................58

4.4.4.2 Electric Motors..................................60

4.4.4.3 Selecting motor/battery combination.................62 
4.4.5 Empennage Sizing...........................................68

4.4.6 Other selected components...................................69

4.4.6.1 Autopilot..............................................69

4.4.6.2 Transmitter............................................69

4.4.6.3 Camera setup..........................................70

4.4.7 Weight Build-up............................................71

4.4.7.1 Second Iteration .....................................73

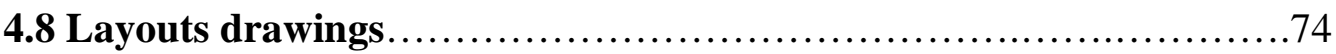

V. STRUCTURAL ANALYSIS .........................................79

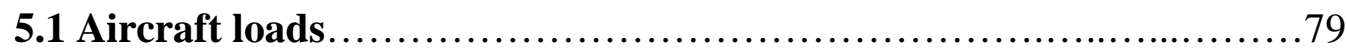

5.1.1 Maneuver loads.............................................. 79

5.1 .2 V-n diagram................................................. 81

5.1.3 Gust loads...................................................... 84

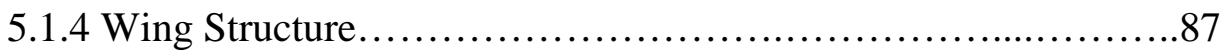

5.1.5 Load distribution.......................................... 89

5.1.6 Structural analysis........................................94

5.2 Nonplanar configuration vs standard configurations.................96

5.2.1 Methodology .............................................98

5.2 .2 Results..................................................

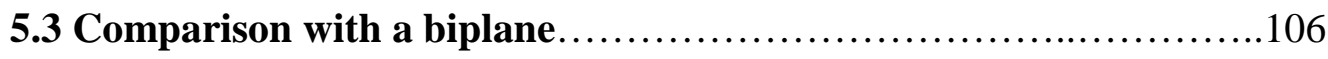

5.4 Comparison with an equivalent monoplane.........................109

VI. AERODYNAMIC ANALYSIS ........................................115

6.1 Aerodynamics of a nonplanar wing configuration....................115 
6.1.1 Results obtained using AVL software and WT Testing.

6.1.2 Aerodynamic comparison between an equivalent monoplane and the nonplanar configuration under study........................121

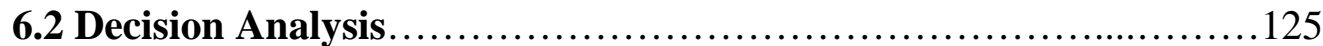

VII. CONCLUSIONS AND FUTURE WORK............................129 


\section{LIST OF FIGURES}

1. The U.S. Unmanned System Roadmap.......................................8

2. UAVS Classification....................................................... 9

3. UAVs Classification......................................................

4. Gross mass of flying object vs Reynolds Number..............................10

5. Relative vortex vs Overall Height/Span.......................................12

6. Wing geometry and trailing vortex wake.....................................

7. Gap......................................................................

8. Stagger............................................................... 18

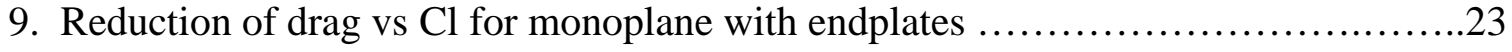

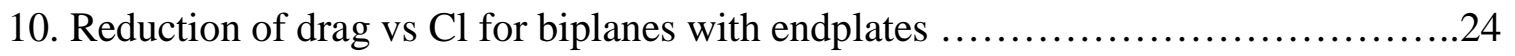

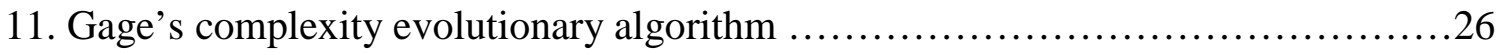

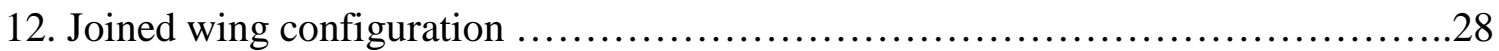

13. Houck configuration..................................................29

14. Mission profile of a Small UAVs:....................................... 37

15. Constraint diagram................................................... 42

16. Constraint diagram and previous designs...................................43

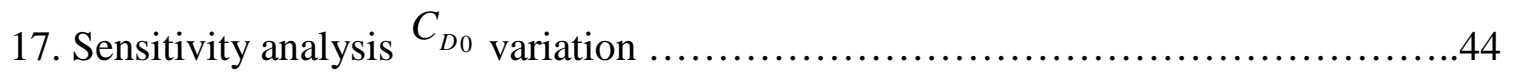




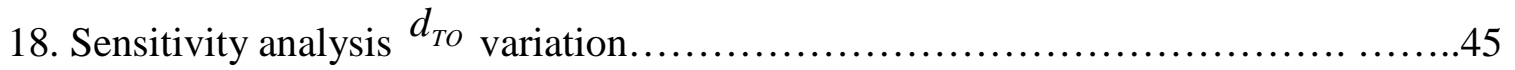

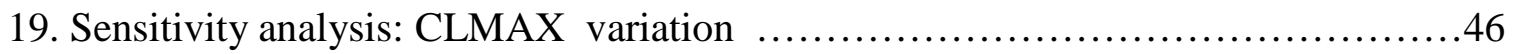

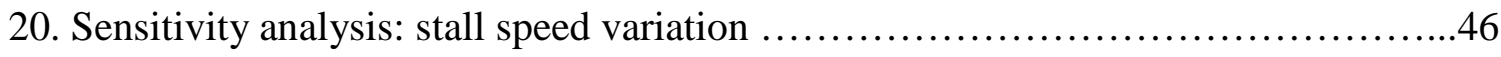

21. Sensitivity analysis: bungee cord launched aircraft ..............................48

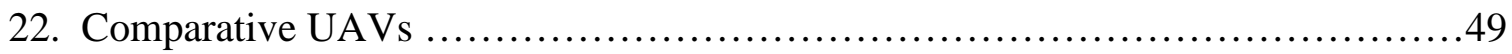

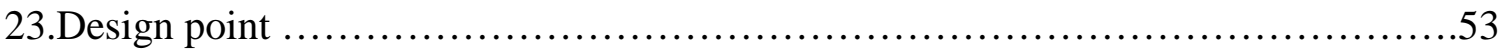

24. AR vs weight for historical airplanes........................................54

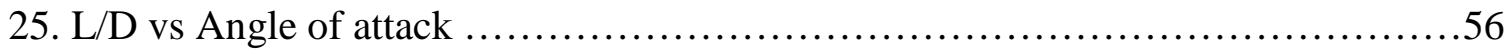

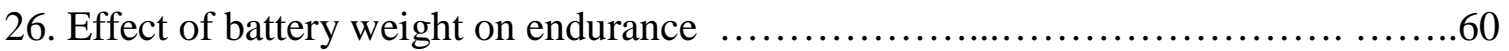

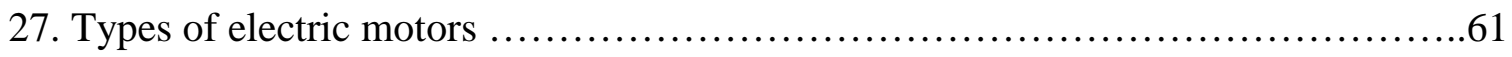

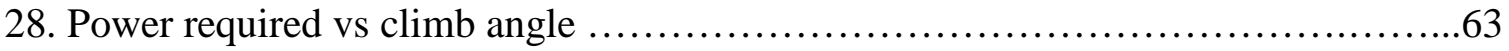

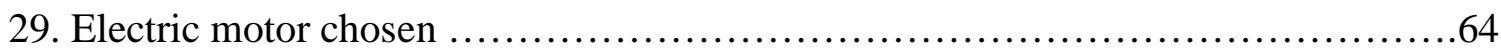

30. Minutes at cruise power vs minutes at max power..............................67

31. Camera set up and transmitter............................................... 71

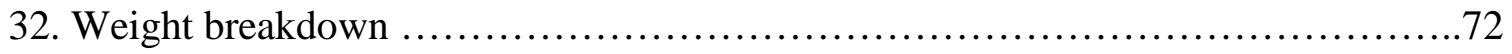

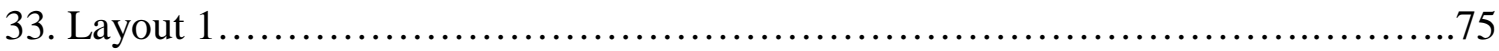

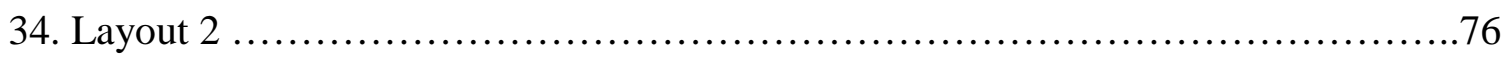

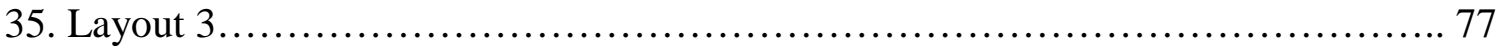

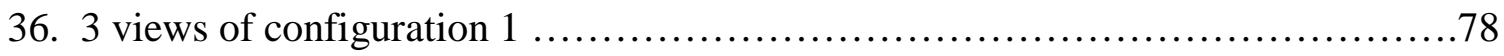

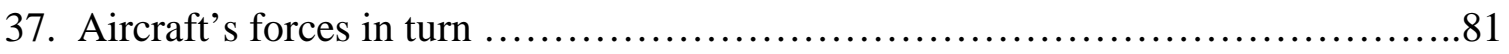

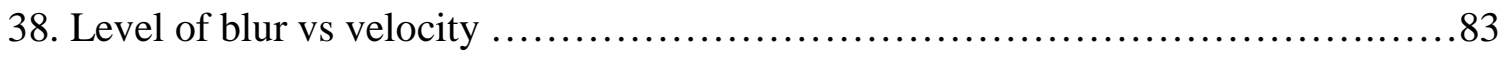

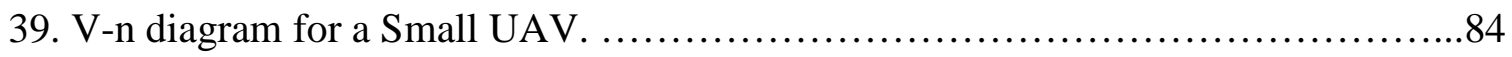

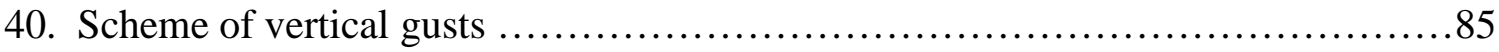




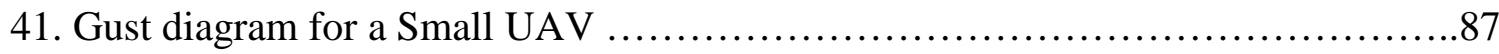

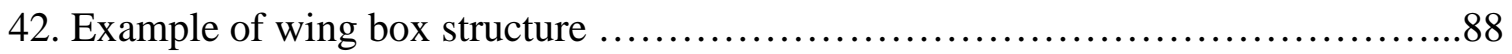

43. Elliptical distribution of the lift along the semispan ..............................90

44. Elliptical distribution vs constant-linear lift distribution ...........................91

45. Lift and drag distribution along the semi span ...............................93

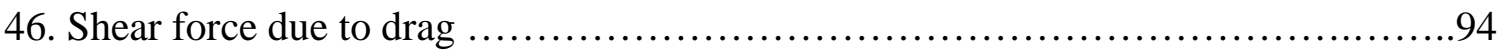

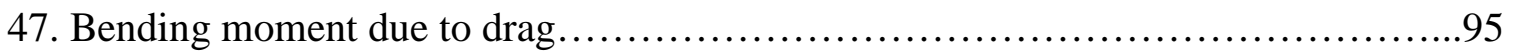

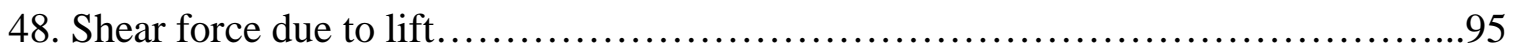

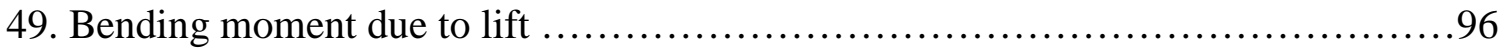

50. Finite Element Models and their geometric characteristics.......................97

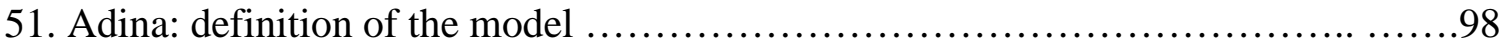

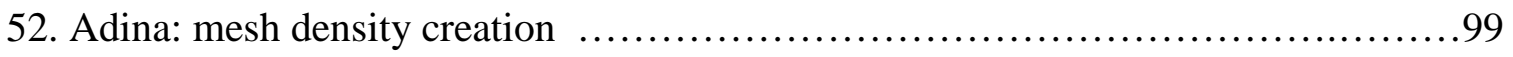

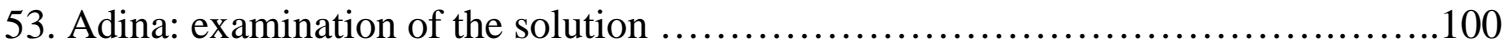

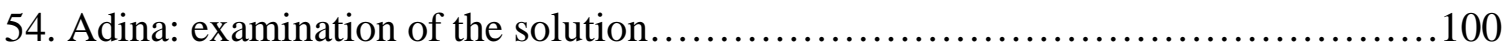

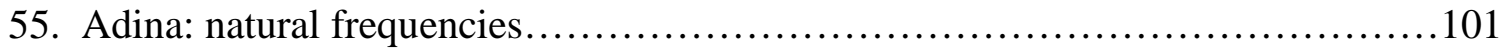

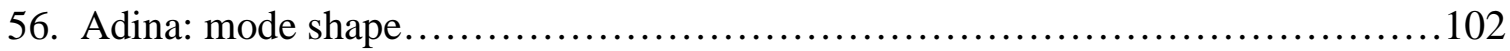

57. Adina: configuration with the smallest displacement............................104

58. Adina: configuration with largest displacement ................................104

59. Adina stress distribution of configuration with largest displacement and effective

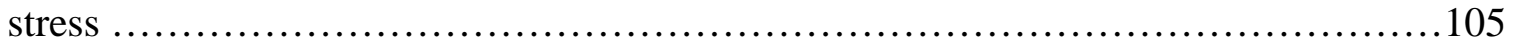

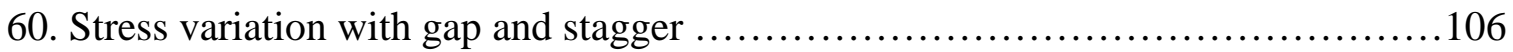

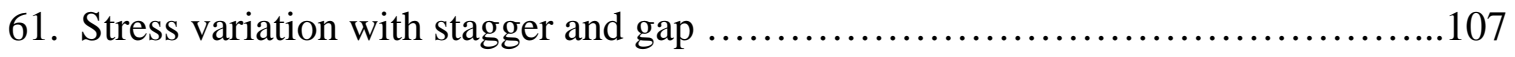


62. Adina: stress distribution of an equivalent biplane with 0 stagger and 1 c gap .108

63. Adina: Effective stress distribution of an equivalent monoplane 110

64. Adina: Stress distribution of an equivalent monoplane with same span of the

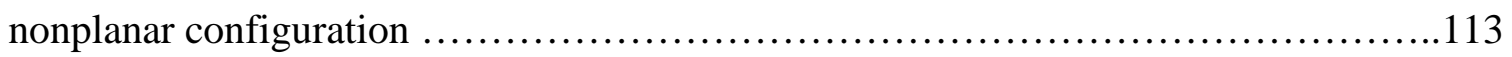

65. Stagger vs lift....................................................... 116

66. Gap vs lift..........................................................117

67. AVL lift vs angle of attack .............................................. 118

68. Experimental results L/D vs angle of attack for different stagger configuration 120

69. Experimental results L/D vs angle of attack for different gap configurations... .....120

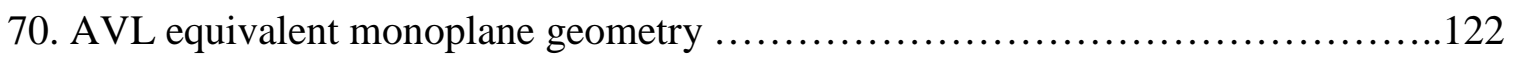

71. $\mathrm{Cl}$ vs angle of attack: monoplane vs biplane.............................123

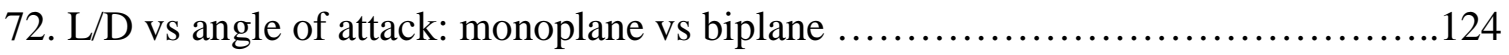




\section{LIST OF TABLES}

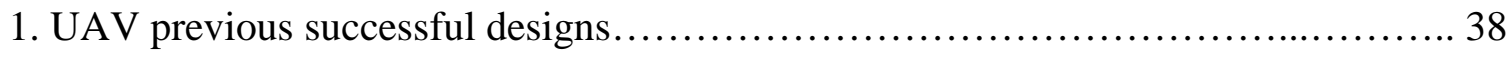

2. Historical values............................................................... 50

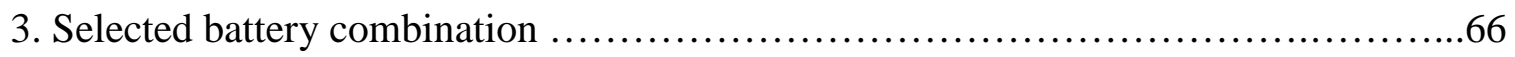

4. Vertical and horizontal tail specifications.................................68

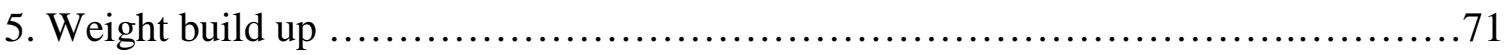

6. Design parameters.........................................................

7. UD for three characteristic conditions: gust, cruise and dive $\ldots \ldots \ldots \ldots \ldots \ldots \ldots \ldots . \ldots 6$

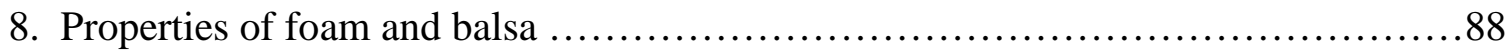

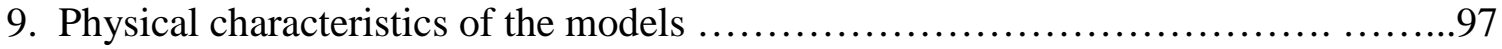

10. Displacement and maximum effective stress................................103

11. Displacement and maximum effective stresses for a biplane...................107

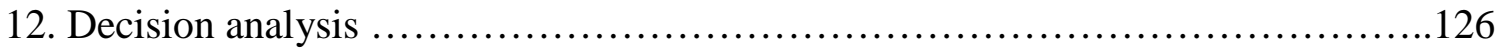




\section{LIST OF ABBREVIATIONS AND ANNOTATIONS}

\begin{tabular}{|c|c|c|c|}
\hline$A R$ & aspect ratio & $\mathrm{L}$ & lift force \\
\hline$b$ & span & $\mathrm{P}$ & Power \\
\hline$C$ & chord & $R e$ & Reynolds number \\
\hline $\mathrm{C}$ & Capacity & $s$ & stagger \\
\hline $\mathrm{C}_{\mathrm{D}}$ & drag coefficient & $\mathrm{S}$ & reference surface \\
\hline $\mathrm{C}_{\mathrm{Do}}$ & parasite drag & $\mathrm{t}$ & thickness \\
\hline $\mathrm{C}_{\mathrm{Di}}$ & induced drag & $\mathrm{T}$ & thrust \\
\hline$C_{L}$ & lift coefficient & $V_{p}$ & potential difference \\
\hline$C_{\operatorname{Lmx}}$ & max lift coefficient & $\mathrm{V}_{\text {stall }}$ & stall velocity \\
\hline $\mathrm{D}$ & drag force & $\mathrm{W}$ & weight \\
\hline $\mathrm{E}$ & Young's Modulus & $v$ & Poisson's Ratio \\
\hline$g$ & gap & $\Delta \sigma$ & var. in max. eff. stress \\
\hline I & current & $\rho$ & density \\
\hline
\end{tabular}




\section{Chapter I}

\section{Introduction}

\subsection{Overview}

Looking at the flying characteristics of insects and soaring birds, biologists and then aerodynamicists found some of them used multiple lifting surfaces, as biplanes, winglets or pin feathers, to increase their flight efficiency. Several studies were subsequentlly performed in order to investigate the potential of using multiple lifting surface concepts for reducing drag. The interest in wingtip devices as dragreduction devices spans the last 25 years. This research focuses on an effort to maximize the structural and aerodynamic efficiency of a new multiple lifting configuration similar to the Houck Configuration. The Houck Configuration consists of an upper and lower set of wings joined at the two wingtips by curved flow guides. The Houck patent claims to offer the possibility of reducing drag and therefore improving the general performance of an aircraft. The configuration analyzed in this research, with respect to the Houck Configuration, has flat endplates and not curved flow guides. More specifically the aim is to determine if this particular nonplanar wing concept can meet the requirements of the mission of a small Reconnaissance, Surveillance and Target Acquisition UAV. In response to the requirements of this mission profile, the conceptual design of a small non planar wing UAV is completed for this thesis.

This study, therefore, addresses this domain, the domain of Conceptual Design of small UAVs, looking, in particular, at the possibility of taking aerodynamic and 
structural advantage by selecting this new multiple lifting surface configuration in comparison to a standard monoplane or biplane wing.

The conceptual design process of an aircraft is an act of creativity, and like creative endeavours, there isn't a unique point where the process begins. Design is an iterative effort and, by its very nature, multidisciplinary, requiring input from a variety of disciplines. To quote Stinton [1] - it is "a mixture of precise science, disciplined methods, and consummate accuracy in telling it as it is after a test flight, gut feeling and artistry”. These various strands combine into a deluge of data and one of the great challenges lies in handling and distilling this.

These concepts apply to fixed wing aircraft design in general, but some of the challenges of Unmanned Air Vehicle (UAV) conceptual design differ slightly from those encountered by the designers of manned aircraft. UAV airframe design usually involves considerably more topological freedom for two main reasons. First, the UAV world has not yet reached the maturity that characterizes the design of, say, commercial airplanes, whose shape and topology is the result of a vast amount of commercial pressure and design effort over a century of passenger air transport. Observing existing commercial aircraft designs has provided useful empirical relationships and insights for the designer to apply yielding a relative well defined problem. Secondly, engineering a UAV is a less constrained process than producing a new manned aircraft concept. The reasons for this range from having fewer certification criteria to not having to shape the fuselage to accommodate the crew and/or passengers in a comfortable fashion. And therein lies the difficulty. With all the advantages of this freedom comes a design space that is far larger than that normally considered by, say, the designers of passenger airliners and it is therefore more diffcult to explore in the course of the conceptual design process. This also 
results in a lack of a historical statistical database of the main design parameters of UAVs, useful for the first steps of the design of an aircraft.

This new challenge for aircraft designers is occurring because UAVs are constantly drawing more and more attention in various sectors of military and civil applications. Grasping the recent developments in miniature-sized computers, their peripherals, electronic sensors, and optical sensing equipment at affordable cost, the mission capabilities of small UAV's are ever increasing.

In particular, in the mid-1990s the U.S. Army realized that there was a need for a small UAV that could be used for Reconnaissance, Surveillance, and Target Acquisition at the company and platoon level. As years passed, the need for small UAVs continued to increase with forces deploying to engage in asymmetrical warfare. Small reconnaissance planes can loiter about a ground target over a hill or behind a building, taking photos and live video and sending visual real-time information to the ground station without being detected and without exposing human personnel. With their small size and light weight they can easily be transported where the surveillance is needed. For all these reasons small UAVs have been compared to a pair of long-distance binoculars that can see behind hills. The beauty of this class of aircraft lies in its ability "to go out there and to reach out past us, to find the threat before it even gets closer ... before our troops get to harm's way” [2]

Companies including Northrop Grumman, Boeing, and AeroVironment are among the major developers of SUAV's currently used for military purposes. Fully autonomous small UAVs now on the market range in takeoff methods from the Raven (by AeroVironment), which is hand-launched to the Lockheed Martin Desert Hawk, which is launched with a bungee chord. Under development are also a lot of Micro 
UAVs, called MAVs, which have a maximum dimension and weight on the order of $15 \mathrm{~cm}$ of span and 150 grams, respectively.

From an aerodynamic point of view a potential gain in endurance by reducing drag or, from a structural point of view, a reduction in the weight of the wing, would translate to a lighter aircraft with an increased endurance and a reduced stall speed that, in the end, are three of the main keys, as will be specified in the chapter 3 , for the mission profile of this class of aircraft.

\subsection{Thesis outline}

After a birds-eye view of the problem and the reasons why it is worthwhile to investigate, the next chapter gives background information necessary to understand a few of the fundamental concepts that will be the basis of the discussion throughout the thesis and a review of the major ideas in the state of the art of Small UAV and non planar wing concepts.

Then at the end of the next section, a coincise statement of the problem that this thesis addresses is provided with justification, by direct reference to the state of the art, what this research can add to the knowledge of nonplanar wing concept designs and why it is worthwhile to investigate.

The following chapters try to answer the questions of the thesis. The design requirements for the mission profile of the proposed UAV aircraft are defined, and the conceptual design of an aircraft is completed. A structural analysis of the configuration under study is performed using the finite element software Adina and compared, in different ways, to a standard monoplane configuration. These results are then incorporated into an aerodynamic analysis based on low speed wind tunnel experimental data and panel code results. 
In the last chapter the main inferences, on the basis of the study done, are reported with a summary of the contributions of new knowledge that this thesis makes and a list of useful suggestions for potential future research. 


\section{Chapter II}

\section{Background and Literature Review}

\subsection{Unmanned Air Vehicles}

According to Newcome [3] in his Unmanned Aviation: A Brief History of Unmanned Air Vehicles, for more than eight decades UAVs have been referred to as robotic airplanes, remotely piloted vehicles (RPVs), aerial torpedoes, and drones. The term RPV was replaced by UAV only in the 1990s, and now the U.S. Defence Department dictionary defines a UAV in the following way:

A powered, air vehicle that does not carry a human operator, uses aerodynamic forces to provide vehicle lift, can fly autonomously or be piloted remotely, can be expendable or recoverable, and can carry a lethal or nonlethal payload. Ballistic or semi ballistic vehicles, cruise missiles, and artillery projectiles, however, are not considered unmanned vehicles [4].

Interest in unmanned air vehicles was stimulated by World War I and led to full-sized airplanes with primitive controls capable of stabilizing and navigating without a pilot onboard. The conversion of manned airplanes to target drones 
continued during the 1920s and 1930s. During World War II a large number of radiocontrolled, radar-controlled, and television controlled glide bombs were proposed and used. The most famous were the U.S. Navy TDR-1, the U.S. Army AZON, the German Fritz-X.

The availability of small internal combustion engines and small radio receivers and trasmitters plus the invention of control surface actuators in the 1930s led to the era of radio controlled model airplanes. Continuous improvements in RC model equipment, including the introduction of electric motors, plus advances in micromechanical systems and microelectronic components and sensors led to the feasibility of small air vehicles in the 1990s and since that moment the interest in the design and the development of small unmanned air vehicles has increased dramatically until the present.

This interest in UAVs and Small UAVs is even supposed to increase in the next 25 years. The US Unmanned Systems Roadmap 2007 - 2032 [5] predicts, in fact, a general development and employment of an increasingly sophisticated force of unmanned systems, as it is shown in Fig. 1 where UAS means Unmanned Aircraft System, UGV Unmanned Ground Vehicle, UUV unmanned underwater vehicle and USV unmanned surface vehicles. 


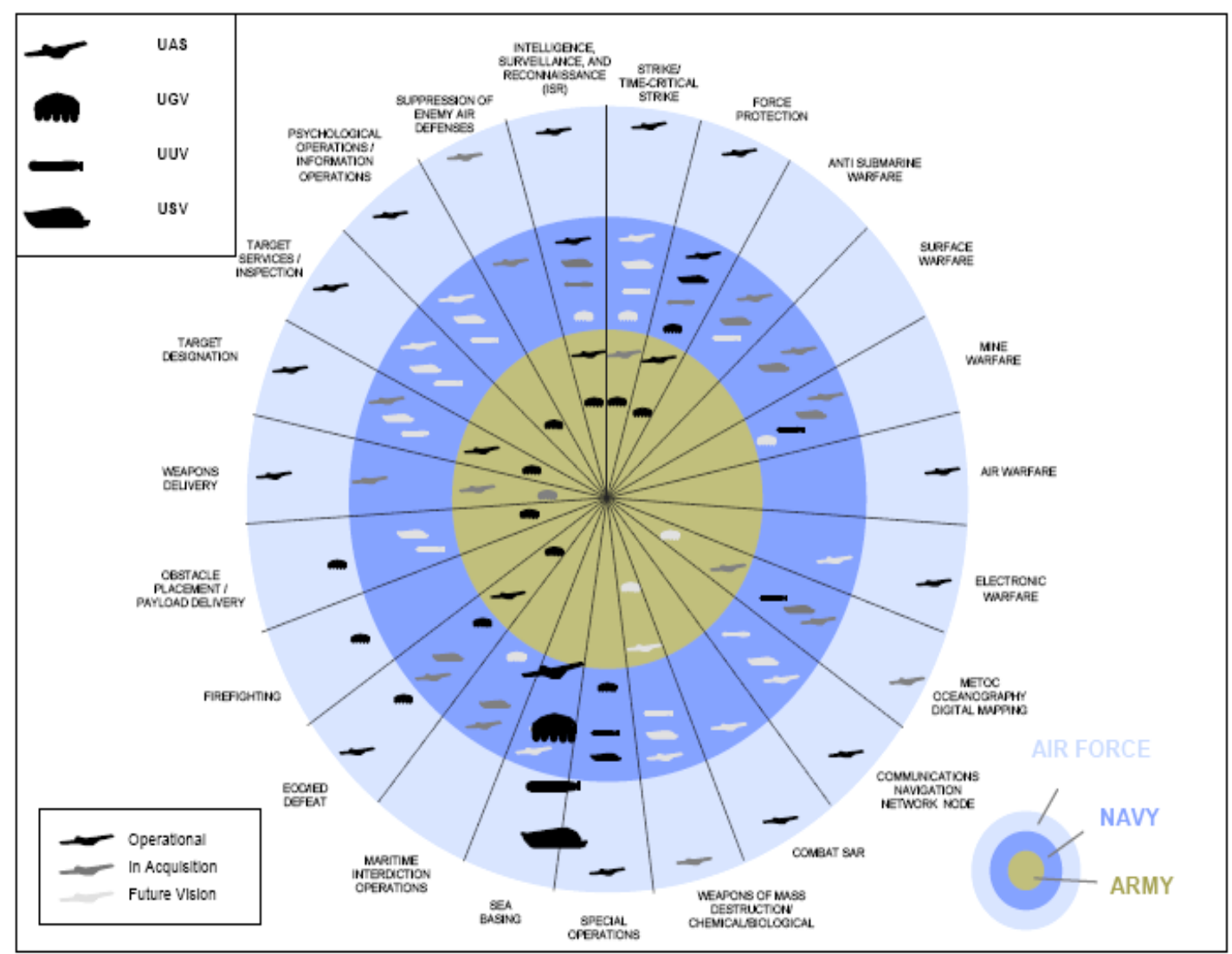

Fig. 1 (reproduced from [5]): The U.S. Unmanned System Roadmap. US Unmanned System Roadmap 2007-2032 predicts a general development and employment of unmanned systems in the future. In particular, the figure shows an increase in the number of UAS for Oceanography digital mapping and suppression of enemy air defences by the Air Force and an increase in UAS for communications, navigation network node, electronic warfare, psychological operations by the Navy.

One of the possible classifications of UAVs is with respect to the size and weight. In this case UAVs can be divided into four different groups: Micro, Small, Medium and large UAVs as shown in Fig. 2. The JUAS COE (Joint Unmanned Aircraft System Center of Excellence) has divided UAVs, on the basis of airspeed, weight and operating altitude, into 5 categories as shown in Fig.3. 


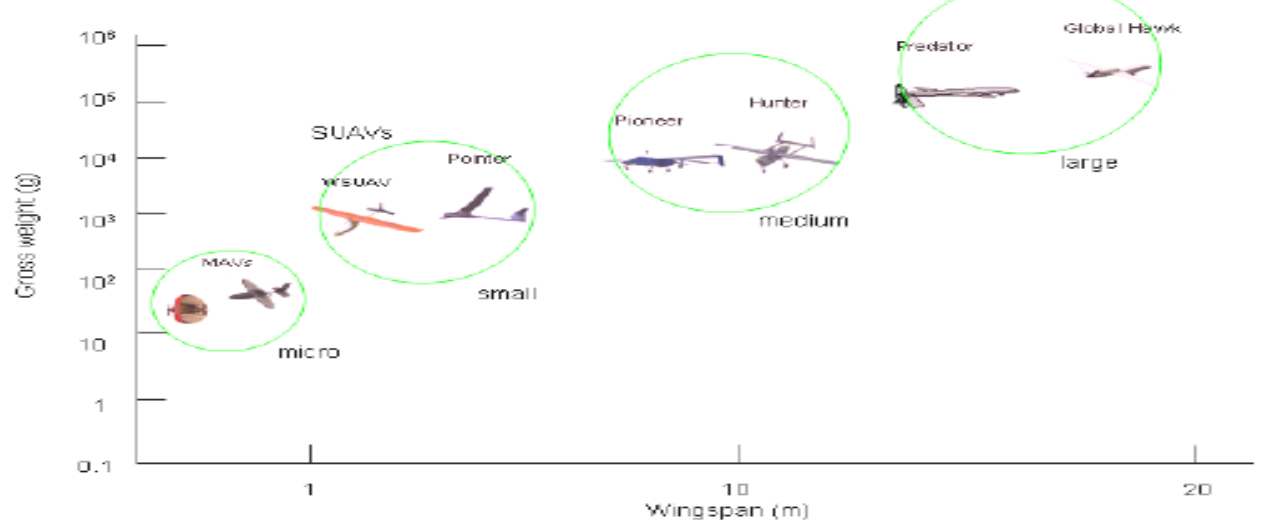

Fig. 2: UAVS Classification. UAVs can be classified in different ways. This figure represents a UAV classification with respect to weight and wingspan. In this case there are 4 different groups: micro, small, medium and large. This thesis focuses on small UAVs.

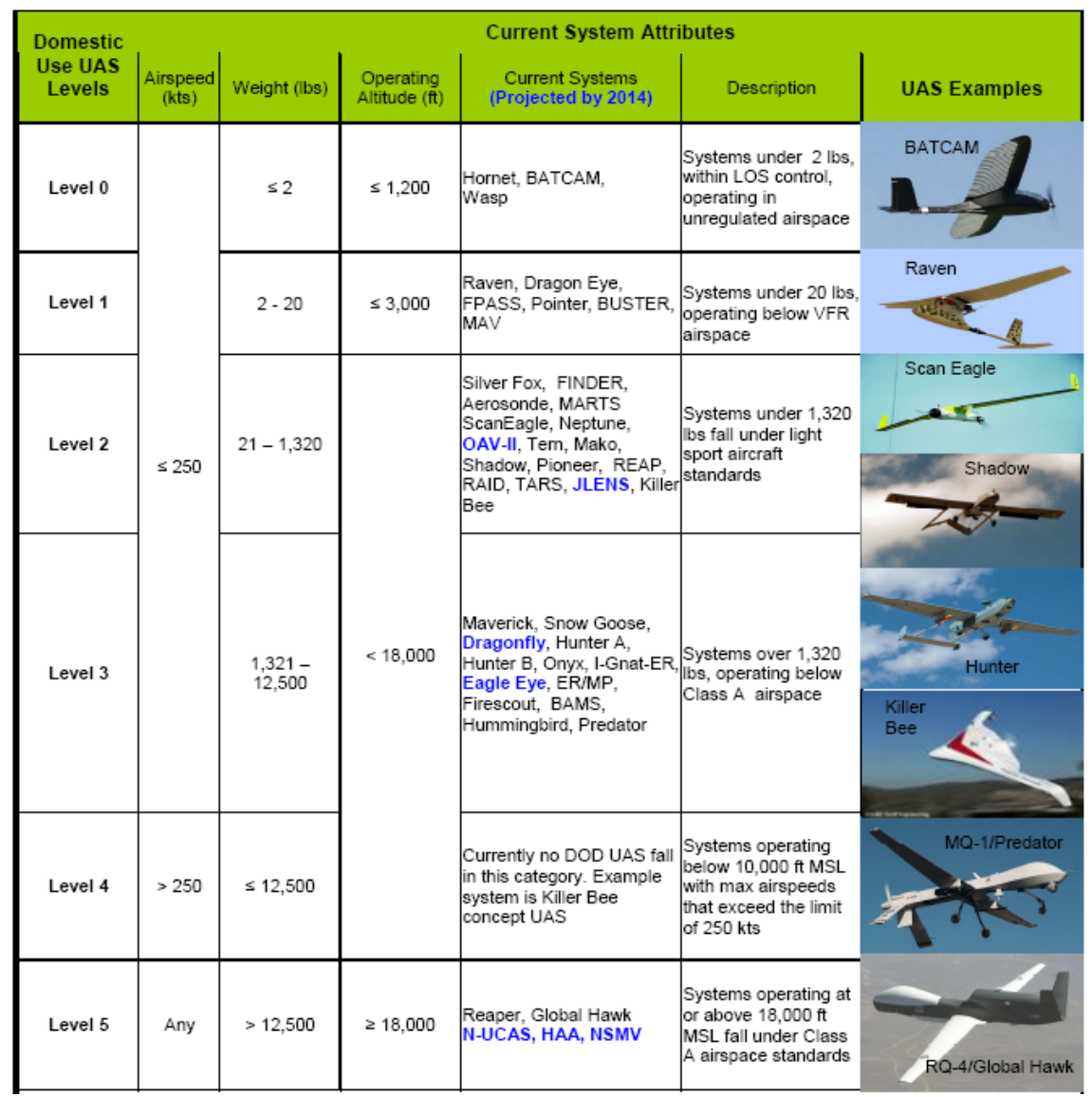

Fig. 3 UAVs Classification (reproduced from [5]): UAVs can be classified in different ways. JUAS COE gives a classification on the basis of airspeed, weight and operating altitude. This thesis focuses on level 1 UAS. The weight ranges form 2 to $20 \mathrm{lbs}$ and the operating altitude is less than 3,000 feet. 
This research focuses on the design of a Level 1 Unmanned Air System, referred to as a Small UAV. The state of the art of this particular class of aircraft is reported in detail in chapter 4 . The gross mass of small air vehicles and other flying objects vs chord Reynolds number is shown in Fig. 4.

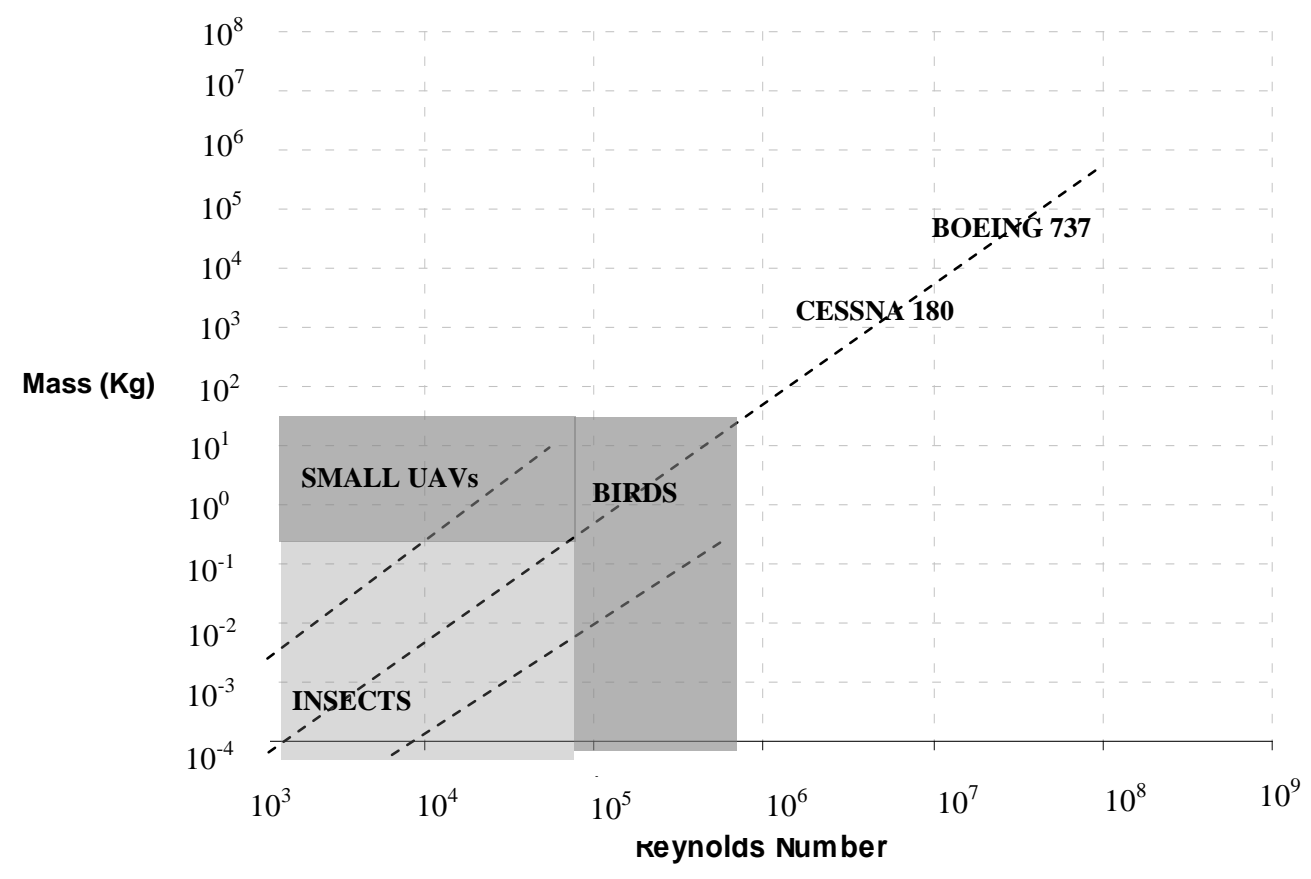

Fig. 4 Gross mass of flying object vs Reynolds Number (partly reproduced from [6]): The graph shows the gross mass of small air vehicles and other flying objects vs chord Reynolds Number The Reynolds Number of Small Uavs has a similar order of magnitude as many birds as well as similar weight which may imply also similar aerodynamic behaviour.

It's interesting to note that, as presented in Fig. 4, the Reynolds Number of Small UAVs have a similar order of magnitude as many birds and as well as having similar weights. 


\subsection{Nonplanar wing concepts}

Nonplanar wings include configurations such as biplanes, ring-wings, joined wings, and wings with winglets. Such designs are of interest because of their potential to lower vortex drag without increased span, a key constraint for many aircraft. The vortex drag accounts for a large fraction of airplane cruise drag and therefore any concept that results in reduction of vortex drag may potentially have a significant effect on fuel consumption, endurance and range characteristics. Induced drag can be also reduced by increasing the span of a planar wing at constant chord. But one of the reasons wingspans are not increased to reduce drag is that the higher structural weight and cost make such efforts counterproductive. Therefore, as it can be seen even in first considerations, nonplanar wing concepts must be assessed taking into account not only the potential improvements in aerodynamic features, but also their structural/weight characteristics. In fact some design concepts are promising more because of their structural characteristics than their aerodynamic features. For example some nonplanar concepts utilize the differences between nonplanar and planar wing load variation with lift coefficient to reduce structural loads under critical conditions and therefore save weight or add span at fixed weight. Sometimes other non-aerodynamic features are of interest as well including effects on stability and control and characteristics of wake vortices.

Before going into the details, it's worth an evaluation of the magnitude of the potential gains associated with nonplanar wings. To this aim the results obtained by Ilan Kroo [3], will be analyzed. They were computed using an optimizing vortex lattice code but agree with classical solutions from Prandtl, von Karman and Burgers, Cone, and Jones. The minimum vortex drag for systems with the same geometric span 
and carrying the same total lift is shown in Fig. 5 for biplanes, boxplanes, a ring-wing, and winglets with varying ratios of height to span. They are all compared to a monoplane with the same span and lift.

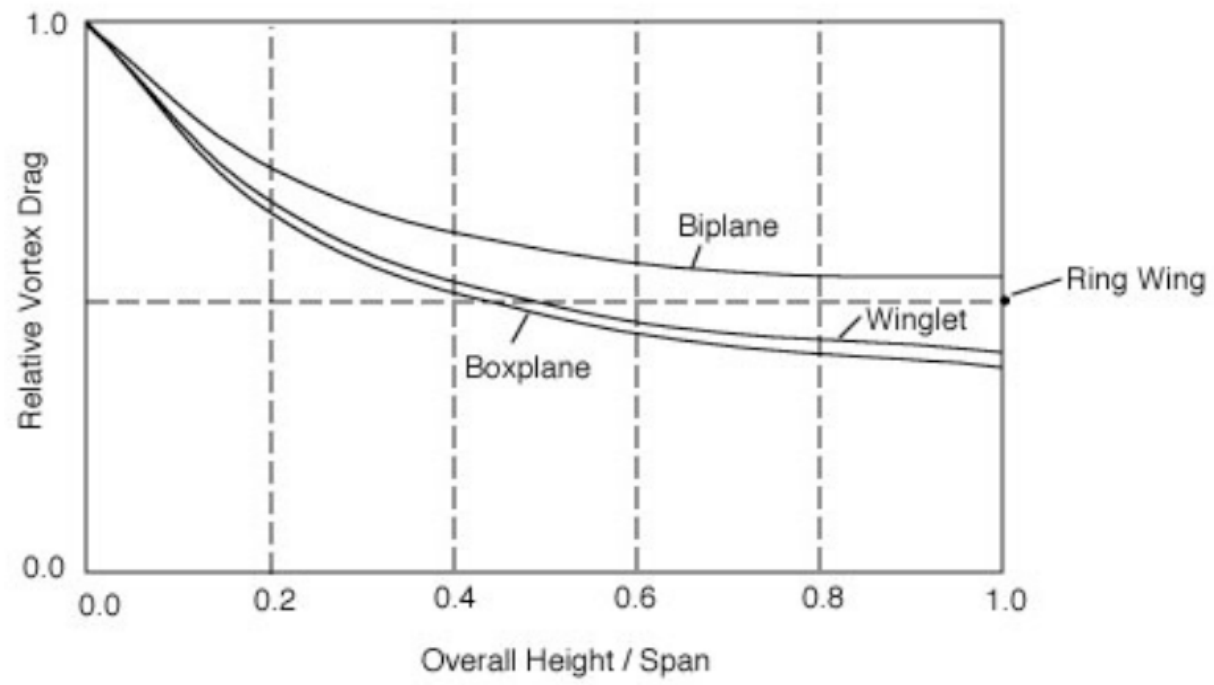

Fig. 5 Relative vortex vs Overall Height/Span (partly reproduced from [6]): From the results obtained by Kroo [6] a ring wing has half of the vortex drag of a monoplane of the same span and lift, and the boxplane has the lowest drag for a given span and height. From an aerodynamic point of view it means that keeping fixed the span considerable savings in induced drag can be achieved if large vertical extents are permitted. Of course structural considerations must be considered too.

Fig.5 shows that a ring wing has half of the vortex drag of a monoplane of the same span and lift, a biplane achieves this same drag savings in the limit of very large gap and the boxplane has the lowest drag for a given span and height although winglets achieve similar aerodynamic savings. In other words, according to Kroo, considerable savings in induced drag can be achieved for a fixed span if large vertical extents are permitted. On the other side, savings in induced drag on the order of $30 \%$ are possible with a height to span ratio of 0.2. Another encouraging result is shown in Fig. 6 [6], where the span efficiency for several nonplanar geometries of the same projected span and lift (with a vertical extent of $20 \%$ of the wing span) is illustrated. 

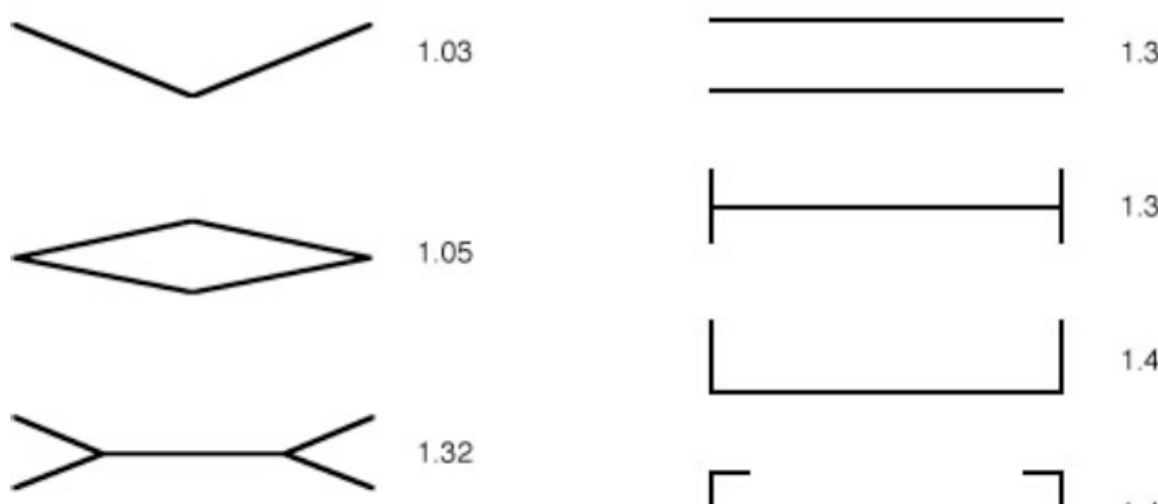

1.32

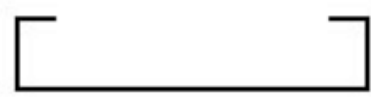

1.45

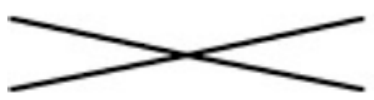

1.33

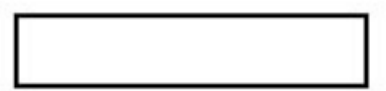

1.46

Fig. 6 wing geometry and trailing vortex wake: Specifying the geometry of the trailing vortex wake and solving for the circulation distribution with minimum drag, Kroo obtained as a result that the boxplane represents the minimum solution. All the non planar geometries he took in consideration have same projected span and lift.

These results were obtained by Kroo by specifying the geometry of the trailing vortex wake and solving for the circulation distribution with minimum drag. Therefore Fig.6 must be interpreted by keeping in mind the assumption that they are optimally twisted. The results show the vertical extents of the system near the tips is the critical parameter and that the boxplane represents the minimum solution.

Alternatively it is also very important to analyze the disadvantages these configurations have. First, as purposely fully emphasized, these results offer a potential lower vortex drag under the assumption of fixed span. This means that if this constraint fails, these results are not reliable anymore. Besides, adding vertical surfaces as winglets adds wetted area. Profile or form drag is related to the size of the aircraft whereby the winglets would increase size. Skin friction is related to the friction of the fluid flow over the surface area of the aircraft whereby flow guides would increase surface area. Interference drag is caused the interference of boundary 
layers caused by other portions of the aircraft whereby the flow guides would increase interference. If the reduction of induced drag is greater than the increase in parasite drag (profile drag, skin friction drag, and interference drag) then the total drag will be reduced and then these designs can be applied to various applications where aerodynamic efficiency at higher lift is desired.

The results of this research agree with the point of view of Kroo, confirming, in later chapters, the importance of structural characteristics in determining the utility of nonplanar concepts for a potential efficiency gain.

After this general discussion about nonplanar concepts the next section reviews some specific concepts of the past, their advantages and disadvantages and some important theoretical concepts associated with them that will be revised in later chapters.

\subsubsection{Biplane}

The simplest way to create a nonplanar configuration is to use just 2 noncoplanar wings, that is the biplane. With the beginning of powered flight, so came the concept of the biplane. In 1903 the Wright brothers found the high lift and structural rigidity of the biplane to be the answer to putting man in the air. Based on their own tests and those of Otto Lilienthal, it was apparent that at very low Reynolds numbers highly cambered thin sections performed much better than thicker sections. Because of the low flight speeds required for take-offs and landing and for the power plants available to the Wrights, the design needed to be light and incorporate large wing areas. This requirement was well met by the biplane configuration. The development and improvements of the biplane continued from that moment on. Biplanes dominated aviation for the first 30 years. The designers of early aviation, in fact, used this 
configuration mainly because of the large engine weight, which required a large wing area that could be accounted for without increasing the wing span. Furthermore the structural integrity of the biplane configuration was much higher than the monoplane due to the wing trusses, giving rise to fewer structural failures. Besides, at the low speeds being flown, a high degree of maneuverability was realized with this configuration due to an increased roll rate. As structural materials and technology improved, thicker airfoils and longer wing spans were made possible therefore designers made the decision, due also to other factors such as engine weight reduction and higher flight speeds, to switch from a biplane to a monoplane and now the biplane arrangement is mainly reserved for recreational purposes.

Over the past 20 years, however, a renewed interest in drag reduction has stimulated new conceptual designs incorporating the biplane configuration. In fact theoretically the induced drag of a biplane should be lower than that of a monoplane of equal span and total lift because the nonplanar system [6] “...can influence a larger mass of air, imparting to this mass a lower average velocity change, and therefore less energy and drag..”. A biplane, if the 2 wings are separated vertically by a very large distance, produces exactly half of the induced drag of a monoplane with same span. Induced drag is proportional to the square of the lift being generated. If that lift is split evenly between the 2 wings, then each wing should have only one-fourth of the drag of the original wing [7]. Therefore the total induced drag for the biplane system should be one half of the induced drag of a monoplane with same span. But mutual interference effects prevent taking advantage of the full reduction and so a good design can yield on the order of a 30\% reduction [7] in induced drag. The high parasite drag of struts and cable bracing substantially reduce these savings. Now that cantilever structures can be built efficiently one might ask if a modern biplane can 
provide some reduction opportunities. This was suggested by Lange (1974) and designs such as Rutan’s Quickie (Downie 1984) have achieved some success. But practical considerations such as Reynolds number effects upon airfoil drag, aeroelastic problems, fuel volume and structural weight usually overwhelm potential vortex drag advantages.

The main important concepts of the biplane configuration and of biplane theory will be presented next in order to ease the transition to the next chapters of the thesis.

\subsubsection{Biplane: configuration and theory.}

Two years after Prandtl published his famous papers on the theory of lift, Munk published General Biplane Theory [8], incorporating Prandtl's ideas and his own on the interaction of two lifting surfaces. He identified five key geometrical variables in the analysis of the biplane that are used also in the next chapters:

- Gap

- Stagger

- Aspect Ratio

- Decalage

- Chord

Later research has been done on the effect of sweep, dihedral, overhang and winglets on the aerodynamic efficiency of the biplane.

Aspect ratio [8] is the ratio of the square of the maximum span b to the total area $\mathrm{S}$ of a particular wing platform. On a biplane, there may be a different aspect ratio for each respective wing. 
Chord - Datum line joining the leading and trailing edges of the airfoil, and taken to be the mean geometric chord if there is a taper ratio.

Gap (shown in Fig. 7) - The distance between the planes of the chords measured along a line perpendicular to the chord.

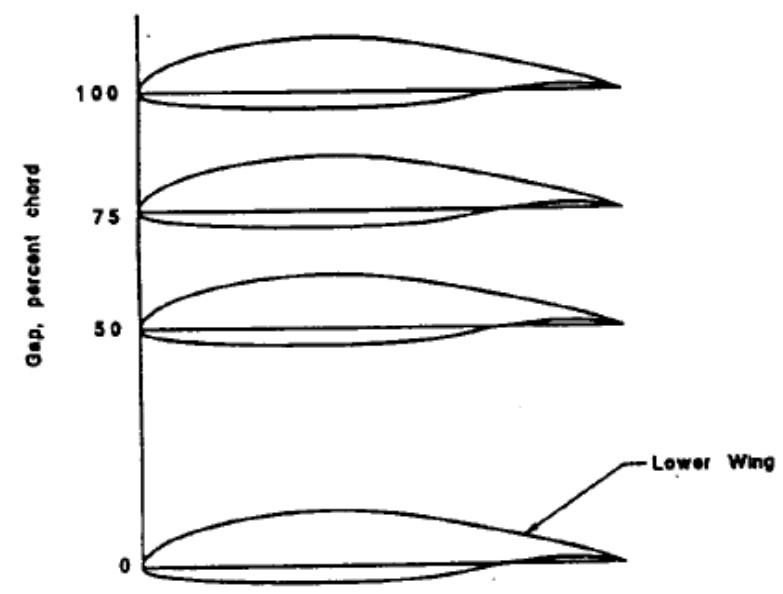

Fig. 7 Gap (reproduced by [8]): The gap of a Biplane is one of the main geometrical characteristics of the biplane configuration. It is the distance between the planes of the chords measured along a line perpendicular to the chords

Stagger (shown in Fig. 8) - the longitudinal offset of the two wings relative to each other. It is considered positive when the upper wing is forward and is measured from the leading edge of the upper wing along its chord to the point of intersection of this chord with a line drawn upward and perpendicular to the chord of the upper wing at the leading edge of the lower wing. 


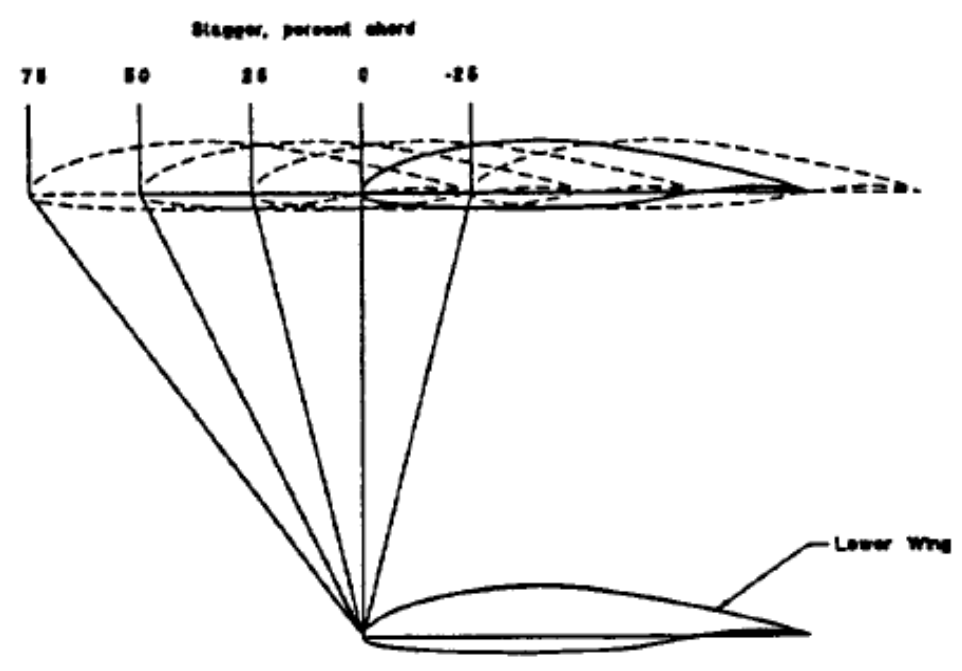

Fig. 8 Stagger (reproduced by [8]): The stagger of a Biplane is one of the main geometrical characteristics of the biplane configuration. It is the relative longitudinal position of the wings on a biplane. Positive Stagger is when the upper wing's leading edge is in advance of that of the lower wing and vice versa for Negative Stagger

Decalage - The acute angle between the wing chords of a biplane usually considered positive when the lower wing is at a lower incidence angle than the upper wing.

Sweepback - A wing design in which the leading edge (and sometimes the trailing edge) slope in planform is such that the wing tips are further, forward or aft, than the wing root.

Dihedral Angle - The acute angle between the horizontal plane and the plane of the chords of the wing.

Overhang - The ratio of the difference in span of the upper wing to the lower wing of a biplane.

The drag of an aircraft can be broken down into two main parts: Parasite drag, and induced drag. Because of the biplane lift interference effects, Munk studied the induced drag of a biplane due to the interference of one wing on the other. These 
studies led to the following main theoretical results, under the assumption that each wing was elliptically loaded:

I. Munk's stagger theorem, which states that total induced drag of any multiplane lifting system is unaltered if any of the lifting elements are moved in the direction of the motion provided that the attitude of the elements is adjusted to maintain the same distribution of lift among them.

II. The theoretical expression for determining the induced drag coefficient of a biplane compared to that of a monoplane, where $C_{D 1}$ and $\mathrm{C}_{\mathrm{L}}$ are the induced drag and lift coefficients of the monoplane respectively:

$$
C_{D 2}=C_{D 1}-\frac{C_{L}^{2}}{\pi}\left[\left(\frac{S_{1}}{b_{1}^{2} k_{1}^{2}}\right)-\left(\frac{S_{2}}{b_{2}^{2} k_{2}^{2}}\right)\right]
$$

Where the interference factor

$$
k^{2}=1.8\left(\frac{G}{b}+1\right)
$$

And for the monoplane is equal to 1 .

Prandtl then reaffirmed Munk's stagger theorem by stating that the sum of the induced downwash between the two wings will remain constant, given any longitudunal change in geometry and at angles of attack such that the lift is constant. He also derived a theoretical expression for determining the induced drag similar to that of Munk:

$$
D_{i}=\frac{L_{1}{ }^{2}}{q \pi b_{1}^{2}}+2 \frac{L_{1} L_{2} \sigma}{q \pi b_{1} b_{2}}+\frac{L_{2}^{2}}{q \pi b_{2}^{2}}
$$

where the interference factor,

$$
\sigma=\frac{1-0.66 G / b}{1.05+3.7 G / b}
$$


Was computed by integrating the downwash associated with the larger wing over the span of the smaller wing's wake in the Trefftz plane and so was a function only of the span ratio and the vertical gap between the wings.

From the theoretical results of Munk and Prandtl, if the two wings had the same span and carried the same lift, the induced drag for a biplane is smaller than that of a monoplane with the same total lift and span.

Later these theories were refined. Clark B. Millikan adopted a theory from Dr. Theodore Von Karmàn known as Airfoil Theory. In his paper, Millikan presented and used Von Karmàn's theory to develop a procedure for determining the characteristics of the individual wings of an arbitrary biplane configuration without sweepback or dihedral.

Although this process showed great success over current theories when compared with experimental data, the procedure was very tedious and therefore was rarely incorporated into use by the designers of that day. Walter Diehl [9], then, published 2 more practical reports on biplane theory in which he combined experimental and theoretical data by Fuchs and Hopf [10] to obtain a series of curves from which the lift curves of the individual wings could be found.

\subsubsection{Winglets and wing tip devices}

Due to the concentration of vorticity near the wing tip, devices to redistribute and interact with the vorticity in this region have been studied since the introduction of finite wing theory. Although low aspect ratio end plates were originally thought to retard the formation of tip vortices, the operation of such devices is now more commonly understood through the interaction with wake vorticity. Small tip devices are not able to eliminate or diffuse the vortex wake but the reduction of the induced 
drag is related, as for span extension, to the additional bending moment added to the wing. To produce a large change in the vortex drag without a large increase in wetted area, low aspect ratio endplates were replaced by higher aspect ratio surfaces, called winglets by Richard Whitcomb.

As for the biplane, the main concepts for winglet theory are summarized below.

\subsubsection{Winglet theory}

P.E. Hemke in his Drag of wings with endplates [11], provided an interesting theory that can explain the advantages and disadvantages of the use of wings with endplates. The contribution of the induced drag for a monoplane can be computed using the following expression from Hemke:

$$
C_{D i}=\frac{C_{L}^{2}}{4} \frac{S}{A_{M}}
$$

Where $A_{M}$ is called area of the equivalent air stream and represents the cross sectional area which includes all air particles that are uniformly deflected downward by the wing and all outside are not deflected. For a monoplane without endplates

$$
A_{M}=\frac{\pi b^{2}}{4}
$$

for a monoplane with endplates

$$
A_{M}=\frac{4 b^{2} K^{2}}{\pi}\left(\frac{2 E}{K}-k^{2}\right)
$$

The ratio of the area of the equivalent air stream of the monoplane to that of the monoplane with endplates is:

$$
R=\frac{\pi^{2}}{4 K^{2}\left(\frac{2 E}{K}-k^{2}\right)}
$$


and it can be calculated as

$$
R=\frac{1}{1+1.66\left(\frac{2 h}{b}\right)}
$$

using $\mathrm{R}$ we can calculate the induced drag as:

$$
C_{D i}=\frac{C_{L}^{2}}{\pi} \frac{R S}{b^{2}}
$$

The use of endplates introduces an additional friction drag given by:

$$
D_{F}=C_{F} q S^{\prime}
$$

where $q$ is the dynamic pressure and $S$ ' the endplates area.

The total drag coefficient is:

$$
C_{D}=C_{D p}+\frac{C_{L}^{2}}{\pi} \frac{R S}{b^{2}}+C_{F} \frac{S^{\prime}}{S}
$$

To obtain the gain with a given set of endplates we subtract from this expression, the expression for drag coefficient for a monoplane without endplates:

$$
\begin{gathered}
\Delta C_{D}=\left(C_{D p}+\frac{C_{L}^{2}}{\pi} \frac{S}{b^{2}}\right)-\left(C_{D p}+\frac{C_{L}^{2}}{\pi} \frac{R S}{b^{2}}+C_{F} \frac{S^{\prime}}{S}\right)=\frac{C_{L}^{2}}{\pi} \frac{S}{b^{2}} \frac{1.66\left(\frac{2 h}{b}\right)}{1+1.66\left(\frac{2 h}{b}\right)}-2 C_{F}\left(\frac{2 h}{b}\right) \\
\Delta C_{D}=\frac{C_{L}^{2}}{\pi} \frac{S}{b^{2}} \frac{1.66\left(\frac{2 h}{b}\right)}{1+1.66\left(\frac{2 h}{b}\right)}-2 C_{F}\left(\frac{2 h}{b}\right)
\end{gathered}
$$

Fig.9 shows for a monoplane with endplates, the reduction of drag against lift coefficient for various values of $2 \mathrm{~h} / \mathrm{b}$, where $2 \mathrm{~h}$ is the height of the end plate. For high values of the lift coefficient, for each value of $2 \mathrm{~h} / \mathrm{b}$, Fig 9 shows a substantial reduction in drag.

It is interesting also to note that in the same fashion, Hemke got the following results, for a biplane with endplates: 


$$
\Delta C_{D}=\frac{C_{L}^{2}}{2} \frac{c}{b}\left(\frac{b^{2}}{A_{M}}-\frac{b^{2}}{A_{M 1}}\right)-C_{F}\left(\frac{2 h}{b}\right)
$$

Where $\mathrm{c}$ is the chord, $A_{M}$ is the area of the equivalent air stream of a biplane without endplates and $A_{M}$ is referred to a biplane with endplates.

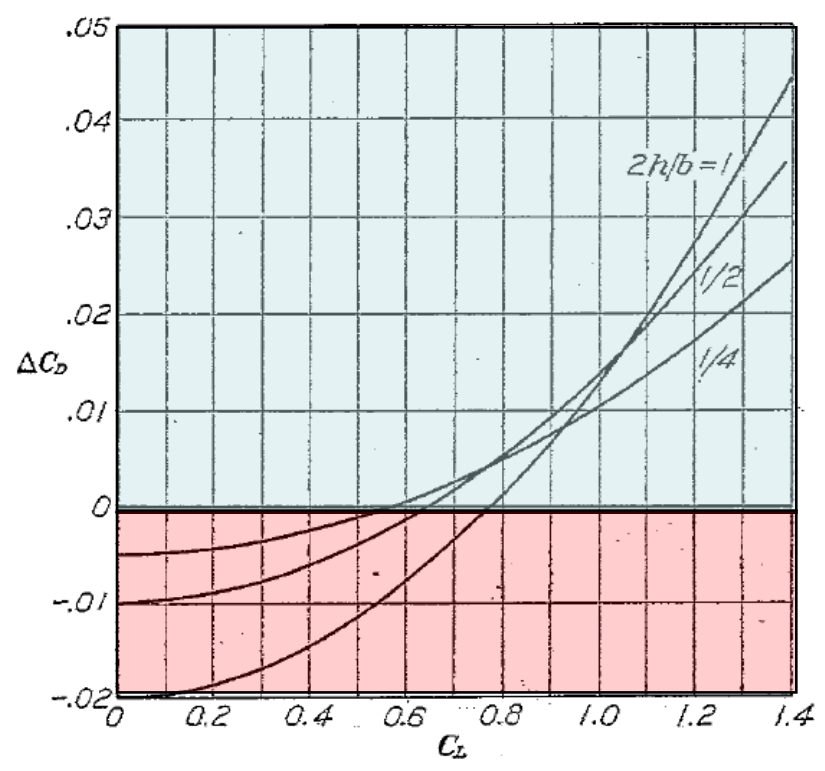

Fig.9 reduction of drag vs $\mathrm{Cl}$ for monoplane with endplates (reproduced from [10]):The graph shows for a monoplane with endplates the reduction of drag vs $\mathrm{Cl}$ for various values of the height of the endplates. For high values of $\mathrm{Cl}$ there is a substantial reduction in $C_{D}$ Monoplane with endplates.

Fig. 10 illustrates the reduction of drag against lift coefficient for various values of $h / G$, where $2 h$ is the height of the end plate, $2 G$ is the gap, Span/chord=6 Gap/chord=1. It shows the interesting result that around a lift coefficient of 0.4 , all the models with various $\mathrm{h} / 2 \mathrm{G}$ start to get a reduction in drag. 


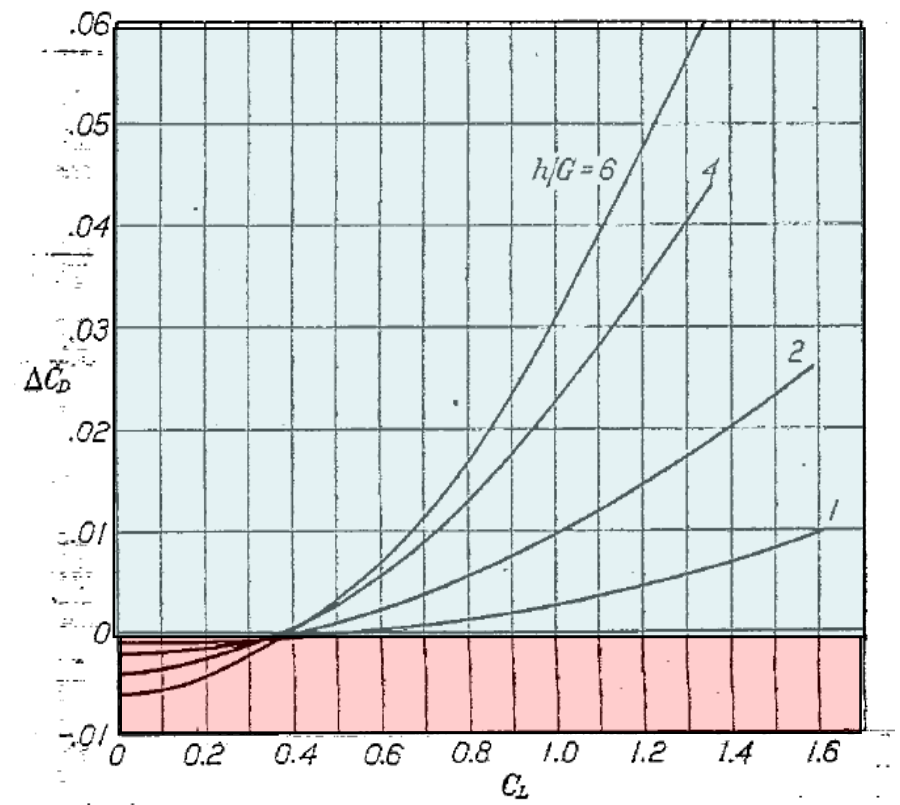

Fig. 10 Reduction of drag vs Cl for biplanes with endplates (reproduced from [10]): The graph shows for a biplane with endplates the reduction of drag vs $\mathrm{Cl}$ for various values of the height 'of the endplates. Around a lift coefficient of 0.4, all the models with various $h / 2 G$ start to get a reduction in drag.

\subsubsection{New winglet concepts}

From the previous theory it is possible to conclude that when the geometric span of the wing is constrained, well-designed winglets do provide significant reduction in airplane drag and have now been incorporated on aircraft ranging from sailplanes to business jets and large commercial transports. The justification for winglets as opposed to span extensions for aircraft that are not explicitly span-limited is less clear. Studies at NASA Langley that compared these two concepts with constrained root bending moment concluded that winglets were to be preferred over span expansion [12]. But studies with constraints on integrated bending moment suggested that the two approaches were almost identical in these respects. 
So the conclusion is that the evaluation of wingtip device advantages must be undertaken for each design again including structural consideration and aeroelastic effects.

Interesting variations on the winglet concept involve staggering the vertical surface longitudinally, called vortex diffuser or multiple high aspect ratio lifting elements attached to the wing tip at several dihedral angles, called wing tip sails. Finally in 1994, Gage [13] produced a complexity evolutionary algorithm, which was allowed to build wings in many individual elements with arbitrary dihedral and optimal twist. Fig.11 shows front views of the population of candidate designs as the system evolves along with the best individual from a given generation. The system discovers winglets and then adds a horizontal extension to the winglet, forming a Clike shape. Further studies showed that the optimal loading on the horizontal extension was downward, reducing root bending moment and providing a positive pitching moment when incorporated on an aft-swept wing. This led to some interesting studies with Boeing on the application of the concept to a blended wing body. 

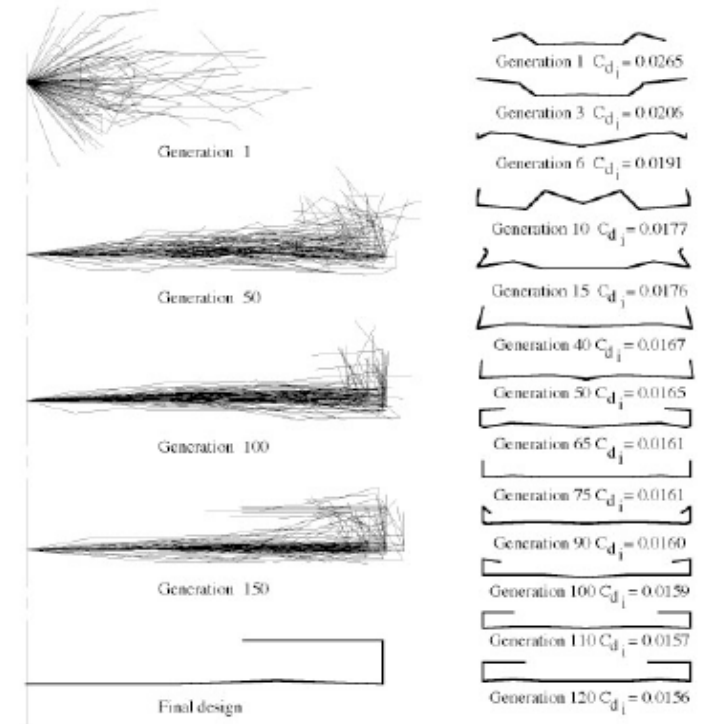

Fig 11 Gage’s complexity evolutionary algorithm: In 1994 Gage [13] produced a complexity evolutionary algorithm, which was allowed to build wings in many individual elements with arbitrary dihedral and optional twist. The system when it evolves discovers winglets and then adds a horizontal extension to the winglets, forming a C like shape as shown in the figure.

\subsubsection{Closed system}

Wings that form closed loops such as the ring wing (Terry 1964, [14]) or boxplane (Miranda 1972, [15]) or joined wing (Wolkovitch 1986, [16]) do not eliminate the tip vortices even though the wing has no tips. Such configurations do claim to possess some interesting properties. The boxplane achieves the minimum induced drag for a given lift, span, and vertical extent as seen before in Fig.5 and a ring wing or joined wing also can achieve span efficiencies greater than 1 due to their nonplanar geometry, but no particular advantage is seen because these configurations are 'closed'. The one feature that does appear in this case, though, is that the optimal load distribution is not unique. One may superimpose a vortex loop with constant circulation on any of these wing geometries. This changes the local loading, but because the circulation is constant, the wake (and hence the lift and drag) is 
unchanged. While this does not reduce the vortex drag for a specified lift, it does provide some design flexibility.

The next section deals with the most famous one, the joined wing configuration and with the Houck Configuration, since the nonplanar configuration under study is very similar to this wing configuration.

\subsubsection{Joined wing}

The “Joined Wing” design was developed principally by Dr. Julian Wolkovitch [16] in the 1980's as an efficient structural arrangement in which the horizontal tail was used as a structural support for the main wing as well as a stabilizing surface. It can be most simply described as two wings joined together at or near the tips; the joined wing has a distinctive diamond shape that is immediately recognized when viewing the wing from either above or in front of the airplane as shown in Fig.12. This shape is obtained by attaching the forward wing low on the fuselage, then sweeping backward and upward while tapering it to the tip. The aft wing is attached high on the vertical fin. It too tapers to the tip but it sweeps forward and downward to join the forward wing.

The diamond shape of the joined wing has the primary structural advantage of strength. This is achieved by each wing bracing the other against the lift loads. In order for this to be beneficial it must be coupled with a significant weight advantage.

According to Dr Wolkovitch [16] a $24 \%$ lighter aircraft may be realized employing the methods and techniques of joined wing design. The truss configuration of the fore wing, aft wing and fin provide a stiffer structure as much as 2.8 times greater than a conventional wing-tail design. Buckling is not a major issue with normal loading applied to the rear wing. Wing torsion is also not a structural problem 
because the rear wing bending stiffness contributes to the front wing torsional stiffness.

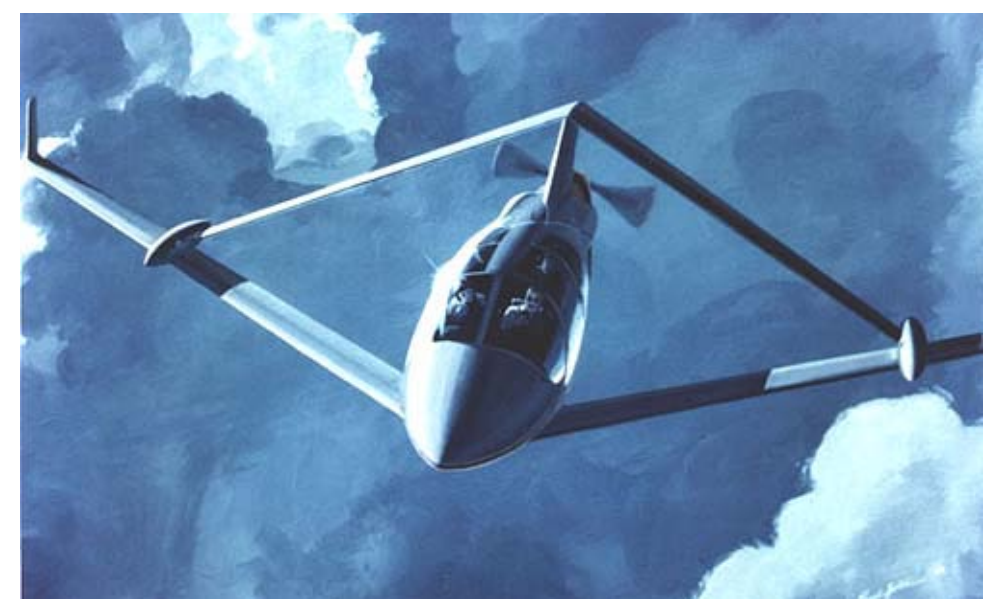

Fig 12 Joined wing configuration: This figure represents a Joined wing configuration. The shape of a joined wing configuration is obtained by attaching the forward wing low on the fuselage, then sweeping backward and upward while tapering it to the tip. The aft wing is attached high on the vertical fin.

The joined wing design also has a potential aerodynamic advantage. Using both wings to produce lift results in less induced drag than conventional wing design for equal lift and span while operating at the same dynamic pressure, according to [16]. However, the joined wing has a disadvantage in terms of parasite drag when compared to a conventional design. This results from a lower wing Reynolds Number and increased ratios of wetted area to lifting area due to a dihedral cosine effect. Other potential disadvantages come from a stability and control characteristics but are not considered here. For this configuration there are, on the other side, potential aeroelastic disadvantages due to the presence of multiple lifting surfaces, aeroelastic characteristics that were recently, and still in the present, under investigation.

This configuration is currently being considered for application to high altitude long endurance UAVs, that usually have high aspect ratio wings. A monoplane can be designed, in fact, with high aspect ratios, $A R$, which allows for more efficient flight. 
But there are limits to how high the aspect ratio can go. Performance-wise, the aircraft loses maneuverability. Structurally, the material is unable to support airframe (i.e. wingspan). The joined-wing aircraft allows for more of an increase in aspect ratio as compared to a monoplane.

\subsubsection{Houck configuration}

The Houck configuration concept was developed by Ronald G. Houck II, owner of Iron Hawk Enterprises LLC. Mr. Houck received a patent and established a sponsor through U.S. Representative David Hobson from the Ohio $7^{\text {th }}$ District. This concept combines an upper and lower wing joined at the tips with flow guides with the intent of significantly reducing vortex losses caused by span wise fluid flow and induced drag. Fig. 13 is a sketch of the Houck Airfoil taken from the United States Patent covering the design. The University of Dayton Research Institute (UDRI) and Iron Hawk Enterprises (IHE) were contracted by the Wright Brothers Institute (WBI) to perform research and development activities on the Houck Configuration for the Air Force Research Laboratory Air Vehicles Directorate (AFRL).

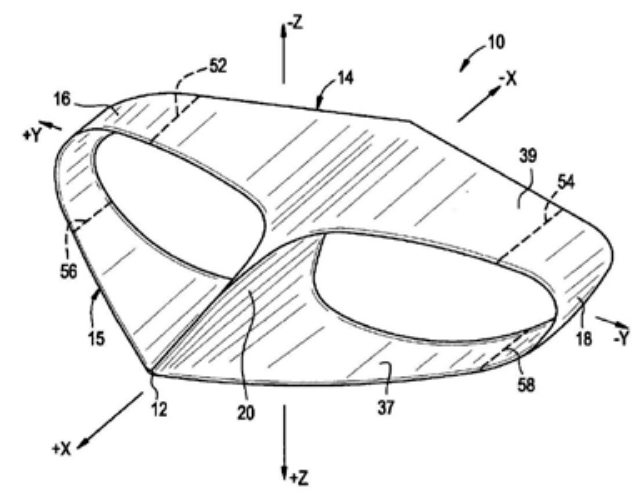

Fig. 13 Houck configuration: This figure represents the Houck Configuration which is the configuration this study inspires to. This concept combines an upper and lower wing joined at the tips with flow guides with the intent of significantly reducing vortex losses caused by span wise fluid flow and induced drag. 
Computer modelling and wind tunnel testing of the concept have been performed to understand the aerodynamic behavior of this configuration. Different configurations in stagger, gap, and curvature of the flow guide that exploit the same concept have been explored.

In an effort to understand the aerodynamic behavior, this configuration has been the subject of some experimental studies. They have been performed by Walker (2007, AFIT (Air Force Institute of Technology)), Oligney and Frash (2007, U.S. Air Force Academy), Killian (2007, AFIT), the University of Maryland (2007, UDM), Landolfo, Genco, Kang, Bichal (2007, UD), [17] and Genco (2008, UD), [18]. Their results will be presented and compared to each other in Chapter VII, with the aim of focusing on the possibility of taking some aerodynamic advantages from the use of a multiple lifting surface configuration similar to the Houck Configuration.

\subsection{Problem Statement}

The aim of this research is to determine if the nonplanar wing under investigation meets the requirements of a Small Reconnaissance Surveillance and Target Acquisition UAV and if so whether there are any structural and/or aerodynamic advantages when compared to a standard monoplane or biplane configuration.

Section 2.2 pointed out the potential advantages and disadvantages of different nonplanar concepts. This research tries to answer if these aerodynamic and structural features can be applied efficiently to the design of a Small UAV.

The importance of an answer to this question lies in the possibility of improving the endurance of this class of aircraft, reducing its weight and therefore its 
stall velocity, or alternatively, increasing the size of the payload which means, in this case, a higher resolution camera. All of these listed potential improvements, as the next chapter will better point out, are main keys in the requirements of a Small Reconnaissance Surveillance and Target Acquisition UAV. 


\section{Chapter III}

\section{Design requirements}

"It's pretty incredible to know what's on the other side of the hill before you go over it ...”[19]

\subsection{Functional requirements}

To design a small, lightweight, affordable man-portable/backpackable and hand launched aircraft capable of providing reconnaissance, surveillance and remote monitoring day and night imagery. Such a small reconnaissance plane can loiter about a ground target over a hill or behind a building, without being detected and without exposing human personnel to harm.

\subsection{Requirements}

1. Weight less than $5 \mathrm{~kg}$

2. Assembled in less than 90 seconds

3. Payload: Dual Forward- and Side-Look EO Camera Nose and Forward- and Side-Look IR Camera Nose interchangeable.

4. Weight of Payload less than $1 \mathrm{~kg}$

5. Span less then 3 meters

6. Hand launched by the operator 
7. Recovered by a belly landing

8. Operating altitude $150 \mathrm{~m}$

9. Climb to the target altitude within approximately one city block (200-500 feet)

10. Rate of climb greater than $150 \mathrm{~m} /$ minute

11. Endurance: more than 1 hour

12. Range: more than $10 \mathrm{Km}$

13. Loiter speed between $12 \mathrm{~m} / \mathrm{s}$ and $20 \mathrm{~m} / \mathrm{s}$

14. Less than 70 decibels acoustic (dBA) at 100 meters distance.

15. Max Gust:6 m/s @ Loiter Speed

\subsection{Specifications}

\section{Portability}

The aircraft and transmission equipment must both be man/backpack portable so that they can be transported and used easily anywhere that surveillance is necessary. In order to reduce the deployment time, the aircraft must be also quickly assembled.

2. Low-profile visibility, low-observability

Due to its low target altitude, to avoid the possibility of being detected by enemies, the feature of small size of the UAV must be pursued throughout the design process. To achieve the same goal, the proposed aircraft must be also able to perform quiet operations.

\section{Weight}

The weight of the UAV must be consistent with the physical resistance and strength of a soldier to transport it during a battle and to throw it for the launch. Therefore the feature of light weight must be pursued during the design process. 


\section{Reliability}

Due to the expensive payload carried by the aircraft, due also to the important data stored during the mission by the aircraft and due to the same high technology product this UAV represents, it is desirable to design a reliable aircraft capable of accomplishing several missions without failures and without being caught by the enemy.

5. Performance: loiter optimization.

The mission goal of the proposed UAV is to provide reconnaissance and surveillance, loitering around the target. An optimization of the loiter time is therefore desirable in order to extend the time this UAV performs its main functional requirement.

\section{Autonomy}

The mission profile requires this UAV to be handled and driven by soldiers, not professional pilots and most of the time during a battle, therefore it must be either easily remotely controlled from the Ground Central Unit or fly completely autonomous missions using GPS waypoint navigation. The UAV also will immediately return to its launch point by selecting the "home" command.

\section{Houck Configuration}

The design of this UAV is part of an effort to explore the possible aerodynamic and structural advantages and disadvantages of a new multiple lifting surface configurations called the Houck Configuration. Therefore this study aims to determine if this particular nonplanar wing concept can meet the requirements of the mission for a small Reconnaissance, Surveillance and Target Acquisition UAV and whether or not there are any advantages to the Houck configuration. 


\section{Chapter IV}

\section{Aircraft Initial Sizing}

\subsection{The State of the Art and Tabulated History}

The age of the small UAV started in 1987, when Dr Paul MacCready's company AeroVironment developed the Pointer, the first hand-launched, backpackcarried UAV. Pointer combined the airframe technology of a high-performance model sailplane with an electric motor and propeller, a consumer video camera and a radio datalink . In 1999, the US Army bought four AeroVironment Pointer [20] small UAVs for testing in the service's "Military Operations in Urban Terrain" and was enthusiastic about the usefulness of the Pointer. The Pointer system was too large to be conveniently carried by soldiers and was normally hauled around in a Hummer vehicle or the like, and so the Army asked AeroVironment if the company could come up with a more portable solution. AeroVironment developed a half-sized control system and a cut-down version of the Pointer called the Raven [21]. The Raven has the same configuration and central pod of the Pointer, but a shorter tail and a wing reduced to $52 \%$ span of $1.34 \mathrm{~m}$. Encouraged by such successes, AeroVironment is also working on a larger version of the Pointer, named the "Puma" [22], with greater endurance and payload.

US forces are also using another mini-UAV in Iraq, the Lockheed Martin Desert Hawk [23]. It weighs 3.2 kilograms (7 pounds), has a wingspan of 1.32 meters 
(52 inches) and a length of 86.4 centimeters (34 inches) but is launched with a bungee cord.

The U.S. Naval Research Laboratory (NRL) has developed a man-portable UAV of roughly the same size as the AeroVironment Raven, named the Dragon Eye [24]. The Dragon Eye is a tailless design with a rectangular wing and twin engines. It is designed to fit into a backpack, with a weight of 2.25 kilograms (5 pounds) and a span of 1.14 meters (3 feet 9 inches). It can be launched by hand or bungee slingshot . An upgrade of the Dragon eye is the AeroVironment Swift.

Another interesting hand launched small UAV taken into consideration in this study is Orbiter [25], an aircraft developed by the Aeronautics Defense Systems in Israel. It has a maximum takeoff weight of $6.5 \mathrm{~kg}$ and can carry a payload of $1.2 \mathrm{~kg}$ weight.

One of the main characteristics of modern small UAVs is loiter time, that's why the main small UAV company AeroVironment are looking at the use of fuel cell batteries, a technology still under development. Last summer, June 29 2007, AeroVironment successfully flew its Puma small-unmanned aircraft for nearly five hours while it was powered by an onboard fuel cell battery hybrid energy storage system.

Under development since 1996 are also a lot of Micro UAVs (less than $30 \mathrm{~cm}$ of span), called MAVs, like the AeroVironment WASP and the Naval Research Laboratory (NRL) MITE. The use of these small airplanes had to wait for the evolution of miniature video cameras and transmitters and other light weight electronic sensors. The Design Requirements listed in Chapter 3 for the proposed mission and in particular the following enhanced requirements: 
- a payload capacity of $1 \mathrm{lb}$ to allow carrying an infrared camera and communications links with a $10 \mathrm{~km}$ range,

- a sufficient power to operate and get stable images from the camera in $6 \mathrm{~m} / \mathrm{s}$ gusts, - an airframe that could stand up to rough treatment in the field, Contributed to the final decision to pursue the idea of designing a Small UAV rather than a MAV since the performance that the requirements desire were beyond the capability of a MAV.

The typical mission profile for this class of aircraft is shown in Fig.14

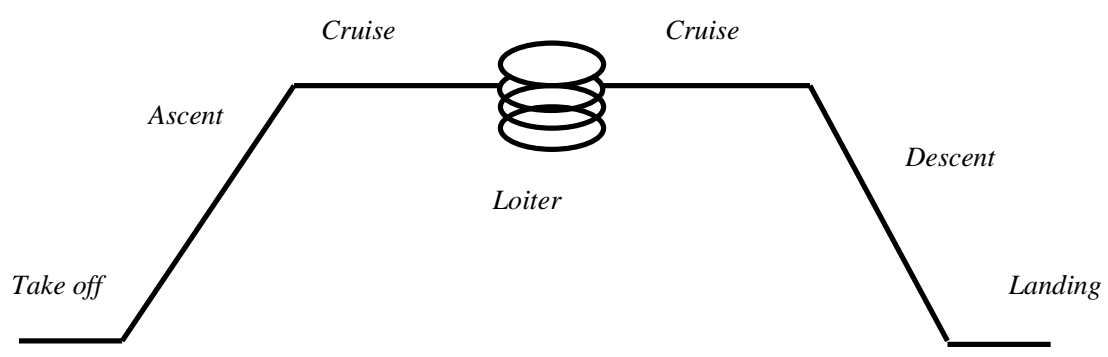

Fig 14 Mission profile of a Small UAVs: Mission profile of a Small UAVs is composed of 6 different steps: After the take off, the airplane climb to the target altitude (ascent); after reaching this altitude there is cruise segment necessary to reach the mission target, then a phase of loitering around the target, finally a cruise to come back to the launching site, a descent and a landing, with or without a parachute.

Using these successful designs of previous Small UAVs and other less famous hand launched UAVs, an historical statistical database for Small UAVs is created here and the primary conceptual design characteristics for this class of aircraft are tabulated and reported in Table 1. Fig. 22 shows some picture of these aircraft designs. 


\begin{tabular}{|c|c|c|c|c|c|c|c|c|}
\hline & & Pointer & Raven & Puma & Dragon eye & Desert Hawk & Orbiter & $\begin{array}{l}\text { Metu } \\
\text { Guventurk }\end{array}$ \\
\hline Span & $m$ & 2.7 & 1.4 & 2.6 & 1.1 & 1.32 & 2.2 & 2.2 \\
\hline Length & $m$ & 1.8 & 1.1 & 1.8 & 0.9 & 0.86 & 1 & 1.35 \\
\hline Weight TO & $\overline{K g}$ & 4.095 & 1.9 & 5.5 & 2.3 & 3.2 & 5.5 & 4.5 \\
\hline Weight PL & $\mathrm{kg}$ & 1 & 0.5 & 0.9 & 0.5 & 0.5 & 1.2 & 0.5 \\
\hline Surface & $m^{2}$ & 0.75 & 0.3 & 0.832 & 0.363 & 0.28 & 0.611 & 0.7 \\
\hline Power & Watt & 300 & 250 & 600 & 400 & 300 & 400 & 900 \\
\hline Velocity_Stall & $\mathrm{m} / \mathrm{s}$ & 8.4 & 8.68 & 9.22 & 8.5 & 11.43 & 12.75 & 9 \\
\hline A.R & & 10 & 6.7 & 9 & 3.33 & 6.28 & 7.6 & 7.5 \\
\hline Endurance & $\min$ & 90 & 80 & 120 & 45 & 60 & 2.5 & 90 \\
\hline Wp/Wto & & 0.24 & 0.26 & 0.16 & 0.217 & 0.15 & 0.27 & 0.11 \\
\hline hp/W & $h p / N$ & 0.01 & 0.0134 & 0.014 & 0.0237 & 0.0128 & 0.0099 & 0.027 \\
\hline WIS & $\mathrm{N} / \mathrm{m}^{2}$ & 53.56 & 62.13 & 64.84 & 62.16 & 112 & 104.25 & 63.06 \\
\hline
\end{tabular}

Table 1 UAV previous successful designs: This table reports per each row the primary conceptual design characteristics for 7 different successful UAV designs. This table is very important to start to have a feeling for the order of magnitude of these characteristics for this class of aircraft.

\subsection{Constraint equations}

To better define a feasible range of values and to provide some initial numbers to be used in the sizing of the proposed UAV, a plot, called a constraint diagram, showing the relationship between thrust-to-weight ratio (T/W) and wing loading (W/S) for the required mission was developed using the equations outlined below. In particular the term thrust-to-weight is associated with jet-engined aircraft. For propeller-powered aircraft the equivalent term is expressed in terms of horsepower, and is the horsepower-to-weight ratio. 
For a Propeller Driven Aircraft the main constraint equations are:

Maximum Load/Turn

$$
\frac{H P}{W}=\frac{1}{550 \eta_{p}}\left[\frac{1}{2} \rho V^{3} C_{D_{o}}\left(\frac{S}{W}\right)+2 K \frac{n^{2}}{\rho V}\left(\frac{W}{S}\right)\right]
$$

Endurance

$$
\frac{H P}{W}=\frac{4}{550 \eta_{p}} C_{D_{o}}^{1 / 4}\left(\frac{K}{3}\right)^{3 / 4}\left(\frac{2}{\rho} \frac{W}{S}\right)^{1 / 2}
$$

Cruise

$$
\frac{H P}{W}=\frac{2}{550 \eta_{p}} C_{D_{o}}^{1 / 4} K^{3 / 4}\left(\frac{2}{\rho} \frac{W}{S}\right)^{1 / 2}
$$

(3)

\section{Takeoff Distance}

$$
\frac{H P}{W}=\frac{2.44}{550 \eta_{p}} \frac{1}{g d_{t o}}\left(\frac{1}{\rho_{S L} C_{L_{\max }}} \frac{W}{S}\right)^{3 / 2}
$$

Stall Condition

$\frac{W}{S}=\frac{\rho}{2} C_{L_{\max }} V_{S o}^{2}$

Using the greater knowledge about this class of aircraft derived from analyzing comparative aircraft and the historical assessment, it is possible to make some first crude assumptions about the parameters the constraint equations require: 


$$
\begin{aligned}
& \rho_{S . L}=1.225 \frac{\mathrm{Kg}}{\mathrm{m}^{3}} \\
& \rho @ 150 \mathrm{~m}=1.20 \frac{\mathrm{Kg}}{\mathrm{m}^{3}} \\
& \eta_{p}=0.7 \\
& V=15 \frac{\mathrm{m}}{\mathrm{s}} \\
& n=2.5 \\
& C_{D 0}=0.035 \\
& A . R .=7.8 \\
& e=0.8 \\
& K=\frac{1}{\pi A . R . e}=0.051 \\
& C_{L M A X}=1.3 \\
& d_{\text {TO }}=14 \mathrm{~m} \\
& V_{\text {STALL }}=9 \frac{\mathrm{m}}{\mathrm{s}}
\end{aligned}
$$

The values of density at sea level and at the target altitude are tabulated in the standard atmosphere tables [7].

As it will be explained later in this chapter, the decision of using an electric motor was made; the efficiency of a combined motor and propeller can be first estimated [26] equal to 0.7 .

The loiter velocity for Small reconnaissance and surveillance UAV is usually constrained by the camera's parameters and, at a target altitude of $150-200 \mathrm{~m}$ the maximum speed to avoid blurry images is around $15 \mathrm{~m} / \mathrm{s}$.

The mission profile for the Small UAV considered usually requires a maximum load factor $n$ related to the maximum level turn bank angle (65 deg) used during the loiter. As it will be demonstrated in subsequent chapters, during this kind of manoeuvre the load factor is around 2.5. 
The parasite drag that consists mostly of a skin friction component and a component due to pressure depends on the particular layout of the aircraft and its protuberances but at this early point of the design it is possible to assume that it should range between 0.025 and 0.04 . A value of 0.035 was considered.

From the tabulated history, excluding the Dragon Eye, which is a twin prop, launched by a bungee cord, an average value of the A.R. for this class of aircraft is 7.8 .

For the lack of a more accurate Oswald efficiency factor for this class of aircraft, a typical value for standard aircraft was chosen, equal to 0.8 .

The maximum lift coefficients for a wing configuration without the use of high-lift devices like flaps and slats at a Reynolds number around 400,000 is fixed by the current technology and a typical value is 1.3 . The stall velocity historically can be estimated around $9 \mathrm{~m} / \mathrm{s}$.

Finally, since the proposed UAV is hand launched, an equivalent takeoff distance was considered: it represents, in this case, the equivalent distance that an aircraft should cover in order to reach the required velocity to takeoff if the required velocity to takeoff is the stall velocity and the acceleration is the acceleration that a soldier can give to the aircraft running with a weight of about $4 \mathrm{~kg}$ in his hand. This distance was estimated to be approximately 14 meters. A sensitivity analysis is later performed in order to take into consideration the impact of any errors due to the assumptions made.

Fig. 15 shows the constraint diagram obtained using the previous assumptions. 


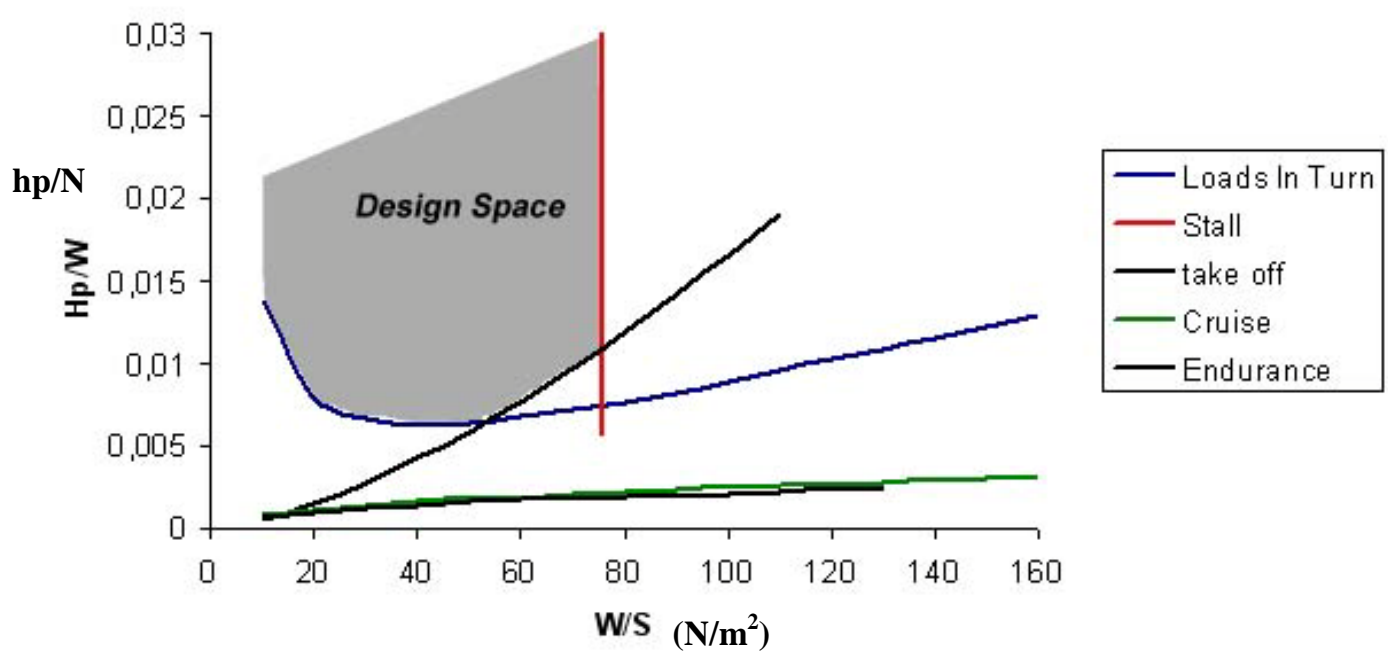

Fig. 15 Constraint diagram: This figure shows the constraint diagram obtained plotting the constraint equations. This graph is very useful because each point of the design space in grey, represents a possible aircraft design for the class of aircraft and for the profile mission in consideration, based on physics alone

It is noted that the cruise and endurance constraints for this kind of mission are less important than the others. This diagram is very important because it shows that any point in the design space represents a possible aircraft design for the required mission based on physics alone.

If this is true then it becomes interesting to plot the points representing each Small UAV whose data were tabulated in the previous section on this diagram, as shown Fig. 16. 


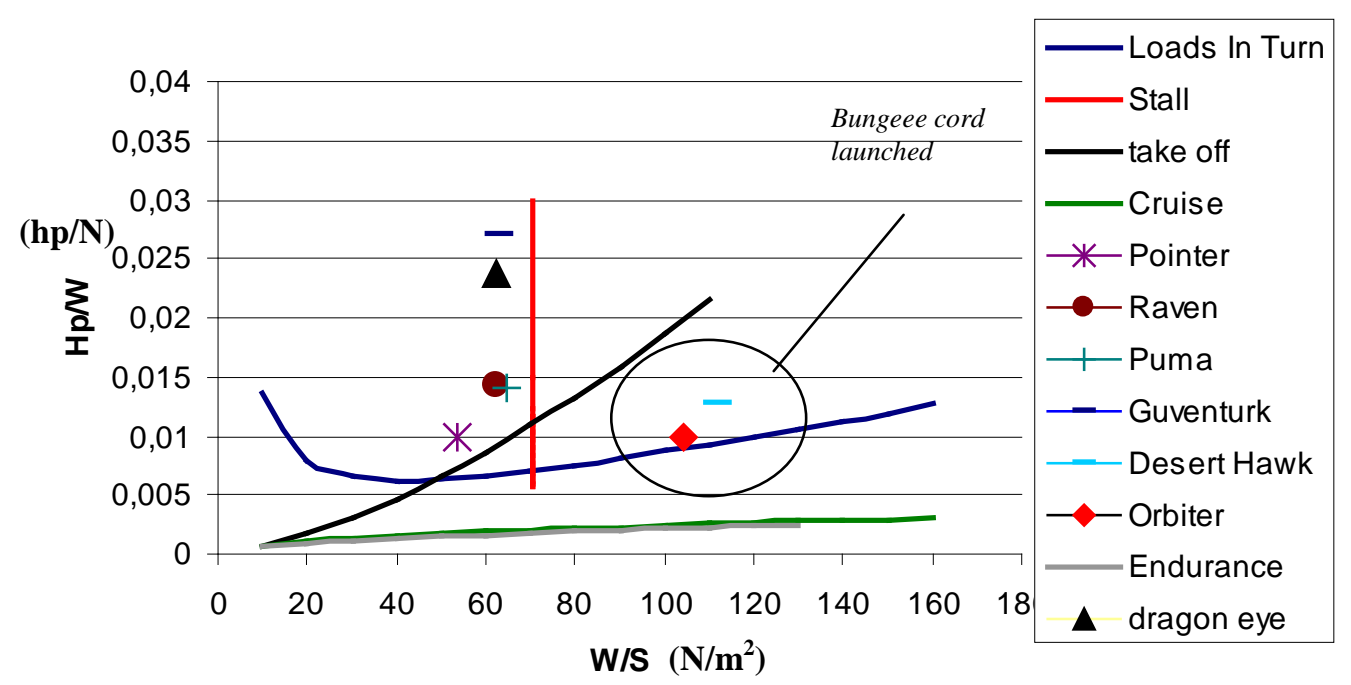

Fig. 16 Constraint diagram and previous designs: This figure shows the constraint diagram for the class of aircraft under consideration obtained plotting the constraint equations and the design points of previous small UAV. It can be noticed that bungee cord launched UAVs have different assumptions, like different stall velocity and therefore the points which represent these configuration in this graph fall outside the design space.

Fig.16 confirms the assumptions made were sufficiently accurate, since all the UAV designs considered are located in the design space, with the exception of the 2 bungee cord launched UAVs. Obviously the assumptions made for the hand launched aircraft don't remain valid for a bungee cord launched UAV. For this other class of aircraft the stall velocity and the equivalent takeoff distance will be greater.

\subsection{Sensitivity analysis.}

A sensitivity analysis is performed to study how the variation in the design space can be apportioned to the variation of different parameters. In other words, to acquire a better knowledge in order to make an informed decision and select a realistic design point in the constraint diagram, it is necessary to identify the critical 
assumptions made and therefore the factors that contribute most to the output variability.

The biggest sources of uncertainty come from the assumptions made for the values for $C_{D 0}, d_{T O}, C_{L M A X}$ for this class of aircraft as well as the stall speed. Considering a variation of $\pm 15 \%$ for these parameters it is possible to obtain the results seen in Fig.17 that show the variation of the size and shape of the design space with variation of $C_{D 0}$.
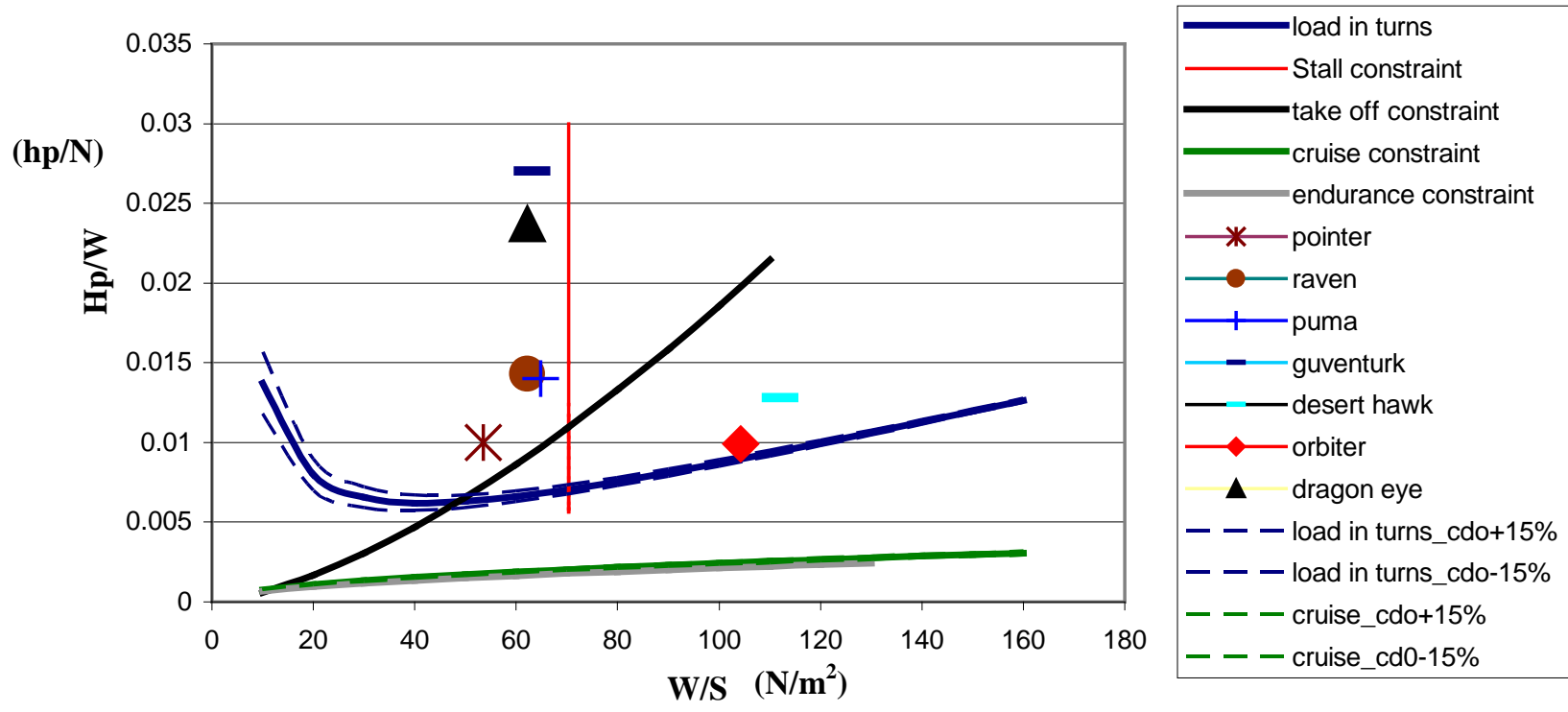

Fig.17 Sensitivity analysis $C_{D 0}$ variation: This figure shows the constraint diagram for the class of aircraft under consideration, obtained plotting the constraint equations and it also shows how the variation of $C_{D 0}$ effects the variation of the design space. It is noticed that this variation has a small effect on the design space

Fig. 17 shows a variation of the load in turns constraint and of the range and endurance constraints with variation in $C_{D 0}$. It is noted that the variation of $C_{D 0}$ has a small effect on the design space. Therefore we can conclude that at this early point in the conceptual design, the choice of a $C_{D 0}=0.03$ can be used, to be refined later in the design process. 
Fig. 18 shows the variation in the design space with the variation in $d_{T O}$.

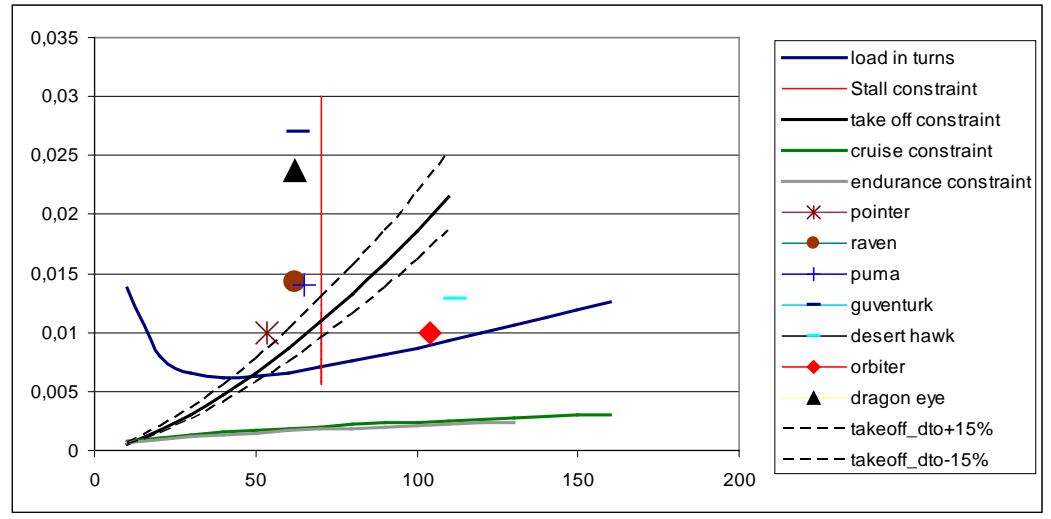

Fig.18: Sensitivity analysis $d_{\text {TO }}$ variation: This figure shows the constraint diagram for the class of aircraft under consideration, obtained plotting the constraint equations and it also show the variation of the design space with the variation of $d_{T O}$. It is noticed that this variation has an important effect on the design space.

Fig. 18 shows $d_{T O}$ is an important factor that affects the size of the design space of a hand-launched aircraft. The most constraining curve, as would be easily predicted, is the takeoff constraint. It shows also that an estimate of $d_{T O}$ less than the $15 \%$ of the initial estimated $d_{T O}$ represents a signal of a potential wrong assumption, since historically some of the UAVs could physically not fly under those conditions.

Fig. 19 shows the variation in the design space due to a variation in the $C_{L \text { MAX }}$. It is noted that the most constraining curves are the stall constraint and the takeoff constraint. The effects of a variation of $C_{L M A X}$ are significant since this entire class of UAVs cannot fly with a $C_{L M A X}$ equal and less than:

$C_{L M A X \text { modified }}=C_{L_{M A X}}-0.15 C_{L M A X}=1.1$.

This is due to the hand launch feature, which requires a high $C_{L M A X}$ to liftoff without flaps. This observation will drive the choice of an airfoil that satisfies this constraint 
in a more advanced phase of the process, On the other side, Fig.19 shows also that for this range of Reynolds number and for small UAV without flaps, a $C_{L M A X}$ greater than 1.4 is quite rare. This means that it represents a fixed real technological constraint to keep in mind in the design of the aircraft.

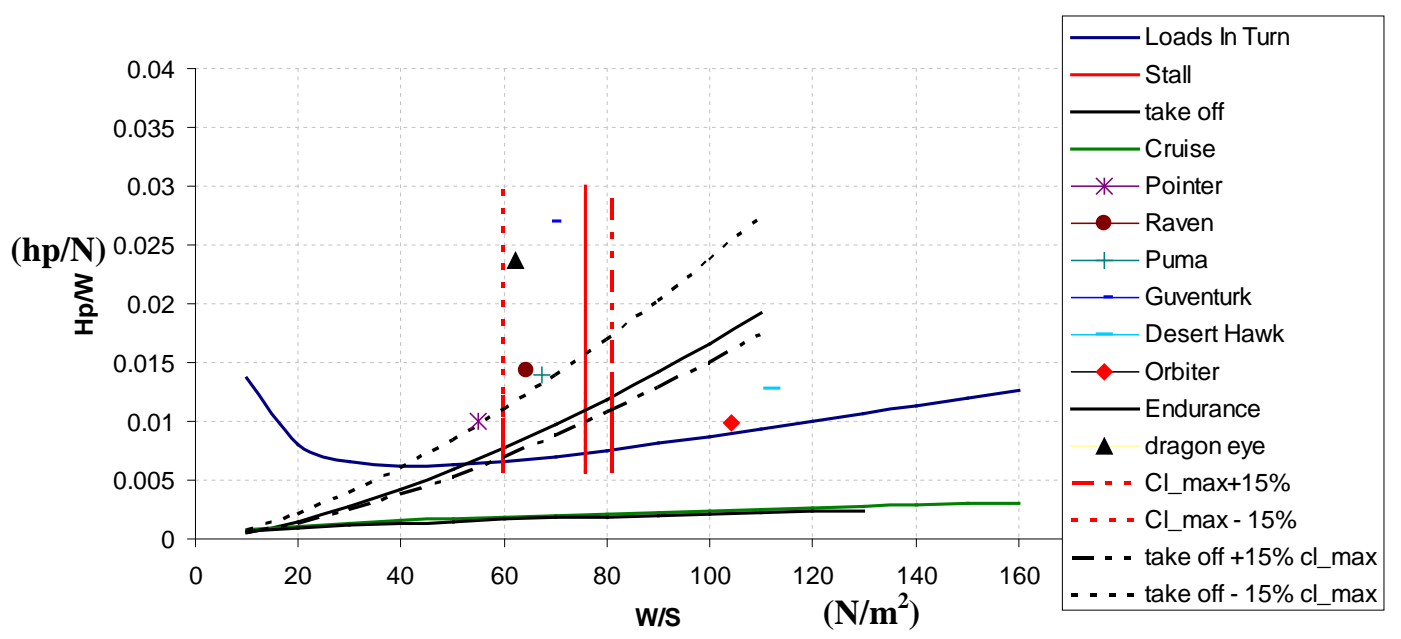

Fig.19: Sensitivity analysis: $C_{L M A X}$ variation: This figure shows the constraint diagram for the class of aircraft under consideration, obtained plotting the constraint equations and it also shows the variation of the design space with the variation of the $C_{\text {LMAX }}$. This variation has a huge impact on the design space and in particular on the stall and take off equations.

The last factor take into account is the stall velocity. (Fig.20)

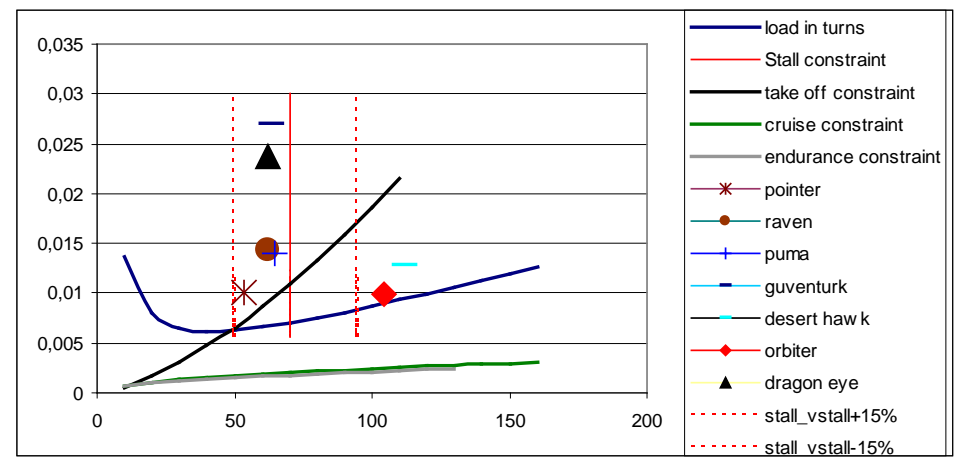

Fig.20 Sensitivity analysis: stall speed variation: This figure shows the constraint diagram for the class of aircraft under consideration, obtained plotting the constraint equations and it also shows the variation of the design space with the variation of the stall speed. In the same way of the max lift coefficient, the stall velocity has a huge impact on the design space too. 
Clearly the stall velocity has a huge impact on the design space, and therefore it represents a critical assumption. This was also widely predictable since for this class of aircraft the stall speed represents a strong constraint due to potential hand launched criterion. Even a slight increase in stall speed for this class of aircraft represents the possibility of carrying a bigger payload. This also explains why often the hand launched criterion is rejected, and another method of launch preferred, for example the bungee cord method. Refining the assumptions made at the beginning on the basis of bungee cord launched UAVs:

$\rho_{S . L}=1.225 \frac{\mathrm{Kg}}{\mathrm{m}^{3}}$

$\rho @ 150 m=1.20 \frac{\mathrm{Kg}}{\mathrm{m}^{3}}$

$\eta_{p}=0.7$

$V=20 \frac{m}{\mathrm{~s}}$

$n=2.5$

$C_{D 0}=0.035$

A.R. $=7.8$

$e=0.8$

$K=\frac{1}{\pi A \cdot R \cdot e}=0.051$

$C_{L M A X}=1.3$

$d_{\text {TO }}=30 \mathrm{~m}$

$V_{\text {STALL }}=12.5 \frac{\mathrm{m}}{\mathrm{s}}$

it is interesting to note the change in the design space (Fig.21). 


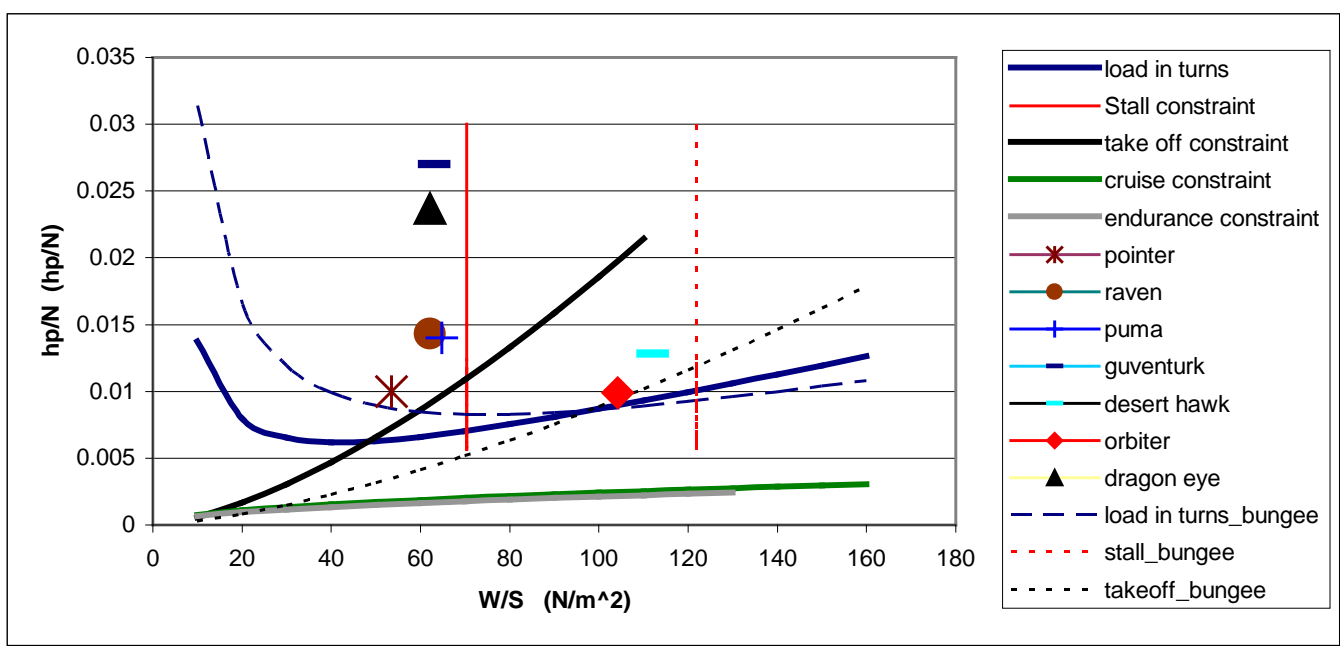

Fig.21 Sensitivity analysis: bungee cord launched aircraft: This figure shows the constraint diagram for a bungee cord launched aircraft obtained plotting the constraint equations modified for a bungee cord launched aircraft. It is noticed that now the new design space contains also the 2 bungee cord aircrafts.

Even though a bungee cord launched UAV provides many advantages, like a bigger design space and the possibility to carry more payload, the disadvantages due to the presence of additional equipment to transport and deploy and due to the additional weight of the aircraft are still greater than the advantages.

Once the quality of the assumptions has been verified and a better understanding has been acquired, it is possible to proceed towards a new step of the design process, the initial sizing process. In the next page, reports some pictures of the comparative Small UAVs taken into consideration in this chapter. 

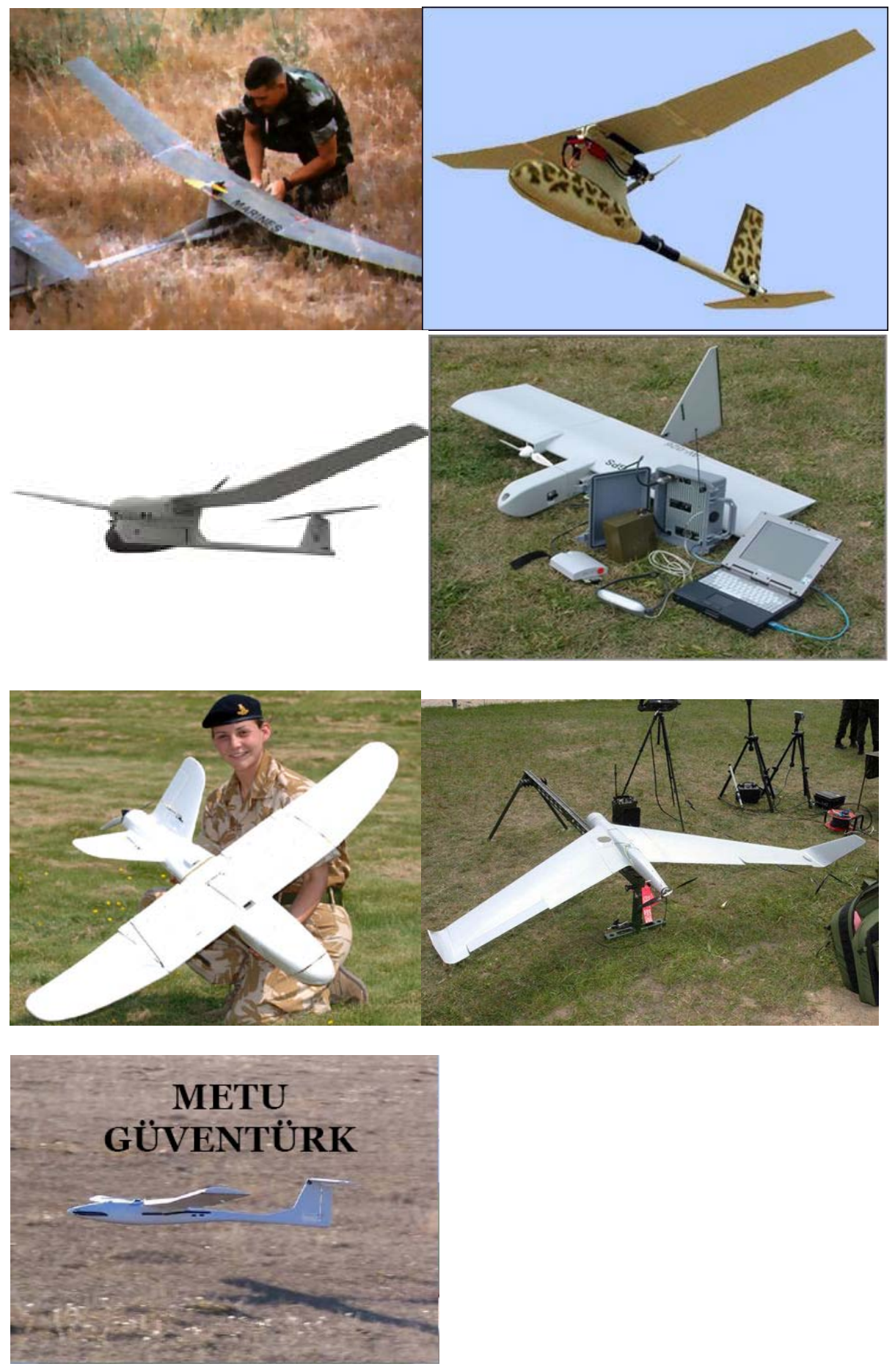

Fig. 22 Comparative UAVs: Photos of comparative small UAVs: these photos can help to have a visual understanding of the class of aircraft under consideration. From the top left to the bottom the pictures represent: Pointer, Raven, Puma, Dragon Eye, Desert Hawk, Orbiter and Metu Guventurk. From this pictures it can be also noticed the different layouts used for the different small UAV. 


\subsection{Initial sizing}

\subsubsection{Payload Weight Fraction}

In order to have a first estimate of the Weight at Takeoff of the airplane it is useful to consider the historical values of the Payload Weight Fraction reported in Table 1 and highlighted in Table 2:

\begin{tabular}{|l|l|r|r|r|r|r|r|r|}
\hline & & Pointer & Raven & Puma & Dragon eye & Desert Hawk & Orbiter & Guventurk \\
& & & & & & & & \\
\hline Weight TO & $\mathrm{Kg}$ & 4.095 & 1.9 & 5.5 & 2.3 & 3.2 & 5.5 & 4.5 \\
\hline Weight PL & $\mathrm{kg}$ & 1 & 0.5 & 0.9 & 0.5 & 0.5 & 1.2 & 0.5 \\
\hline Wp/Wto & & 0.24 & 0.26 & 0.16 & 0.217 & 0.15 & 0.27 & 0.11 \\
\hline
\end{tabular}

Table 2 Historical value: Historical values of Payload Weight Fractions. From this Table, Orbiter and

Puma have the highest Payload fraction which is a predictable result: Pointer is an old UAV and at that time the payload was heavier than the modern miniaturized cameras, Orbiter is a bungee launched aircraft and therefore has a higher stall speed and therefore can carry a higher payload keeping constant all the other parameters.

The average value of the Payload Weight Fraction results:

$$
\frac{W_{P}}{W_{\text {TO }}}=0.201
$$

Considerable thought and research goes into the overall selection of the payload since it is imperative that the payload selected is efficient and will satisfy the mission requirements in order to insure a successful product. Besides, as it is shown in this section, the payload is also the driving factor behind the design and sizing of the aircraft. 
After a first research, in order to deliver colour or infrared imagery to the ground control and remote viewing stations in real time a dual forward and side look EO/IR camera was chosen. The initial Payload Weight was estimated to be, for a medium-high price camera including cables:

$$
W_{P L}=0.6 \mathrm{Kg} .
$$

Therefore, using the Historical Values, it is possible to estimate an initial value of the Weight at Takeoff of the proposed UAV:

$$
W_{T O}=\frac{W_{P L}}{0.201}=\frac{0.5}{0.201} \approx 3 \mathrm{~kg}
$$

\subsubsection{Design Point}

Once the Takeoff Weight is known, using the Horsepower to Weight Ratio vs. Wing Loading diagram it is possible to determine the Wing Surface Area and the Thrust of the airplane.

In order to pick one point in the design space of Fig. 15 that represents the proposed aircraft for the required mission it is important to have a clear understanding of how these 2 parameters affect the performance of the aircraft.

An aircraft with a higher hp/W will accelerate more quickly, climb more rapidly, reach a higher maximum speed and sustain higher turn rates. On the other side, a bigger engine will consume more fuel or battery, which will drive up the aircraft's takeoff weight to perform the required mission.

The Wing Loading, the weight of the aircraft divided by the area of the reference wing, affects stall speed, climb rate, takeoff and landing distances and turn performances. The wing loading also determines the design lift coefficient and affects the drag because of the wetted area and wing span. Finally, wing loading affects the 
takeoff weight of the airplane. A low Wing Loading generally means a larger wing; this may improve the performance, but the additional drag due to the larger wetted area and the additional empty weight due to the larger structure of the wing will increase takeoff weight to perform the required mission. A compromise between $\mathrm{hp} / \mathrm{W}$ and W/S must be found that better matches and optimizes the required mission of the aircraft.

An important aid that can drive the choice of the design point can arrive from the study of the design points of comparative aircraft able to perform the same mission. Any trend or tendency must be taken into consideration.

Considering the requirements of the proposed mission, a lightweight, long endurance and short takeoff distance airplane is highly desirable. Looking at the design space in Fig.16 as an initial estimate, the following combination of values seemed to be a good compromise that respects the constraints:

$$
\begin{gathered}
\frac{W}{S}=61 \mathrm{~N} / \mathrm{m}^{2} \\
\frac{h p}{W}=0.01 \mathrm{hp} / \mathrm{N}
\end{gathered}
$$

In making this decision the potential development of the future technology state of the art was also taken into consideration; in other words it was estimated that in the near future the continuous research in the battery field will bring some small new improvements that will be ready for use at the time the aircraft is built. This allows the selection of a point with the same W/S that the comparative SUAVs but with a little bit smaller hp-to-weight ratio. The design point chosen is reported in Fig.23. 


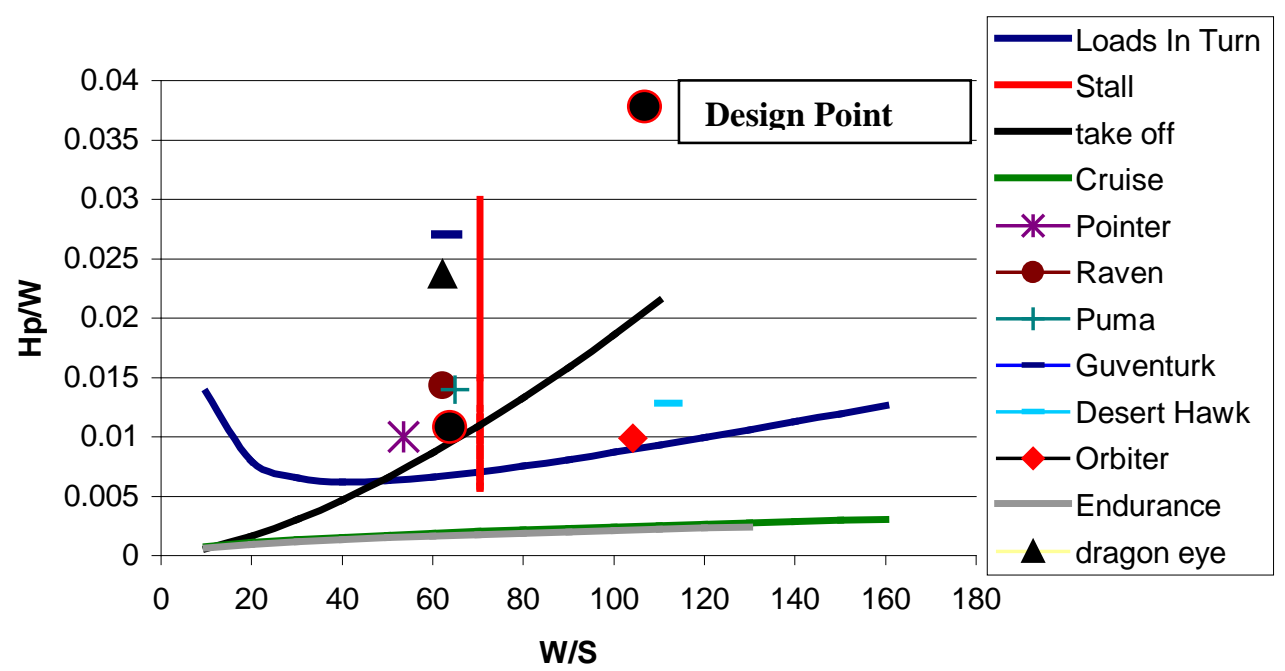

Fig.23 design point: This figure shows the constraint diagram obtained plotting the constraint equations and the design point of previous small UAV and it shows the chosen design point. This design point should meet the requirements of the proposed mission: a lightweight, long endurance and short takeoff distance airplane.

\subsubsection{Wing geometry sizing}

Once the Wing Loading and the Takeoff Weight have been calculated, it is possible to determine the Wing Surface Reference Area:

$$
S=\frac{W}{W / S}=\frac{3 K g}{61 N / m^{2}}=0.48 \mathrm{~m}^{2}
$$

Assuming an initially rectangular wing, the aspect ratio can be defined as simply the span divided by the chord. A wing with a high aspect ratio has tips farther apart than an equal area wing with a low aspect ratio. Therefore, the amount of the wing affected by the tip vortex, due to the 3D effects that tend to lower the pressure difference between the upper and lower surface, is less for a high-aspect-ratio wing than for a low-aspect-ratio wing. In other words a high aspect ratio wing does not experience as much of a loss of lift and increased drag due to tip effects as a low- 
aspect-ratio wing of equal area. On the other hand a higher aspect ratio will generally increase the structural weight of the wing and therefore of the airplane due to the increased stresses the wing undergoes to. Again a compromise must be reached. Historical values and statistical trends can help in this decision. Fig.24 reports the historical trend of the Weight vs. the A.R.

\section{A.R. vs. Weight}

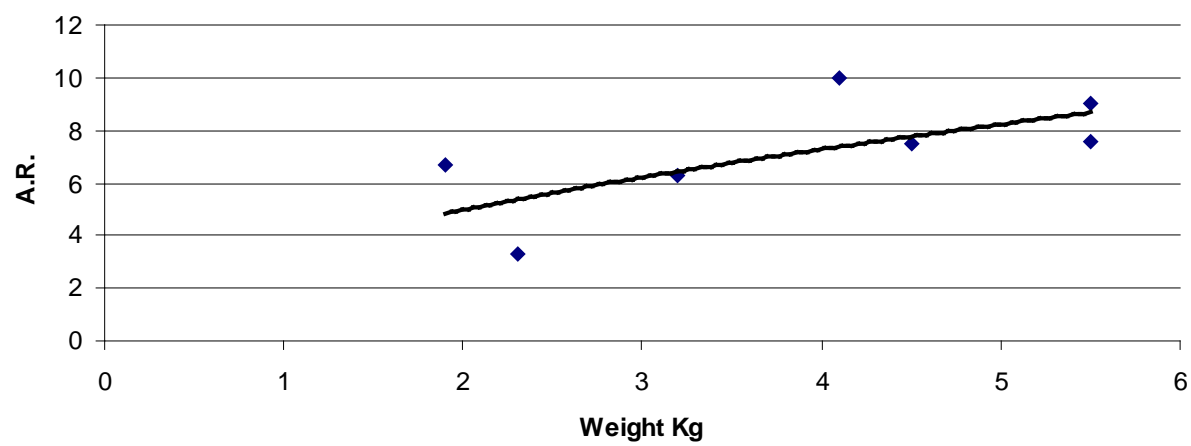

Fig.24 AR vs weight for historical airplanes: this graph shows the A.R. vs. weight of historical airplanes of the same class of the proposed one. It confirms an increase in A.R. generally increases the weight of the SUAV.

The trend line of Fig. 24 confirms that an increase in A.R. generally produces an increase in Takeoff weight. Considering the graph in Fig.24 but assuming also that the use of composites and fibres, and the potential use of the Houck Configuration, will probably decrease the overall structural weight of the wing, an A.R. of 8 was chosen.

The resulting span and chords can be easily calculated:

$$
\begin{gathered}
S=b \cdot c=0.48 \mathrm{~m}^{2} \\
\text { A.R. }=\frac{b}{c}=8
\end{gathered}
$$

Therefore 


$$
\begin{aligned}
& \mathrm{c}=0.24 \mathrm{~m} \\
& \mathrm{~b}=1.96 \mathrm{~m} .
\end{aligned}
$$

\subsubsection{Operating lift coefficient}

It is possible at this point to calculate an operating lift coefficient. The aircraft should be designed so that it flies the design mission at or near the operating lift coefficient. In level flight the lift must equal the weight, so the required lift coefficient can be found as follows:

$$
\frac{1}{2} \rho V^{2} S C_{L}=W \rightarrow C_{L}=\frac{W}{\frac{1}{2} \rho V^{2} S}=0.25
$$

Where the following values, estimated in the previous sections, were used:

$$
\begin{gathered}
\frac{W}{S}=61 \mathrm{~N} / \mathrm{m}^{2} \\
\rho=1.2 \mathrm{Kg} / \mathrm{m}^{3} \\
\mathrm{~V}=20 \mathrm{~m} / \mathrm{s}
\end{gathered}
$$

\subsubsection{Lift to Drag Ratio}

It is useful at this point to have a first estimate of the Lift to Drag Ratio, L/D, and curve. Under the assumption of flat plate airfoil,

$$
C_{L}=2 \pi \alpha
$$

The drag coefficient of an aircraft, as anticipated in the Literature Review, can be broken into 2 main parts: Parasite Drag Coefficient, $\mathrm{C}_{\mathrm{DO}}$, and the Induced Drag Coefficient, $\mathrm{C}_{\mathrm{Di}}$. Besides 


$$
C_{D i}=\frac{C_{L}^{2}}{\pi e A R}
$$

Under the assumptions made in section 4.2 and 4.4 .3 that

$$
\begin{gathered}
\mathrm{C}_{\mathrm{DO}}=0.03 \\
\mathrm{e}=0.8 \\
\mathrm{AR}=8
\end{gathered}
$$

and under the assumption of considering angles of attack lower than the stall angle, it is possible to draw an initial draft of the Lift to Drag versus the angle of attack curve (see Fig. 25).

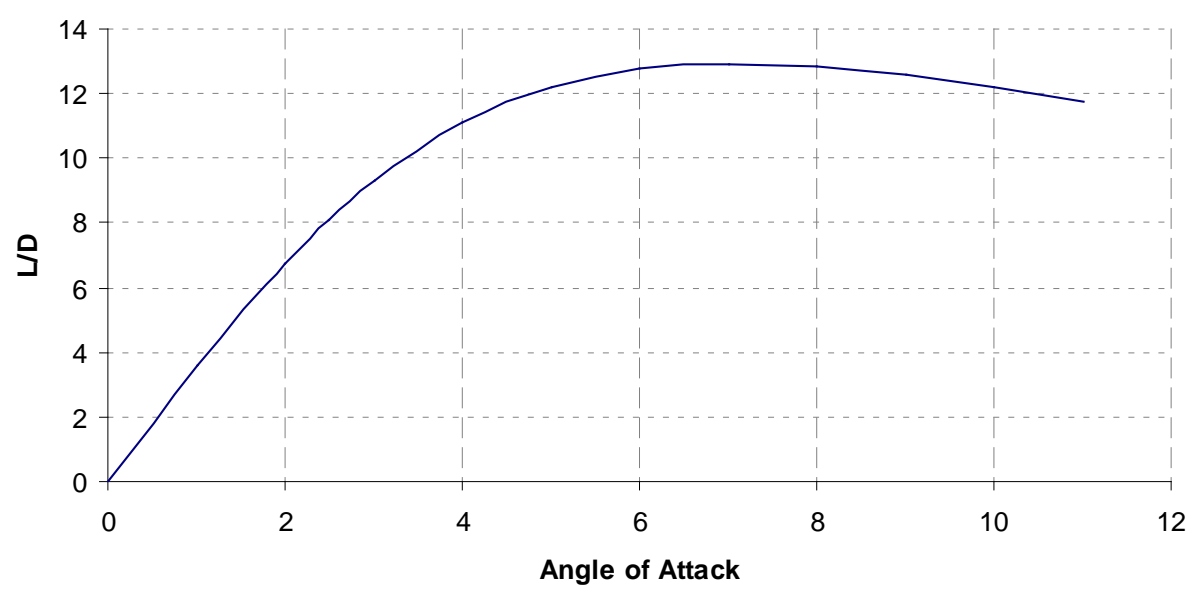

Fig.. 25 L/D vs Angle of attack: This graph shows the L/D versus angle of attack for the proposed aircraft. This graph is only an initial draft because it is based on the assumptions made in section 4.2 and 4.4.3 and the assumption of low angles of attack.

For an operating lift coefficient, $\mathrm{C}_{\mathrm{L}}$, equal to 0.25 , that corresponds to an angle of attack of 2.3, the $\mathrm{L} / \mathrm{D}$ is

$$
\mathrm{L} / \mathrm{D}=7.6
$$




\subsubsection{Propulsion}

Small air vehicle propulsion must be as lightweight and efficient as possible. As long as there is no hover requirement, fixed-wing propeller-driven SUAVs have been found to be the most energy efficient. High energy density and high power density are the most important characteristics. The energy densities of available storage systems are [26] 47,000 J/g for gasoline; 23,000 J/g for methanol, $360 \mathrm{~J} / \mathrm{g}$ for lithium batteries, and $100 \mathrm{~J} / \mathrm{g}$ for NiCad batteries. Although gasoline has an energy density more than 100 times that of lithium batteries, the decision of an electric powered aircraft was taken because in addition to more rapid and continuing technological improvement, electric power provides:

1) High reliability. Internal combustion engines are more difficult to start and cannot be restarted in flight. This feature meets the reliability specification listed in Chapter 3;

2) Reduced noise and vibration. The vibration issues of the internal combustion engines can reduce the quality of the images that is the final payload objective. Besides for Small UAVs that are intended for unobtrusive or low-observability operations, such as close-in military reconnaissance, the advantages of quiet operation and low infrared signature compared with internal combustion engines are particularly important. These features meet the Low-profile visibility specification, listed in Chapter 3.

3) Precise power management and control

4) The choice of an electric motor avoids soldiers having to carry volatile and explosive fuel on their back and eliminates lubricating oil spray. 
However internal combustion engines have been used by early designers especially by those from the model airplane community.

\subsubsection{Batteries}

The primary disadvantage and limitation of battery-powered flight, as mentioned before, is the relatively low specific energy (stored energy per unit weight, normally stated in watt hours per kilogram) of batteries. This translates to a much higher "fuel" weight requirement for electric-powered vehicles, which made electric aircraft flight impractical prior to 1970s. Battery technology, however, has improved significantly since that time and continues to improve at an accelerating pace.

Recent flight experiments with fuel cells suggest a possible alternative to battery power. Fuel cells combine a fuel catalytically with oxygen to produce electric power directly, without combustion. Thus the energy store for a fuel-cell system can be extremely high specific-energy fuel, generally hydrogen. Fuel cells might be generally available in the future but they were not taken into consideration in this research.

For the proposed electrical aircraft the decision was taken to use lithium-ion-polymer (LiPo) cells, which offer both high specific energy and power. They are rechargeable and offer 5 to 10 times the specific energy of the previously dominant nickelcadmium (NiCad) and nickel-metal-hydride (NiMH) cells. Average open cell voltage is about $4.1 \mathrm{~V}$, diminishing to an average of about $3.5 \mathrm{~V}$ under normal load, compared to the $1.2 \mathrm{~V}$ per cell typical of NiCad or NiMH chemistry.

Sizing a battery pack for a particular SUAV design involves the selection of both capacity (size of cells measured in milliampere hours, or mAh) and voltage (the number of cells in series). The voltage required is determined by the motor being 
used because the motor's windings will be tailored to a particular voltage and power range. The capacity of the cell or battery is basically a measure of the amount of electricity the fully charged cell can deliver until it is discharge to a safe, specific cutoff voltage. Because a cell will slowly drop in voltage as it is discharged, it is important to know what safe cut-off voltages should be used to terminate the cell's discharge. LiPos are more sensitive to a minimum cell voltage than NiCad and NiMH. A minimum cut-off voltage of $3.0 \mathrm{~V}$ per cell is usually specified by LiPo cell manufacturers. If a cell is allowed to fall below this voltage, damage begins to occur, and the cell's useful life is reduced. This cut-off function is often accomplished by the motor electronic-speed-control (ESC) device.

In order to reach the desired voltage and capacity, individual cells of identical capacity can be combined into a battery by connecting them in series to add voltage and/ or in parallel to increase capacity. Due to the greater specific energy and power the LiPo cells were chosen for the proposed aircraft.

One peculiarity of batteries is that they are not consumed during flight. This means that, unlike normal fuel that is consumed and constantly reduces aircraft weight during the flight, battery fuel weight is fixed. From [26] it can be shown that for the case of battery-powered flight, the endurance of a given aircraft is proportional to:

$$
\frac{W_{B} / W_{1}}{\left(1+W_{B} / W_{1}\right)^{3 / 2}}
$$

Where $\mathrm{W}_{\mathrm{B}}$ is battery weight and $\mathrm{W}_{1}$ is aircraft weight less battery. Keeping $\mathrm{W}_{1}$ constant, and varying $\mathrm{W}_{\mathrm{B}}$, it follows that endurance is maximized when $\mathrm{W}_{\mathrm{B}}=2 \times \mathrm{W}_{1}$ , that is, when battery weight is equal to twice the empty weight of the aircraft, or is 2/3 of gross weight. This relationship is shown in Fig. 26 reproduced from [26]. 


\section{T/Tmax vs Wb/W1}

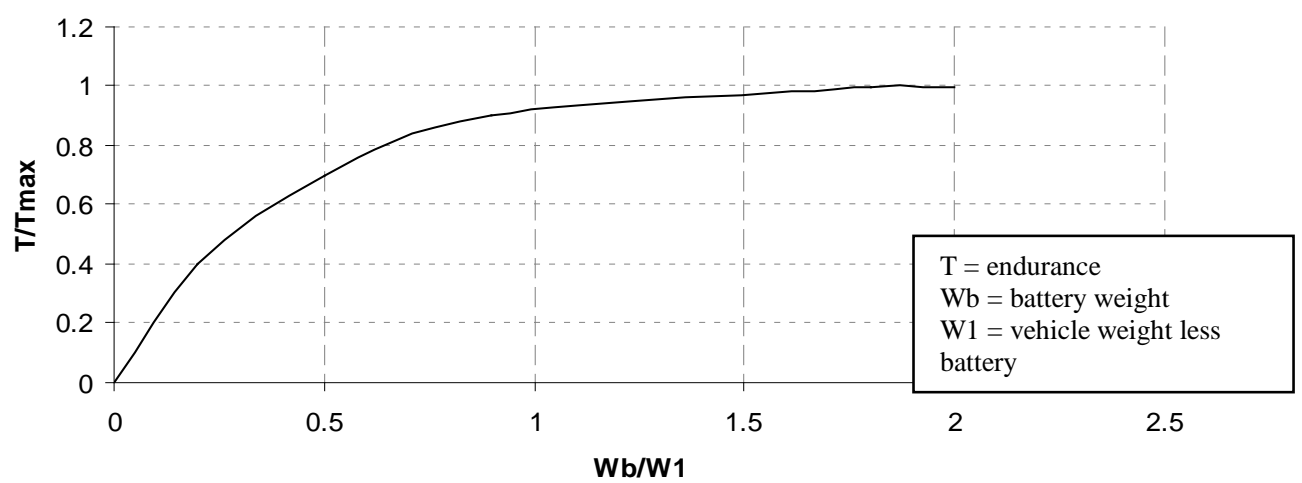

Fig.26 effect of battery weight on endurance partially reproduced from [26]: This graph shows the effect of battery weight on endurance. The maximum region is very flat and endurance is not reduced much as battery falls below $2 \times W_{1}$. Therefore the battery weight should be around $25 \%$ of the gross weight of the Small UAV. .

As it is shown in Fig. 26, the maximum region is very flat, and endurance is not reduced much as battery weight falls below $2 \times \mathrm{W}_{1}$. Therefore the conclusion is that if endurance and range are important SUAV objectives, a battery weight in the neighbourhood of at least $25 \%$ of the gross weight or more should be considered; below 25\%, the endurance potential declines more and more deeply. Therefore if the gross weight is $3.0 \mathrm{~kg}$, the battery weight should be around $0.75 \mathrm{~kg}$ at least.

\subsubsection{Electric Motor}

For a specific vehicle design, electric motor selection is determined primarily by power requirements and secondarily by considerations of weight, size, and efficiency. Among the bewildering variety of electric motors now available, there are three basic types suitable for SUAV applications showed in Fig. 27. 


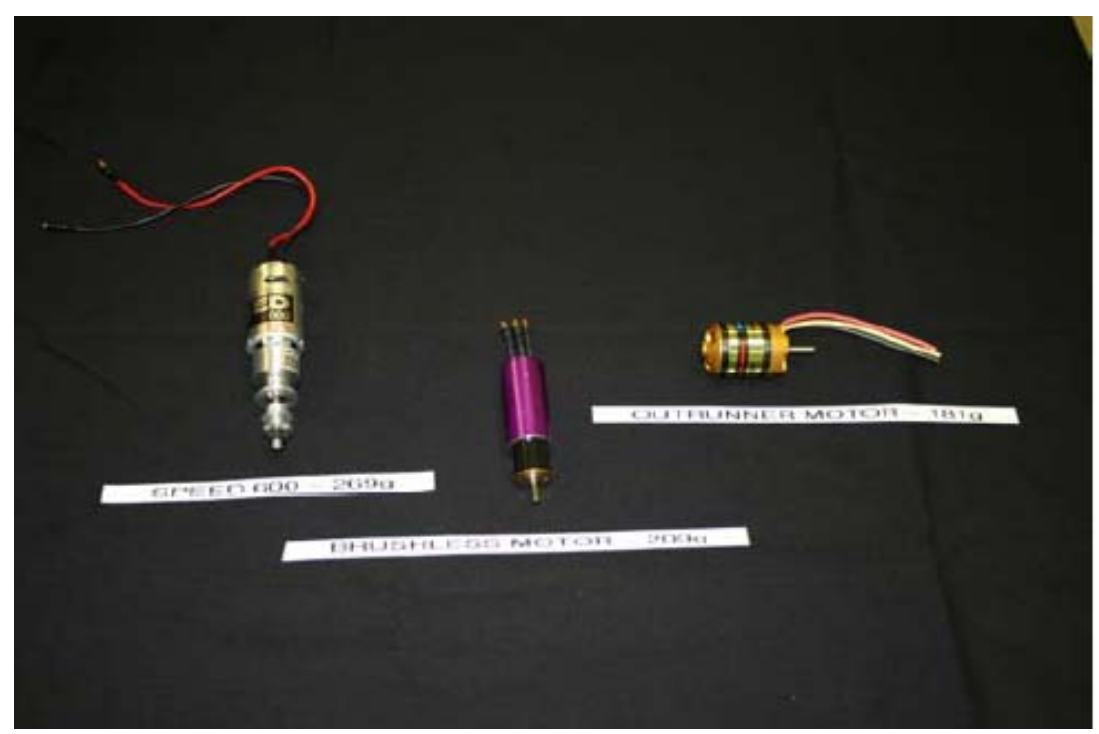

Fig. 27 Types of electric motors: There are 3 basic types of electric motors suitable for Small UAV applications and they are shown in this picture. On the left there is a traditional brushed motor. In the middle there is an inner brushless motor and on the right there is an outrunner brushless motor.

The motor on the left is a "traditional" brushed motor and gearbox circa 2001 that has a mass of $269 \mathrm{~g}$.

The middle motor is an inner brushless motor replacement, which has a mass of 209 g, a savings of 22.3\%. Brushless motors offer several advantages over brushed motors, including higher efficiency and reliability, reduced noise, longer lifetime (no brush erosion), elimination of ionizing sparks from the commutator, and overall reduction of electromagnetic interference.

The motor on the right is an "outrunner” brushless motor or external can motor which drives the propeller directly. This high torque motor eliminates the necessity for a gearbox thus providing a further weight savings of $13.3 \%$ over the inrunner brushless motor. Also seen is as a reduction in the number of moving parts, enhancing its reliability. Costs for these improved motors also seem to be decreasing as their utility becomes more widespread amongst mass-market users. One potential drawback to the outrunner motor appears in the form of integration. Since the majority of the 
external surface is rotating, there is no direct way to attach a heat sink to aid in cooling, as would be the case for the other motor types. This requires more consideration for cooling airflow and the associated impacts of that flow onto the overall system design.

Due to the potential advantages in performance and weight, the choice of an outrunner brushless motor was preferred for the proposed aircraft.

\subsubsection{Selecting motor/battery combination}

In order to choose the right combination of motor and battery that meets the requirements of the mission of the SUAV under study, the motor power required was estimated.

The power required for level, unaccelerated flight is given by the following equation:

$$
P_{R}=W\left(\frac{W}{S}\right)^{1 / 2}\left(\frac{C_{D}}{C_{L}^{3 / 2}}\right)\left(\frac{2}{\rho}\right)^{1 / 2} \frac{1}{\eta_{P}}
$$

A similar, but extended relationship has been developed by Hewitt Phillips in the form of watts of input power required per unit gross weight of the aircraft for various climb angles. For a given vehicle design with known aerodynamic and power system properties, the Phillips' relationship defines the required input power to the motor of an electrically powered vehicle as a function of wing loading and climb angle. It is given by:

$$
\frac{P_{B}}{W_{0}} \approx\left[\frac{1}{L / D}+\sin \phi\right] \times\left(\frac{1}{\eta_{p}}\right) \times\left(\frac{1}{\eta_{M}}\right) \times \sqrt{\frac{W_{0}}{S} \times \frac{2}{\rho C_{L}}}
$$


Where $P_{B}$ is the motor input power that the battery must supply, $\mathrm{W}_{0}$ is gross weight, $\mathrm{S}$ is wing area, $\mathrm{C}_{\mathrm{L}}$ is the lift coefficient, $\phi$ is the climb angle, $\eta_{p}$ is the propeller efficiency and $\eta_{M}$ is the motor efficiency.

Therefore with the information gathered in the previous sections,

$\mathrm{W}_{0}=3 \mathrm{Kg}$

$\mathrm{L} / \mathrm{D}=7.6$

$\eta_{p}=70 \%$

$\eta_{m}=80 \%$

$\mathrm{W} / \mathrm{S}=61 \mathrm{~N} / \mathrm{m}^{2}$

$\mathrm{C}_{\mathrm{L}}=0.25$

It is possible to compute the battery input power and motor output power required by the proposed SUAV for various climb angles. The results are plotted in Fig. 28.

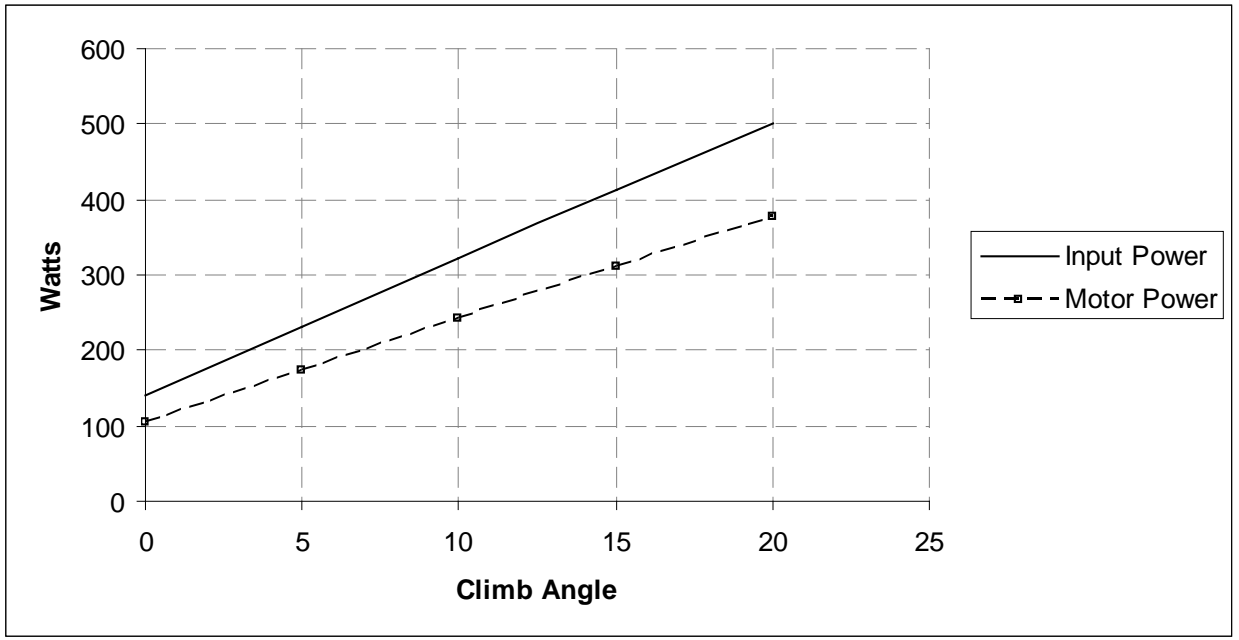

Fig.28 Power required vs. climb angle: This graphs shows the power required from the battery and from the motor vs. climb angle. It is noticed that for 0 climb angle, which means level flight, the power required from the battery is about $4.73 \mathrm{~W} / \mathrm{N}$. The motor output power at that input is $104.58 \mathrm{~W}$. At a climb angle of 10 degree, the power required is $242 \mathrm{~W}$ and the battery power is about $323 \mathrm{~W}$. 
For level flight the power required from the battery is about $4.73 \mathrm{~W} / \mathrm{N}$ or 139.44 W total. The motor output power at that input is about $104.58 \mathrm{~W}$ total.

At full power, the vehicle should be able to sustain a climb angle of about $10 \mathrm{deg}$, that corresponds to $242 \mathrm{~W}$. The battery power is about $323 \mathrm{~W}$.

On the basis of this consideration and on the basis of the gross weight of the proposed SUAV of $3 \mathrm{~kg}$, it was chosen the following Electric Motor, shown in Fig.29

\footnotetext{
Specifications

\# of Cells

RPM per Volt

Max. Efficiency

Voltage

Power output

Power input

No load current $/ 8 \mathrm{~V}$

Max. Loading

Internal resistance

Dimensions

Shaft Diameter

Weight with power wires

AXI4120/14
4-5 Li-Poly
12-16 Ni-XX
$660 \mathrm{RPM} / \mathrm{V}$
$85 \%$
$18.5 \mathrm{~V}$
$314 \mathrm{~V}$
$373 \mathrm{~V}$
$2 \mathrm{~A}$
$55 \mathrm{~A}$
$41 \mathrm{ohm}$
$49 \times 55 \mathrm{~mm}$
$6 \mathrm{~mm}$
$320 \mathrm{~g}$

AXI4120/14

12-16 Ni-XX

$660 \mathrm{RPM} / \mathrm{V}$

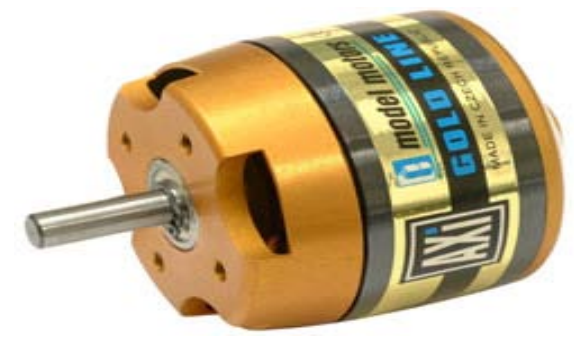

Recommended model weight $3000-4500 \mathrm{~g}$

Propeller range. Direct drive. $12-15 \times 6-8$ in

Fig.29 Electric motor chosen: This figure shows the electric motor chosen for the proposed aircraft. As it can be noticed the motor at full power has a out power of $314 \mathrm{~W}$ which is more than enough for the flight mission requirements for the proposed aircraft.
}

This motor, suitable [27] for airplane from $2000 \mathrm{~g}$ to $4000 \mathrm{~g}$ in weight, at full power has a out power of $314 \mathrm{~W}$ which allows to sustain the required $10^{\circ}$ climb angle (242 W). Therefore at maximum power the motor provides an output power margin of $72 \mathrm{~W}$ or $23 \%$ above the requirement. 
In order to keep a long motor life it was also decided to use a cooler for AXI 4120 series of outrunners.

For the controllers it was preferred to use Jeti 70 to 77 Amp Advance PLUS Opto brushless motor controllers, useful to set to 3.0 for the Lipo Battery, the low voltage cutoff (LVC). The weight is 35 grams.

A series of $5 \mathrm{Li}$-po batteries was selected in order to meet the voltage range of 18.5 $\mathrm{V}$ required by the selected motor.

At this point it is possible to estimate that the $18.5-\mathrm{V}$ battery must supply

- $\quad 139.44 \mathrm{~W}$ during level flight, that is $7.5 \mathrm{~A}$ at $18.5 \mathrm{~V}$

- $\quad 323 \mathrm{~W}$ during maximum power operation, that is $17.45 \mathrm{~A}$ at $18.5 \mathrm{~V}$

The nominal mission flight profile (Fig.14) is known. It is possible to estimate the expected times at maximum power (takeoff, climb out, and manoeuvre) and at cruise power (transit time):

- Takeoff and climb out and manoeuvres: if the average speed is:

$\mathrm{v}=15 \mathrm{~m} / \mathrm{s}$

The target operating altitude is:

$\mathrm{h}=150 \mathrm{~m}$

And the max climb angle is:

$\phi=10^{\circ}$

Then the distance to reach the target altitude is

$\mathrm{d}=\mathrm{h} / \phi=867 \mathrm{~m}$

therefore the time of takeoff and climb out is:

$\mathrm{t}=\mathrm{d} / \mathrm{v}=57 \mathrm{~s}$ 
Considering also a few manoeuvres to perform during the flight and a safety coefficient of 1.2 , the total time at maximum power is 3 minutes

- Cruise: the total cruise is estimated to be 57 minutes to reach the mission requirement of 1 hour of endurance.

The total battery capacity required is as follows:

Max power capacity $=3$ minutes $x 17.45 \mathrm{~A}=52.35 \mathrm{Am}$

Cruise capacity $=57$ minutes $x 7.5 \mathrm{~A}=427.5 \mathrm{Am}$

Total capacity $=1$ hour $=479.85$ Am $=7965.5$ mAh

Connecting batteries in parallel increases the total capacity. The following battery combination was selected (Table 3):

\begin{tabular}{|l|}
\hline $\begin{array}{c}\text { Thunder Power RC TP-8000-5S4P (5 cells in series, } \mathbf{4} \\
\text { in parallel) ProLite MS Military Spec LiPo: }\end{array}$ \\
\hline Voltage: $18.5 \mathrm{~V}$ \\
\hline Cells: 5S4P \\
\hline Capacity: 8000mAh \\
\hline Max Continuous Discharge: 16C \\
\hline Max Burst Discharge: $25 \mathrm{C}$ \\
\hline Max Continuous Current: 128A \\
\hline Max Burst Current: 200A \\
\hline Weight: 790g \\
\hline Dimensions: $29 \times 50$ x 325mm \\
\hline
\end{tabular}

Table 3 Selected battery combination: The table shows the selected battery combination: 5 batteries in series and 4 in parallel. The capacity of $8000 \mathrm{mAh}$ meets the endurance requirements and the weight equals to the $26 \%$ of the gross weight is in agreement with section 4.4.4.1

The capacity of $8000 \mathrm{mAh}$ of the selected battery meets the requirement of $7964.5 \mathrm{mAh}$ of total capacity necessary to perform the mission. It is interesting to see 
how the endurance of the proposed aircraft varies if the mission profile changes, keeping constant the total battery capacity. For this purpose a graph was drawn in which on the $\mathrm{x}$-axis there are the minutes at maximum power and on the $\mathrm{y}$ axis there are the minutes at cruise power. The more time the aircraft operates at maximum power the less endurance it has, as it was easy to predict. The graph in Fig. 30, in other words, shows all the possible mission profiles it is possible to perform once the total battery capacity has been chosen. The red dot represents the selected mission profile.

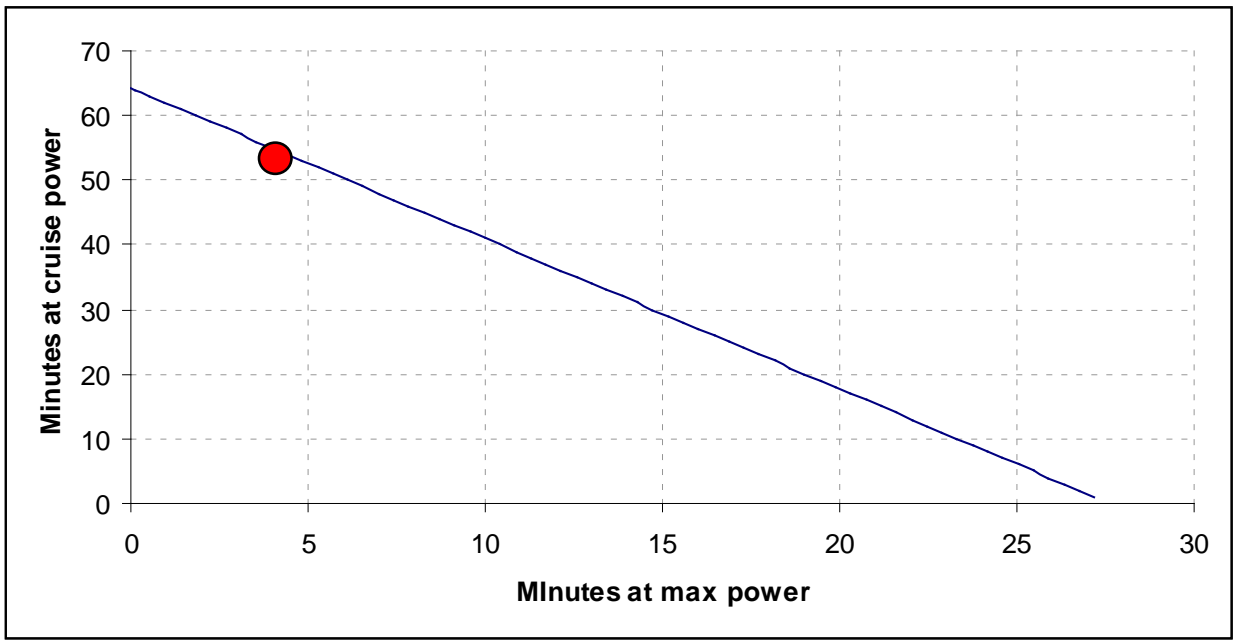

Fig.30 minutes at cruise power vs minutes at max power: This graph summarizes all the possible mission profiles it is possible to perform once the total battery capacity has been chosen. The red dot represents the selected mission profile.

Due the use of a twin boom, and due the hand-launched method, a small diameter propeller is preferred. Therefore the selected propeller was a 12 in dia x 6 in pitch as suggested by the electric motor technical sheet of Fig.29. 


\subsubsection{Empennage Sizing}

The empennage sizing is important to have a stable aircraft in flight. Tail sizing equations were taken from [7]. The quarter chord of the tail was placed 3 chords lengths after the aerodynamic centre of the wing.

The coefficients for the proposed Small UAV were found by researching comparatives UAVs, through the use of available pictures. The aircraft realized by the University of Dayton Aero Design Team for the SAE 2008 paper [28] was also taken into account as a reference, in order to check the order of magnitude was correct. The aspect ratio was chosen to minimize the dimensions of the aircraft.

The following equations were used to determine the areas of the vertical and horizontal tails:

$$
\begin{gathered}
S_{v t}=\frac{c_{v t} b S_{r e f, w i n g}}{L_{v t}} \\
S_{h t}=\frac{c_{h t} c S_{r e f, w i n g}}{L_{h t}}
\end{gathered}
$$

In this case we have the results summarized in Table 4.

\begin{tabular}{|c|c|c|c|c|c|c|}
\hline & $\mathrm{C}_{\mathrm{t}}$ & $\mathrm{L}_{\mathrm{t}}$ & $\begin{array}{c}\text { Surface Area Total } \\
\left(\mathrm{m}^{2}\right)\end{array}$ & $\mathrm{AR}$ & $\begin{array}{c}\text { Chord } \\
(\mathrm{m})\end{array}$ & $\begin{array}{c}\text { Span } \\
(\mathrm{m})\end{array}$ \\
\hline Vertical Tail & 0.0375 & $0.72 \mathrm{~m}$ & 0.049 & 1.1 & 0.19 & 0.21 \\
\hline Horizontal Tail & 0.675 & $0.72 \mathrm{~m}$ & 0.108 & 3 & 0.04 & 0.12 \\
\hline
\end{tabular}

Table 4 Vertical and horizontal tail specifications: This table shows the surfaces of the vertical and horizontal empennage. The A.R, chord and Span are also reported.

Further investigation is needed in the case of the use of a biplane. To compensate the increase in the moment in the yaw direction created by the wing endplates, and due to the fact that the weight of the endplates represents $1 / 4$ of the weight of the wings, the vertical tail volume coefficient was increased by twenty-five 
percent. The total Surface Area of the vertical tail becomes, in this case: $0.065 \mathrm{~m}^{2}$; the chord becomes $0.22 \mathrm{~m}$ and the span $0.24 \mathrm{~m}$.

\subsubsection{Other selected components}

4.4.6.1 Autopilot

The autopilot chosen was the Kestrel 2.2. At 16.65 grams (2” x 1.37” x .47”), Kestrel 2.2 is the smallest and lightest fully featured micro autopilot on the market ideal for surveillance and reconnaissance applications.

The system uses an external GPS unit for inertial navigation and wireless modems communications between the ground station and autopilot. The Kestrel Autopilot can guide mini- and micro-UAVs autonomously and/or receive dynamic user commands through the ground station, RC radio, and game pad controllers while providing live video feeds to the user. It uses three-axis rate gyros and accelerometers for attitude estimation, as well as differential and absolute air pressure sensors for airspeed and altitude measurement.

\subsubsection{Transmitter}

It was selected to use BlackWidow AV's collection of 2.4 Ghz transmitter/receiver to transmit the video signal. The video transmitter, from BlackWidow AV, is a $2.4 \mathrm{GHz}$ transmitter operating on $12 \mathrm{~V}$ DC. The transmitter is capable of transmitting audio as well as video, but for our purposes only video is necessary. The transmitter communicates directly to a standalone receiver that has a video output with a standard RCA connection. 
The video transmitter is connected to the camera only through a single video signal cable. Since the voltage for the transmitter is different than the camera, it does not share a common power supply. The video transmitter is powered by the same battery that powers the Kestrel autopilot.

\subsubsection{Camera setup}

It was selected the Procerus's camera setup, with forward and sideways looking camera options and varying lenses. The camera and gimbal setup is approximately 1.5 ” wide, 3.5 ” long, and 2.5 ” in height. The resolution of the camera is more than $450 \mathrm{TV}$ lines-or approximately $752 x 582$ pixels. The speed needed to focus on objects of interest will not be an issue with this gimbal, which can pan more than 360 degrees in less than a second. It can tilt a full 90 degrees, and can tilt approximately 80 degrees in less than half a second. The gimbal's components are made out of a carbon fibre composite. Together with the camera, the entire device is shielded inside a plastic enclosure. The entire combined mass is approximately 90 grams and it is shown in Fig. 31.

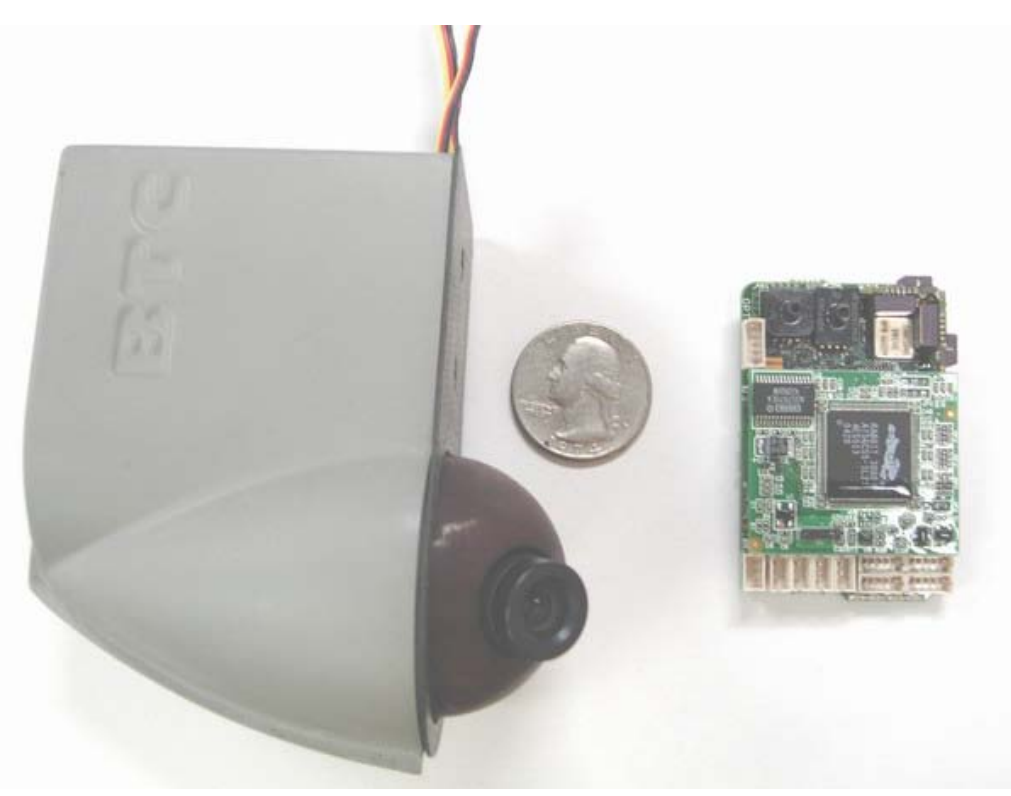


Fig. 31 Camera set up and transmitter: This figure shows the camera set up and the transmitter. The comparison with the coin demonstrates the small dimensions of the components in consideration.

\subsubsection{Weight build-up}

The estimation of the weight of a conceptual aircraft is a critical part of the design process. There are many levels of weights analysis. In this case using actual weight values performed the weight estimation of the aircraft. For parts that could not be weighed without first being built, such as the fuselage, wing, and tail assembly, estimates of the weight were based on data from past aircrafts and from material samples.

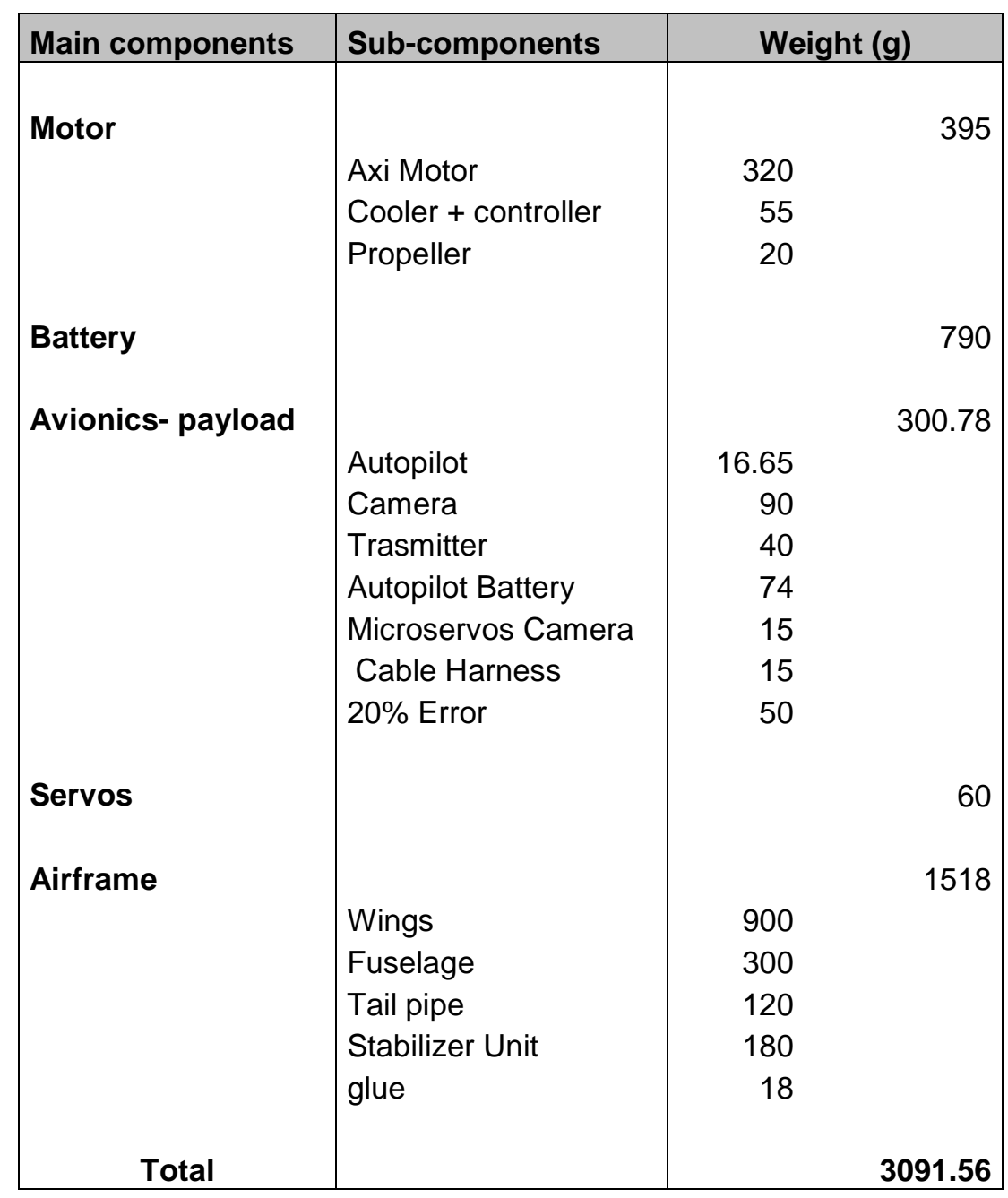


Table 5 Weight builds up: the different parts of the aircraft are reported with their weight and then added together. Four main different components have been individuated: The motor, the battery, the avionics and payload and the airframe. The total weight is $3091.6 \mathrm{~g}$.

The component weight breakdown of the aircraft is shown in Fig.31.

Weight breakdown
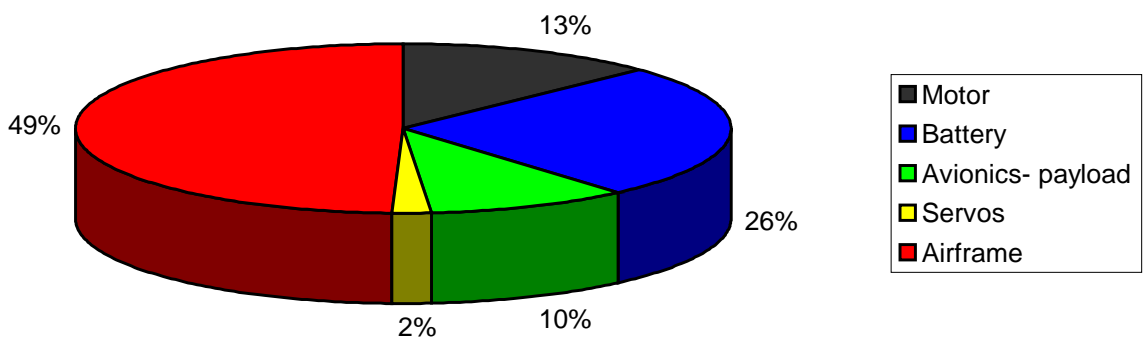

Fig. 32 Weight breakdown: This graph represents the component weight breakdown of the aircraft. The main weight is estimated to come from the airframe then the second biggest weight come from the battery. A battery weight in the neighbourhood of at least $25 \%$ of the gross weight or more should be considered [4.4.4.]; below 25\%, the endurance potential declines more and more deeply.

It can be noticed that as it was anticipated in section 4.4.4.1, a battery weight in the neighbourhood of at least $25 \%$ of the gross weight or more should be considered; below 25\%, the endurance potential declines more and more deeply. Therefore if the gross weight is $3.0 \mathrm{~kg}$, the battery weight should be around $0.75 \mathrm{~kg}$ at least.

It can be also noticed that the payload weight is less than the initial estimated payload. The causes of this difference lay probably in the fact that most of the comparative UAVs database presented the payload weight as the sum of the actual camera plus the avionic and cables needed. Another cause finds its root in the fast 
development of miniature cameras, which means that the new cameras are much lighter than that one used by the older UAVs.

On the other side it can be also noticed that the overall weight remains the same, or it is even increased of 100g. The reasons derive on one side from the bigger battery used able to accomplish the one hour target of endurance, and on the other side the use for the airframe of cheaper and weightier material in comparison with the very expensive and light material used by big companies like Aerovironment. Finally this represents only a rough estimation that must be revised in the next steps of the design process.

\subsubsection{Second Iteration}

Sine the overall weight, $3.091 \mathrm{~kg}$ results to be slightly increased from the initial estimation of $3 \mathrm{~kg}$, it is important to go again through the various steps of the design process and complete a second iteration in order to get a more accurate result.

\begin{tabular}{|c|c|}
\hline $\mathrm{W}_{\mathrm{TO}}$ & $3.1 \mathrm{Kg}$ \\
\hline $\mathrm{W}_{\mathrm{TO}} / \mathrm{S}$ & $61 \mathrm{~N} / \mathrm{m}^{2}$ \\
\hline $\mathrm{S}$ & $0.01 \mathrm{hp} / \mathrm{N}$ \\
\hline $\mathrm{hp} / \mathrm{N}$ & 8 \\
\hline $\mathrm{AR}$ & $2 \mathrm{~m}$ \\
\hline $\mathrm{b}$ & $0.25 \mathrm{~m}$ \\
\hline $\mathrm{c}$ & $0.067 \mathrm{~m}^{2}$ \\
\hline Vertical Tail Surface & \\
\hline Horizontal Tail Surface & $0.1125 \mathrm{~m}^{2}$ \\
\hline
\end{tabular}

Table 6 Design parameters: In this table the main characteristics of the aircraft after the change in weight are reported. Once the first iteration has been completed it is easy to insert just the new values and complete a second more refined iteration. 
In Table 6, the main characteristics of the aircraft are reported after the change in weight.

On the basis of the characteristics listed in table \#, it was possible to draw the 3 possible aircraft layouts that are shown in the next section.

\subsection{Layouts drawings}

Using the software Autodesk Inventor, 3 sketches of possible layouts have been drawn (Fig.33, 34, 35,36).

\section{$\checkmark$ Nonplanar wing configuration (Fig.33):}

For reasons that will be explained later in the aerodynamic analysis chapter, there is not stagger and the gap is equal to 1 chord. As it can be noticed it was chosen a pusher propeller: a propeller in front of the fuselage would have disturbed the camera from working and a propeller attached in front of the wing might have interfered with the fuselage. Due to the use of a pusher propeller, a twin boom tail was selected. 


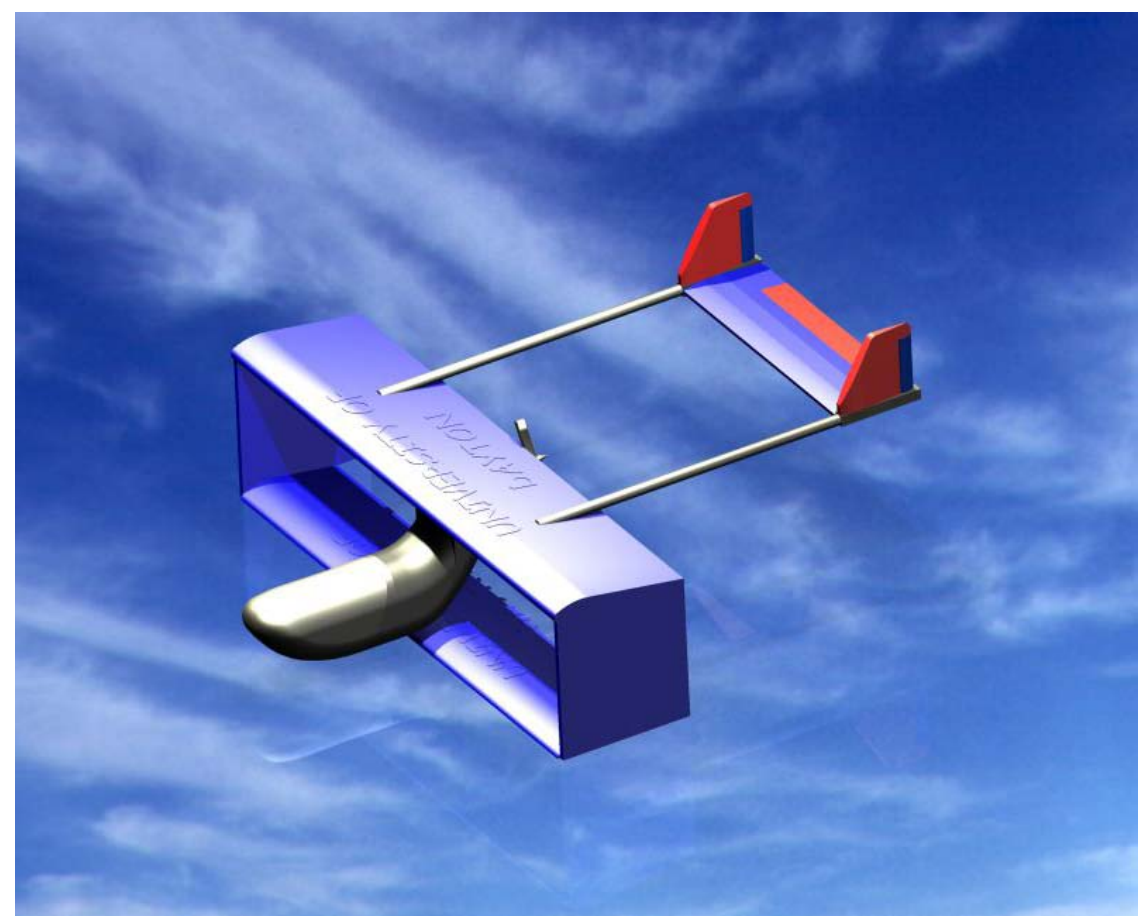

Fig.33 Layout 1: This image represents one possible layout for the proposed small UAV. The main characteristic of this layout is the nonplanar wing configuration. This drawing was realized using the software Autodesk Inventor 2008. The final rendering was realized using Inventor Studio, an application of the same software.

\section{$\checkmark$ Standard monoplane configuration (Fig.34):}

It is always interesting to compare the new possible layouts with the standard monoplane configuration to understand the potential advantages and disadvantages. In this case, it was chosen a pusher propeller: a propeller in front of the fuselage would have disturbed the camera from working and a propeller attached in front of the wing might have interfered with the fuselage. In order to avoid aerodynamic interferences between the wing and the tail, the horizontal tail was preferred to be at the bottom of the tail. The Monoplane configuration has the same Aspect Ratio of the nonplanar configuration analyzed in Fig.33. 


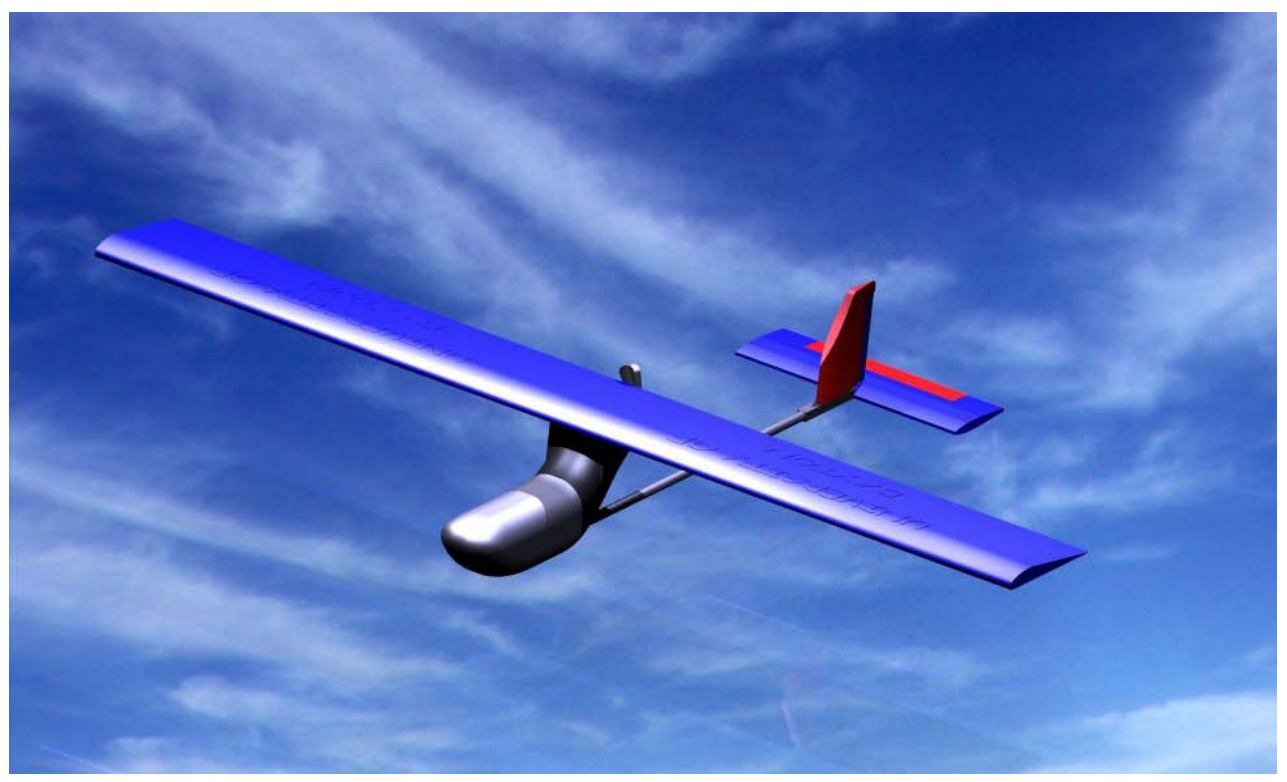

Fig.34 Layout 2:This image represents one possible layout for the proposed small UAV. This layout presents a standard monoplane configuration. This drawing was realized using the software Autodesk Inventor 2008. The final rendering was realized using Inventor Studio, an application of the same software.

\section{$\checkmark \quad$ Standard biplane configuration (Fig.35):}

For the standard biplane configuration it was chosen a pusher propeller: a propeller in front of the fuselage would have disturbed the camera from working and a propeller attached in front of the wing might have interfered with the fuselage. Due to the use of a pusher propeller, a twin boom tail was selected. The biplane configuration's Aspect Ratio is the same of the Fig. 33 and 34. The configuration described in Fig. 33 and the configurations described in Fig. 35 are very similar. The main difference is the use of endplates. In the next chapter it will be analyzed from a structural and aerodynamic point of view if using the configuration of Fig. 33 can bring any benefits with respect to the standard monoplane (Fig. 34) or with respect to the standard biplane (Fig. 35) 


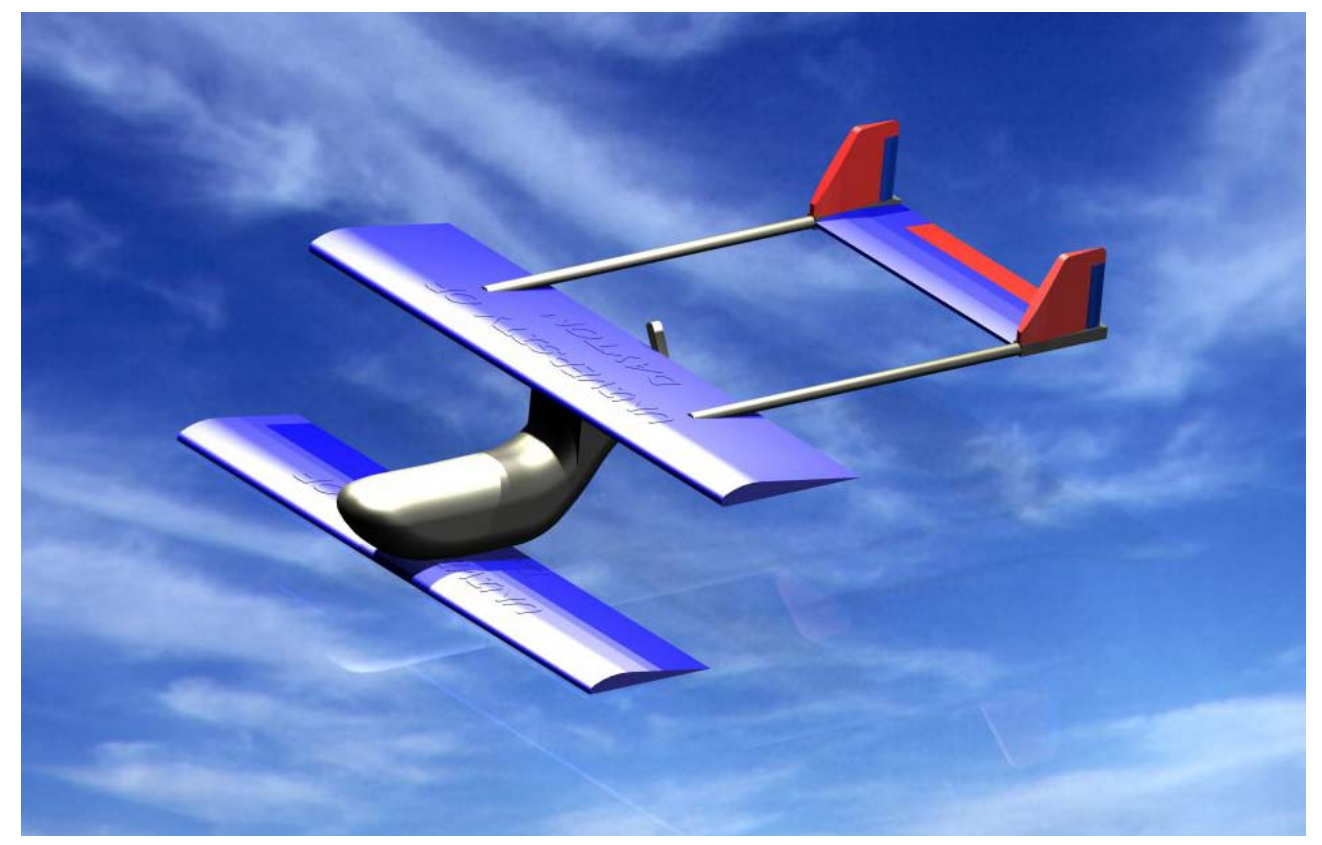

Fig.35 Layout 3: This image represents one possible layout for the proposed small UAV. The main characteristic of this layout is the standard biplane configuration. This drawing was realized using the software Autodesk Inventor 2008. The final rendering was realized using Inventor Studio, an application of the same software. 

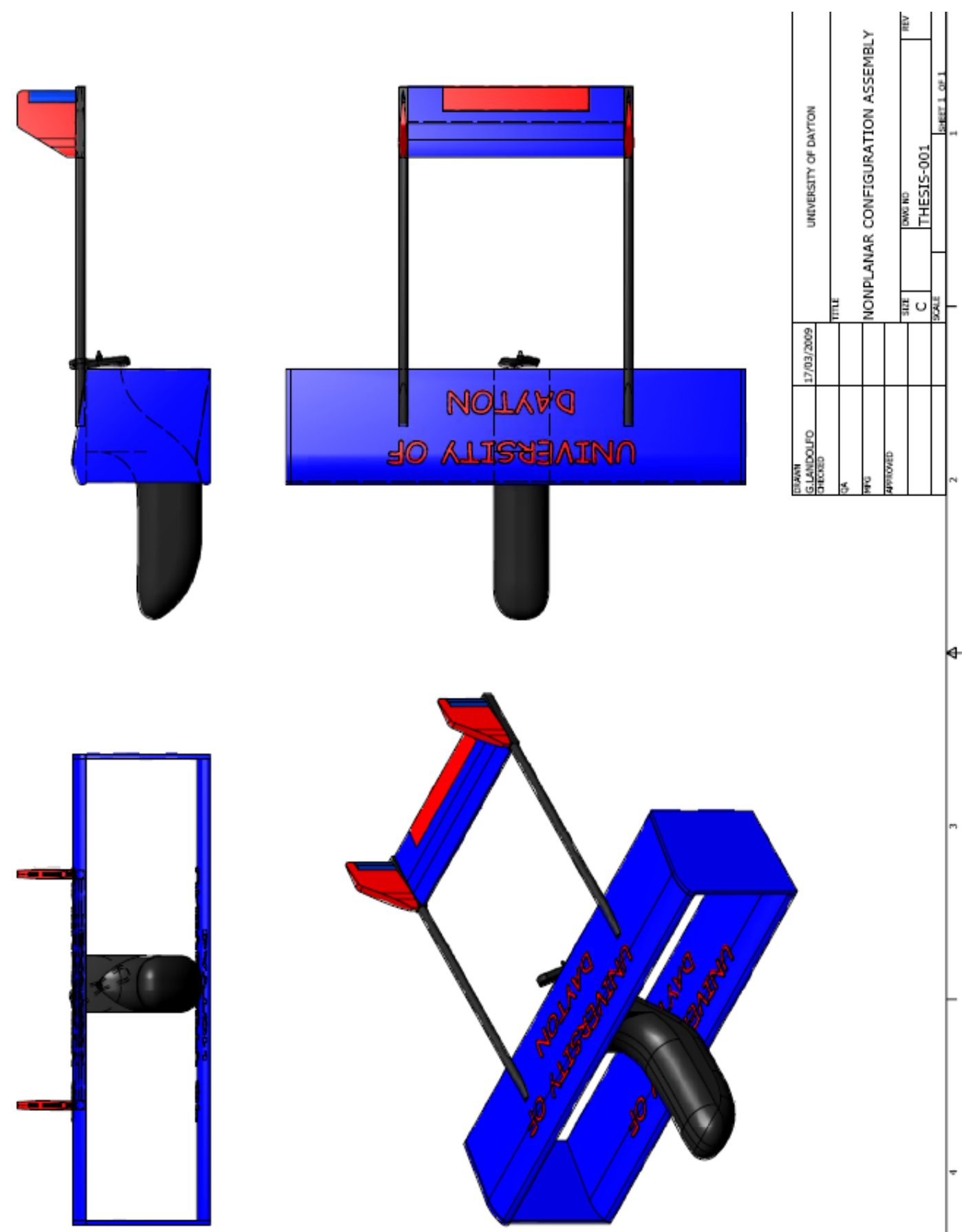

Fig.36 3 views of configuration 1: This figure shows the 3 views of the configuration of Fig. 33, that is a biplane with endplates. There is not Stagger and the Gap is equal to 1 chord. The 3 views show in more detail the configuration. 


\section{Chapter V}

\section{Structural Analysis}

\subsection{Aircraft loads}

Before the structure of the wing can be designed, we need to determine the loads that will be imposed on the aircraft's wing. This section deals with the general issue of aircraft loads and how they are predicted in the early stages of the design process.

\subsubsection{Maneuver Loads}

Each part of the aircraft is subject to many different loads. In the final design of an aircraft structure, one might examine tens of thousands of loading conditions of which several hundred may be critical for some part of the airplane.

The problem is simplified under the assumption of considering only the air loads, loads exerted onto the structure during flight by the manoeuvres carried out by the aircraft or by wind gusts. In particular the loads generated by the lift and the drag on the main wing will be analyzed. The crash loads, even though significantly for this class of aircraft, will be neglected in this study.

The largest load the aircraft is actually expected to encounter is called the limit or applied load. To provide a margin of safety, the aircraft structure is always 
designed to withstand a higher load than the limit load. The highest load the structure is designed to withstand without breaking is the "design" or "ultimate", load. The "factor of safety" is the multiplier used on limit load to determine the design load. In this case, since there are no specific requirements for the factor of safety, 1.5 will be used as the factor of safety, which is common in aeronautics.

The structural strength required of the airplane components is determined by the design maximum load factor specified for the airplane and varies with the function of the airplane’s mission.

Aircraft load factor, $\mathrm{n}$, expresses the manoeuvring of an aircraft as a multiple of the standard acceleration due to gravity and it is defined as $n=L / W$ with $n>0$ denoting wings pulled up and $n<0$ denoting wings pulled down. A load factor $n=1$ denotes steady level flight with $L=W$. Load factors different from $n=1$ are caused by manoeuvres such as turns, dives, climbs, etc.

Considering the mission profile of the aircraft studied, a Small UAV, the maximum load factor is related to the maximum level turn bank angle (70 deg) the airplane can sustain without damage. In fact in this case the equations of motion become, as shown in Fig.37.

$$
\begin{aligned}
& T-D=0 \\
& L \sin \varphi=\frac{W}{g} V \dot{\psi} \equiv \frac{W}{g} \frac{V^{2}}{r} \\
& L \cos \varphi-W=0
\end{aligned}
$$

From the last one

$$
n=\frac{L}{W}=\frac{1}{\cos \varphi}
$$

If we assume a maximum bank angle of $\varphi=70^{\circ}$ then

$$
n_{\max }^{+} \approx 1.6
$$


and using 1.5 as a safety factor

$n_{\text {ultimate }}^{+} \approx 2.4$
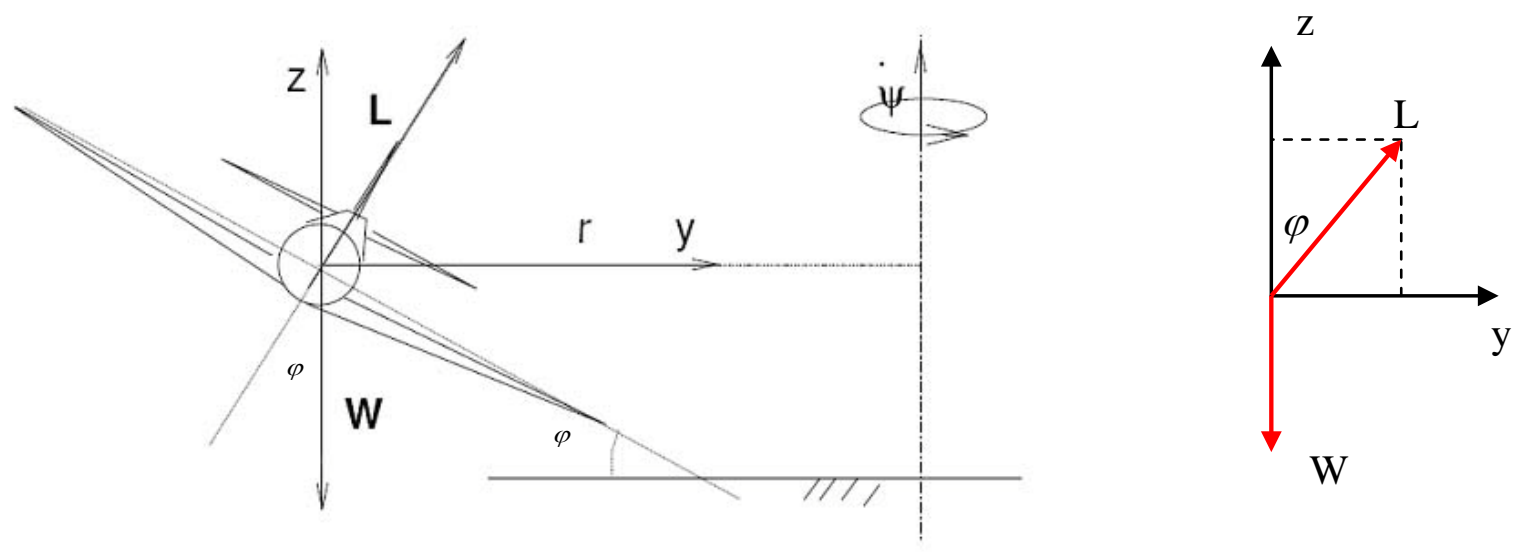

Fig.37: Aircraft's forces in turn. From the equilibrium of the forces along the z-axis, $L \cos \varphi-W=0$, and therefore $n=\frac{L}{W}=\frac{1}{\cos \varphi}$. Giving a value of $50^{\circ}$ to the maximum bank angle, and a safety factor of 1.5, the positive load factor becomes 1.6. In the same way it is possible to calculate the negative load factor, which results to be 1.8 .

The negative load factor is assumed to be

$n_{\text {Max }}{ }^{-}=1.2$

And so using 1.5 as a safety factor

$n_{\text {ultimate }}{ }^{-} \approx 1.8$

The next section introduces a very useful tool that clarifies the manoeuvre loading.

\subsubsection{V-n Diagram}

The V-n diagram (Fig. 38) illustrates the variation in load factor, just introduced, with airspeed for manoeuvres. In other words it is this aircraft operational envelope that ensures design loads are not exceeded. 
The aircraft speeds are in "equivalent” airspeed $V_{e}$, where

$$
V_{e}=\sqrt{\rho / \rho_{S L}}\left(V_{\text {actual }}\right)
$$

The lower limit on our V-n diagram is given by the lift line or $C_{L_{\operatorname{Max}}}$ boundary, which is calculated taking into account that in any manoeuvre the maximum lift that can be generated is $\mathrm{L}_{\max }=\mathrm{n}_{\max } \mathrm{W}$, which means that

$n_{\max }=\frac{C_{L, \max } q}{(W / S)}=\frac{C_{L, \max } \rho_{s . l .}}{2(W / S)} V_{E}^{2}$

So the lift line represents the stall speed for any load factor. In a conventional aircraft flight to the left of the line is not possible because the wing will stall first. Therefore the point A of the diagram reported in Fig.39 corresponds to the slowest speed at which the maximum load factor can be reached without stalling.

The diagram was prepared using a $C_{L \max }$ of 1.3 , a minimum $C_{L \min }$ of -0.4 determined from the airfoil section data, a wing loading of $61 \mathrm{~N} / \mathrm{m}^{2}$ from Chapter IV, a maximum load factor of 1.6 and -1.2 and a factor of safety of 1.5 as specified before.

The other end of the diagram is the maximum allowable speed. If we refer to the FAR 23 [29], the dive speed is required to be:

$$
V_{D} \geq 1.25 V_{C}
$$

Also as a first approximation the loiter speed is assumed as $70 \%$ of the cruise speed. But the loiter speed of this aircraft is a variable controlled by the sensor payload because images become blurry at fast speeds. So camera parameters constrain loiter speed as reported in the next plot shown in Fig. 38, which represents a regression created using a wide variety of micro video cameras on market today. 


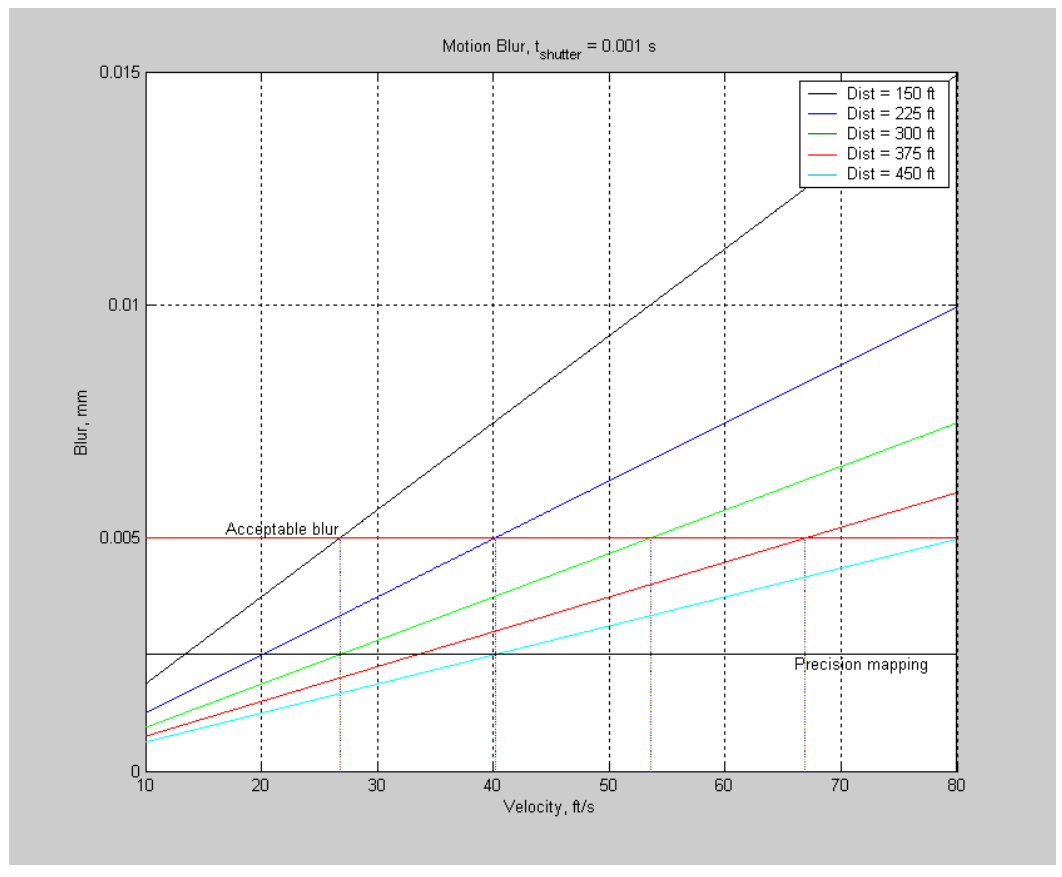

Fig. 38 level of blur vs. velocity: The loiter speed is a variable controlled by the sensor payload because images become blurry at fast speeds. The graph shows for a wide variety of micro video cameras on market today, the maximum speed allowable before images become blurry (reproduced from [30]).

Therefore in order to have an acceptable blur at a distance of 100 meters, the loiter speed is assumed to be $15 \mathrm{~m} / \mathrm{s}$. This value is considered reasonable when taken in context with the loiter speeds of other small UAVs with the same mission requirements in the historical database (Table 1).

In this case the cruise speed is around $19 \mathrm{~m} / \mathrm{s}$ and so finally

$$
V_{D} \geq 1.25 V_{C} \geq 23.5 \mathrm{~m} / \mathrm{s}
$$

In order to have a safety margin of $25 \mathrm{~m} / \mathrm{s}$ a dive speed is assumed. Using the dive speed plus the structural limits and equation [1] for the lift line, the V-n diagram was constructed. 


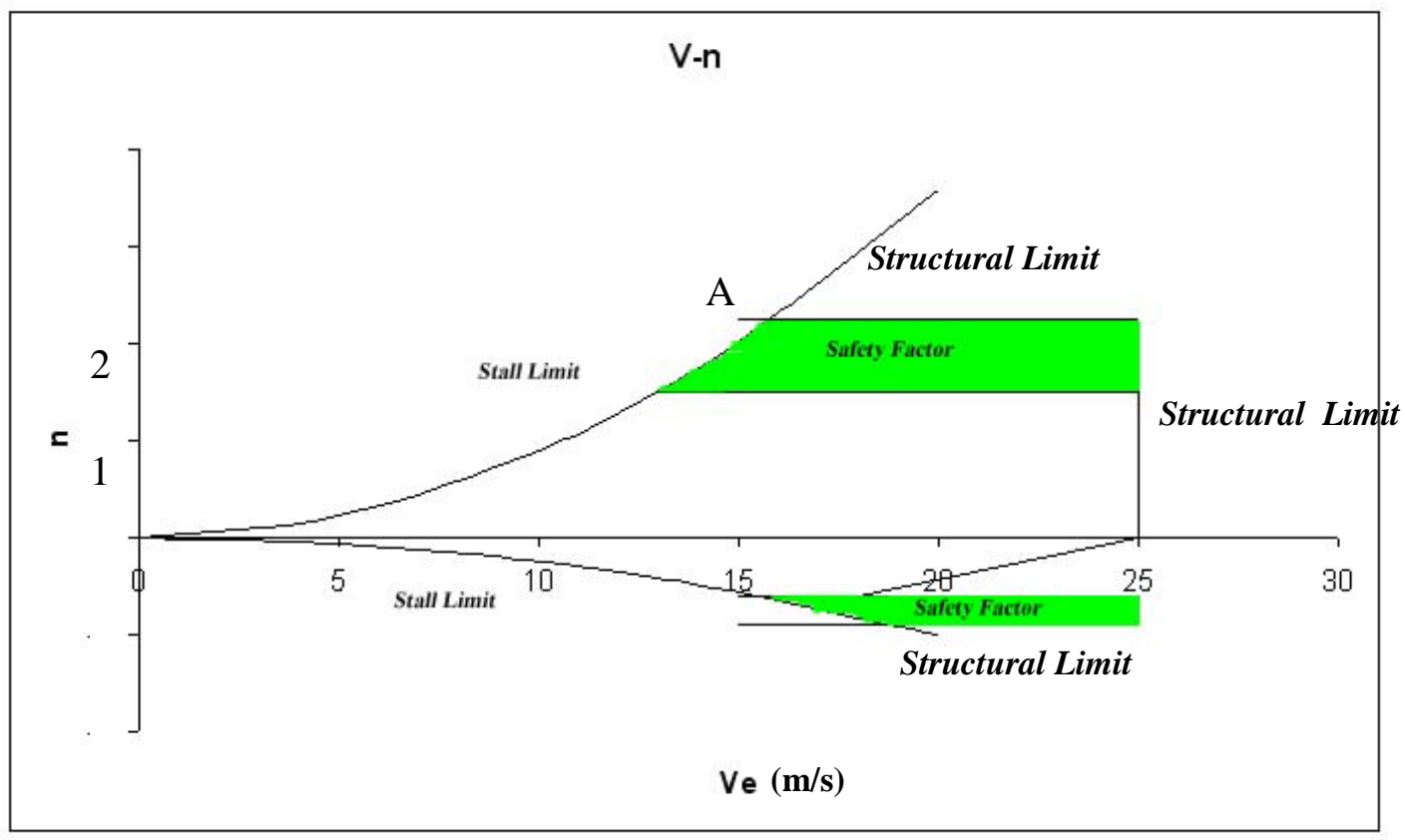

Fig.39: V-n diagram for a Small UAV. The V-n diagram shows the variation in load factor with airspeed for manoeuvres. In other words it is this aircraft operational envelope that ensures design loads are not exceeded. This diagram describes that at load factors higher than 3 or at speeds higher than $25 \mathrm{~m} / \mathrm{s}$, structural failure could occur

This diagram describes that at load factors higher than 3 or at speeds higher than $25 \mathrm{~m} / \mathrm{s}$, structural failure could occur. At speeds outside the curves on the left side flight is not possible because the airplane would be stalled.

\subsubsection{Gust Loads}

Since the UAV under study is small and has a limited maximum speed, it reacts easily to the force of the wind. Thus loads associated with vertical gusts must also be evaluated over the range of speeds (Fig.40). In some cases these loads can exceed the manoeuvre loads. 


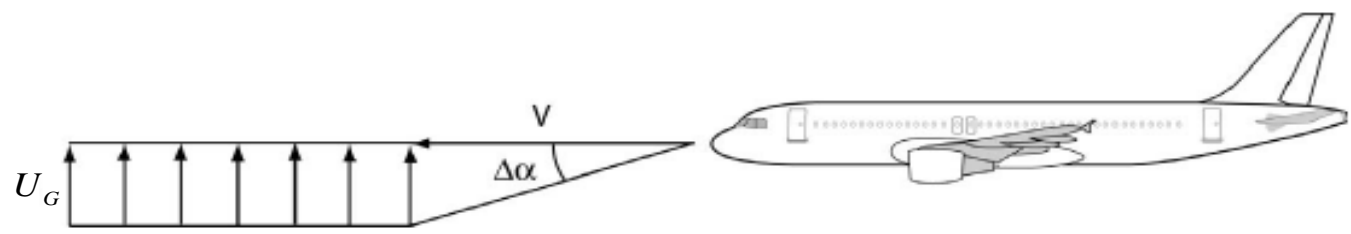

Fig. 40: Scheme of vertical gusts: a small UAV reacts easily to the force of the wind, therefore the loads associated with vertical gusts must also be evaluated over the range of speeds. $U_{G}$ is the speed of the gusts, $V$ represents the speed of the airplane and $\Delta \alpha$ represents the change in angle of attack due to the gusts.

The gust loads for an aircraft are determined by first estimating the change in angle of attack upon entering a sharp-edged gust:

$$
\Delta \alpha=\tan ^{-1} \frac{U_{G}}{V} \approx \frac{U_{G}}{V}
$$

The change in lift as a result of this change in angle of attack is estimated as:

$$
\Delta L=\frac{1}{2} \rho V^{2} S\left(C_{L_{\alpha}} \Delta \alpha\right)=\frac{1}{2} \rho V S C_{L \alpha} U_{G}
$$

The above equation does not include alleviation effects resulting from real unsteady aerodynamics; in reality, the lift does not change instantaneously when an airplane enters a gust, nor is a gust ever truly sharp-edged. Therefore, the gust velocity is tempered by a "gust alleviation factor," and

$\mathrm{U}_{\mathrm{G}}=\mathrm{K}_{\mathrm{G}} \mathrm{U}_{\mathrm{D}}$, where:

$$
\begin{aligned}
K_{G}= & \left\{\begin{array}{l}
\frac{0.88 \mu}{5.3+\mu} \rightarrow \text { Subsonic } \\
\frac{\mu^{1.03}}{6.95+\mu^{1.03}} \rightarrow \text { Supersonic }
\end{array}\right. \\
\mu & =\frac{2(W / S)}{\rho g c C_{L \alpha}} \rightarrow \text { mass_ratio }
\end{aligned}
$$

To calculate the change in load factor, $\mathrm{n}=\mathrm{L} / \mathrm{W}$, and 
$n=\frac{L \pm \Delta L}{W}=1 \pm \frac{\rho V C_{L \alpha} K_{g} U_{d}}{2(W / S)}$

The gust load factors as calculated with equation [2] and using the appropriate $U_{d}$ can then be plotted on a V-n diagram.

For the UAV under study it is found

$\mu=5.65$

$K_{g}=0.45$

and under the assumptions of using the $U_{D}$ reported in Table 7,

\begin{tabular}{|c|c|}
\hline $\mathrm{V}$ & $U_{D}(\mathrm{~m} / \mathrm{s})$ \\
\hline Vgust & 7 \\
\hline Vcruise & 6 \\
\hline Vdive & 3 \\
\hline
\end{tabular}

Table 7: $U_{D}$ for three characteristic conditions: gust, cruise and dive. Using these estimated speed it is possible to create the gust diagram reported in Fig. 37

For stall speed, V=14 m/s

$$
n=\frac{L \pm \Delta L}{W}=1 \pm \frac{\rho V C_{L \alpha} K_{g} U_{d}}{2(W / S)} \rightarrow\left\{\begin{array}{l}
n^{+}=3.65 \\
n^{-}=-1.65
\end{array}\right.
$$

For cruise speed, V=18 m/s

$$
n=\frac{L \pm \Delta L}{W}=1 \pm \frac{\rho V C_{L \alpha} K_{g} U_{d}}{2(W / S)} \rightarrow\left\{\begin{array}{l}
n^{+}=3.92 \\
n^{-}=-1.92
\end{array}\right.
$$

For dive speed, $\mathrm{V}=25 \mathrm{~m} / \mathrm{s}$ 
$n=\frac{L \pm \Delta L}{W}=1 \pm \frac{\rho V C_{L \alpha} K_{g} U_{d}}{2(W / S)} \rightarrow\left\{\begin{array}{l}n^{+}=3.02 \\ n^{-}=-1.02\end{array}\right.$

Using the aforementioned load factors the gust diagram can be constructed as seen in Fig. 41.

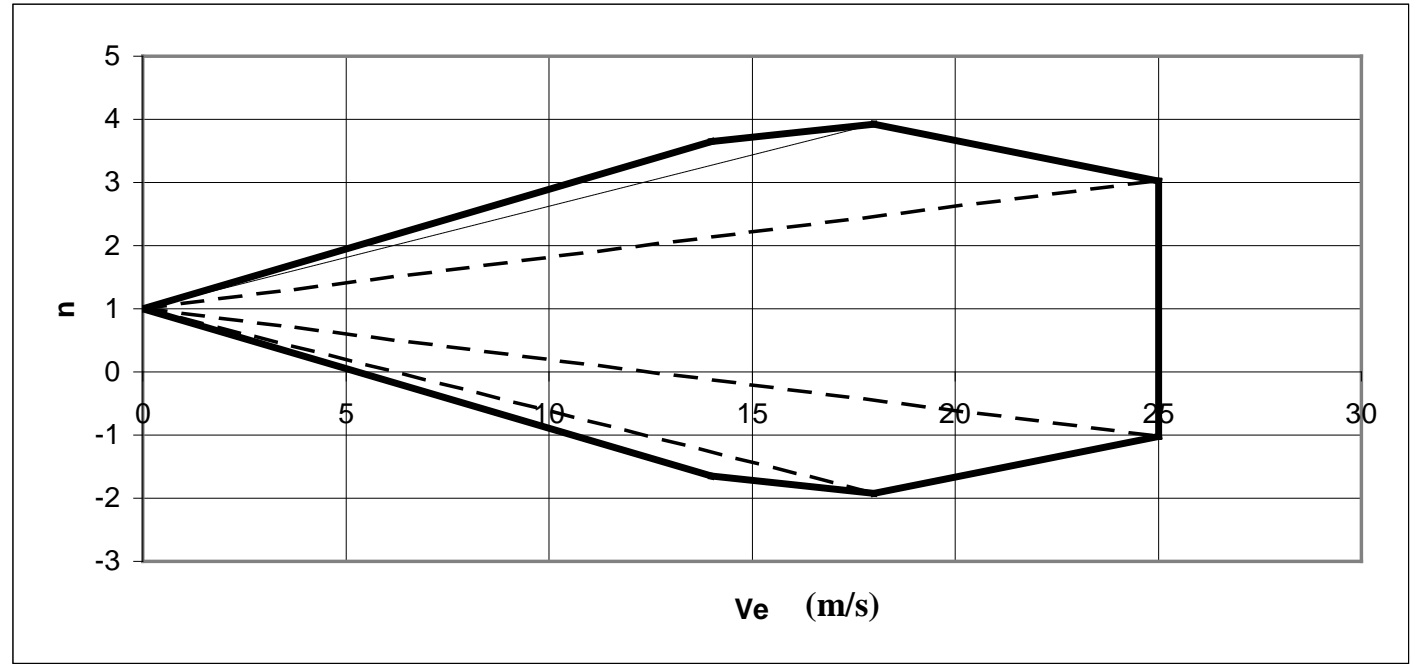

Fig. 41: gust diagram for a Small UAV. For each of the characteristic speeds, stall, cruise and dive speed, the load factor was calculated. It can be noticed that the highest load factor is reached for the cruise speed and is 3.92 .

\subsubsection{Wing structure}

Testing of wing structures had been previously conducted at UD [28], and it was shown that foam core carbon fibre composites have superior strength to weight ratios compared to balsa ribbed wings that utilize a carbon fibre tube spar, due to failure of the balsa ribs at lower loading, especially when normalized by the structural weight of each type of wing. The results for this testing are shown in Table 8 below: 


\begin{tabular}{|c|c|c|c|}
\hline $\begin{array}{c}\text { Properties Foam } \\
\text { Composite }\end{array}$ & Value & $\begin{array}{c}\text { Properties Balsa } \\
\text { Composite }\end{array}$ & Value \\
\hline$\sigma_{\text {tension }}$ & $1034 \mathrm{MPa}$ & $\sigma_{\text {tension }}$ & $275.8 \mathrm{MPa}$ \\
\hline$\sigma_{\text {compression }}$ & $344.73 \mathrm{MPa}$ & $\sigma_{\text {compression }}$ & $275.8 \mathrm{MPa}$ \\
\hline $\begin{array}{c}\text { Composite Modulus of } \\
\text { Elasticity }\end{array}$ & $10342 \mathrm{MPa}$ & $\begin{array}{l}\text { Composite Modulus of } \\
\text { Elasticity }\end{array}$ & $8963 \mathrm{MPa}$ \\
\hline $\begin{array}{l}\text { Carbon Fiber } \\
\text { Thickness, } \mathrm{t}\end{array}$ & $0.15 \mathrm{~mm}$ & Spar Tube Thickness & $1.59 \mathrm{~mm}$ \\
\hline $\begin{array}{l}\text { Strength to Weight } \\
\text { (lbs held per lb) }\end{array}$ & 250 & $\begin{array}{l}\text { Strength to Weight } \\
\text { (lbs held per lb) }\end{array}$ & 140 \\
\hline
\end{tabular}

Table 8: properties of foam and balsa: The foam core carbon fibre composites have superior strength to weight ratios compared to balsa ribbed wings that utilize a carbon fibre tube spar, due to failure of the balsa ribs at lower loading, especially when normalized by the structural weight of each type of wing.

The semi span wing structures are exposed to torsion and bending stress as explained in the next section and a possible wing box is shown in Fig. 42.

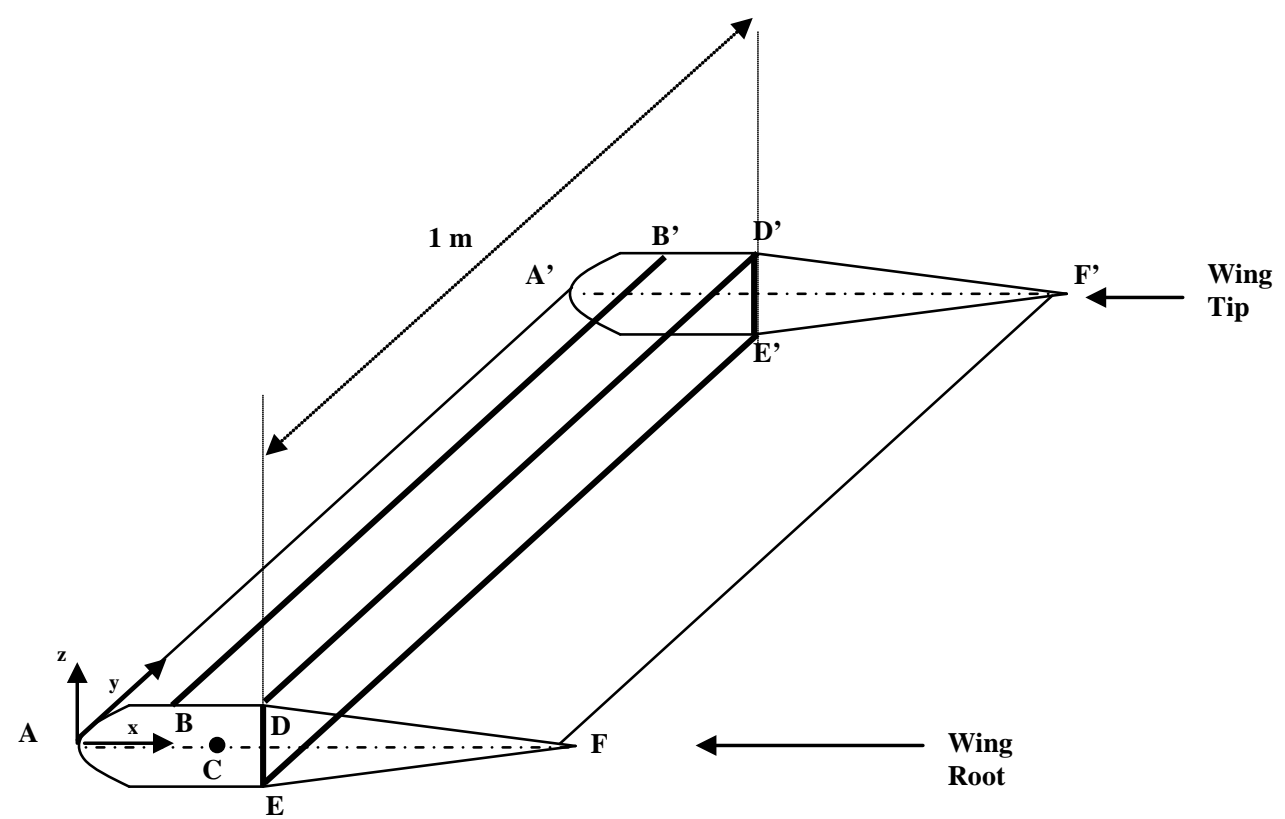

Fig. 42: example of wing box structure. Several elements can be noticed: 3 carbon fibre spar caps BB', DD', EE', 1 spar DD'EE' in carbon fibre, Foam core, Fibreglass skin.

From Fig. 42 it is possible to recognize the following structural components:

- 3 carbon fibre spar caps BB', DD', EE' 
- 1 spar DD’EE’ in carbon fibre

- Foam core

- Fibreglass skin

Using the physical dimensions calculated in Chapter IV

- $\mathrm{S}=0.5 \mathrm{~m}^{2}$

- $\quad \mathrm{AR}=8$

- $\quad b=2 m$ and therefore $b / 2=a=1 m$

- $\quad c=0.25 \mathrm{~m}$

- $\quad B D=0.06 \mathrm{~m} ; \mathrm{DE}=0.02 \mathrm{~m}$

The centre of pressure c is assumed to be at the quarter chord where the lift is applied.

\subsubsection{Load distribution}

Now that the V-n diagram is complete, the actual loads and load distributions on the lifting surfaces can be determined. An elliptical shape span wise lift distribution is assumed and can be represented by the following equation plotted in Fig.43.

$$
L(y)=L_{0} \sqrt{1-\left(\frac{y}{a}\right)^{2}}
$$

Where $\mathrm{L}_{0}$ is the force per unit length at the root of the wing and $a$ is the length of the semi span wing, in this case $1 \mathrm{~m}$.

As previously introduced, since the total weight of the Small UAV is $3.1 \mathrm{Kg}$ and the load factor is $n=2.4$, the total lift on the semi span wing is $L=n W=73 \mathrm{~N}$

But $\mathrm{L}$ is also

$$
L=\int_{0}^{L} L(y)=\int_{0}^{L} L_{0} \sqrt{1-\left(\frac{y}{a}\right)^{2}} d y=L_{0} \int_{0}^{L} \sqrt{1-\left(\frac{y}{a}\right)^{2}} d y
$$


and in this way the unknown $\mathrm{L}_{0}$ can be calculated:

$$
L_{0}=\frac{L}{\int_{0}^{L} \sqrt{1-\left(\frac{y}{a}\right)^{2}} d y}=93 \mathrm{~N} / \mathrm{m}
$$

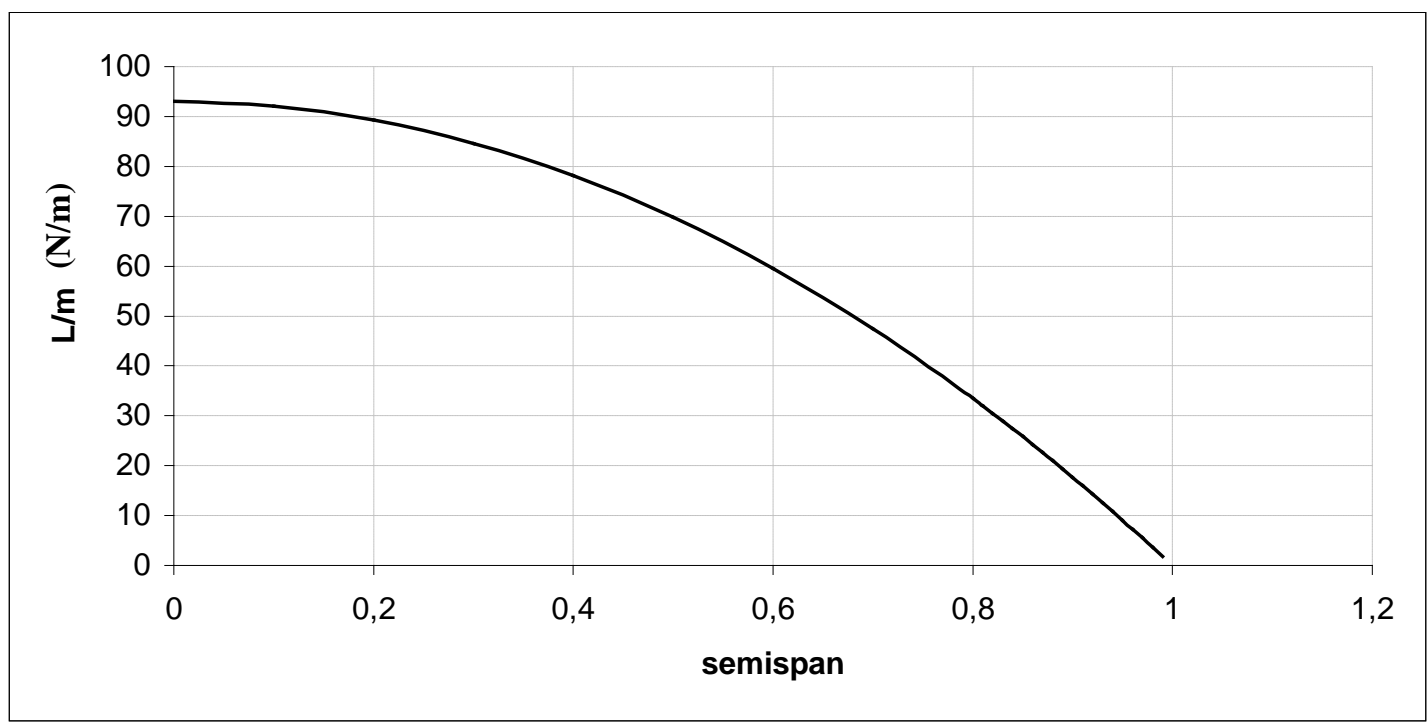

Fig. 43: Elliptical distribution of the lift along the semi span. $L(y)=L_{0} \sqrt{1-\left(\frac{y}{a}\right)^{2}}$ is the equation in order to obtain the elliptical distribution where $L_{0}$ is the force per unit length at the root of the wing and $a$ is the length of the semi span wing, in this case $1 \mathrm{~m}$.

In order to simplify the calculations, and as a hand calculations exercise , it is possible as a first approximation to approximate the elliptical distribution of the lift with a constant distribution from the root to the $2 / 3$ of the semi span and then a linear distribution from the $2 / 3$ of the span to the tip of the wing as shown in Fig. 44 and specified below:

$$
\left\{\begin{array}{l}
L(x)=L_{0} \rightarrow y \leq \frac{2 a}{3} \\
L(x)=3 L_{0}\left(1-\frac{y}{a}\right) \rightarrow \frac{2 a}{3} \leq y \leq a
\end{array}\right.
$$




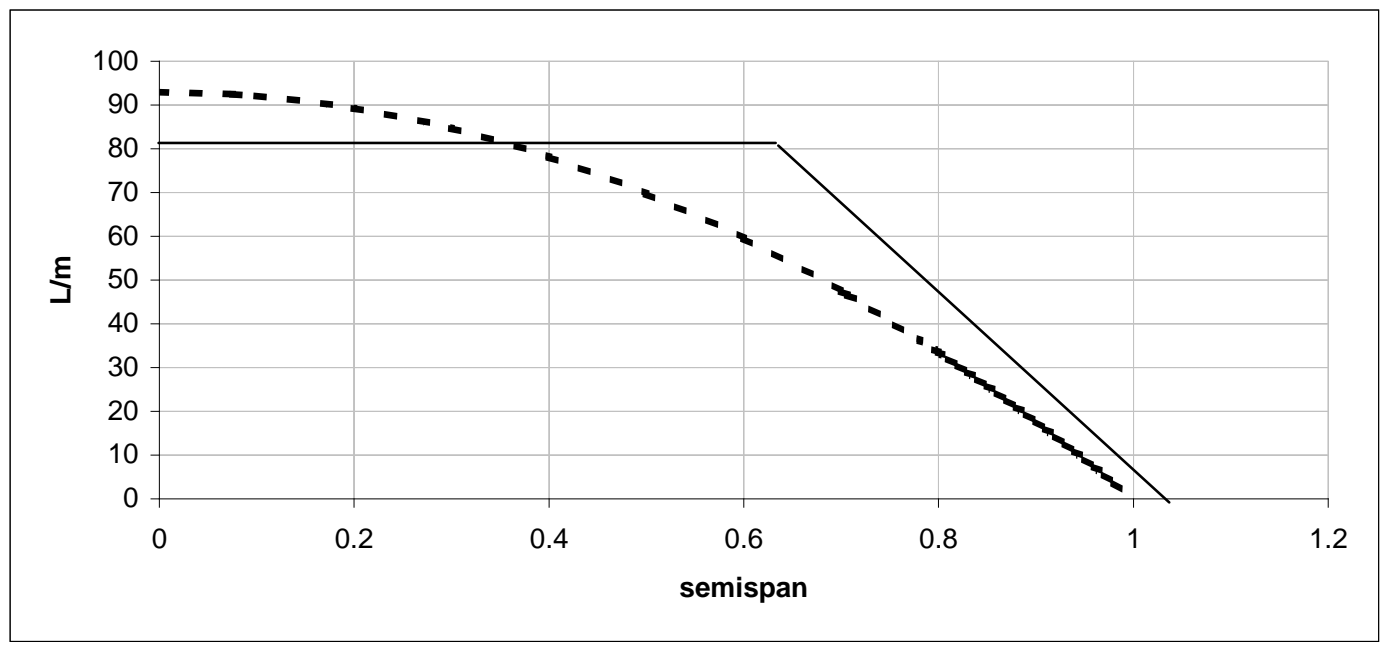

Fig. 44 Elliptical distribution vs. constant-linear lift distribution: This graph shows a comparison between the Elliptical distribution and the constant-linear distribution of the lift along the semi span. The constant linear distribution, constant from the root to the 2/3 of the semi span and then linear from the $2 / 3$ of the span to the tip of the wing, is introduced in order to simplify the calculations.

In the same fashion it is possible to calculate the lift $\mathrm{L}_{0}$ at the root:

$$
L=\int_{0}^{a} L(y) d y=L_{0}\left[\int_{0}^{2 a / 3} d y+3 \int_{2 a / 3}^{a}\left(1-\frac{y}{a}\right) d y\right]=\frac{5}{6} a L_{0} \Rightarrow L_{0}=\frac{6 L}{5 a}=87.6 \mathrm{~N} / \mathrm{m}
$$

It is also possible to make a first estimate of the total drag that will act on the wing. Using the Equation listed below

$$
\begin{aligned}
& D=\frac{1}{2} \rho V^{2} S C_{D} \\
& C_{D}=C_{D i}+C_{D o}
\end{aligned}
$$

$C_{D i}$ is the induced drag coefficient:

$$
C_{D i}=\frac{C_{L}^{2}}{\pi e A R}
$$

From the level flight condition

$$
\frac{1}{2} \rho V^{2} S C_{L}=L=W
$$


the $\mathrm{C}_{\mathrm{L}}$, at the critical condition of maximum speed, shown in Fig. 38, V = $25 \mathrm{~m} / \mathrm{s}$, at an altitude of $100-150 \mathrm{~m}$, is

$$
C_{L}=\frac{2 W}{\rho V^{2} S}=0.18
$$

Therefore the induced drag can be estimated, considering an $\mathrm{AR}=8$ and $\mathrm{e}=0.8$ (see Chapter III):

$$
C_{D i}=\frac{C_{L}^{2}}{\pi e A R}=0.0016
$$

Where $C_{D o}$ is the parasite drag coefficient and, as a first approximation, can be estimated using the flat-plate skin friction coefficient $C_{f}$ that depends upon the Reynolds number, Mach number and skin roughness. For a Reynolds number of 300 000, the $\mathrm{C}_{\mathrm{f}}$ for Laminar flow is:

$C_{f}=\frac{1.328}{\sqrt{\operatorname{Re}}}=0.0024$

and for turbulent flow is:

$$
C_{f}=\frac{0.455}{\left(\log _{10} \operatorname{Re}\right)^{2.58}\left(1+0.144 M^{2}\right)^{0.65}}=0.0057
$$

If the surface is relatively rough the friction coefficient will be higher.

Once the laminar and turbulent flat plate skin friction coefficients have been calculated, an average coefficient can be calculated, since at this point of the design it's difficult to make an estimate of the percentage laminar flow that can be attained: $\mathrm{C}_{\mathrm{f}}=0.0040$.

Considering for example $\frac{S_{\text {wet }}}{S_{\text {ref }}}=4$, which is the number estimated by [7] for a similar airplane configuration,

$$
C_{D 0}=C_{f} \frac{S_{\text {wet }}}{S_{\text {ref }}}=0.016
$$


The total drag coefficient, therefore, will be

$$
C_{D}=C_{D i}+C_{D o}=0.0176
$$

In order to consider the drag due to the small separation pressure drag and in order to take into account the approximations made using the theoretical equations, a safety coefficient of 1.5 is used. Finally $C_{D}=0.04$, and therefore the total drag is

$$
D=\frac{1}{2} \rho V^{2} S C_{D} \approx 7.7 \mathrm{~N}
$$

The drag calculated can be considered, with a good approximation, constant along the span as shown in Fig.41. The drag per unit length therefore is

$$
D_{0}=\frac{D}{a}=3.85 \mathrm{~N} / \mathrm{m}
$$

The loads calculated are represented in Fig. 45 where only the wing semi span is considered.
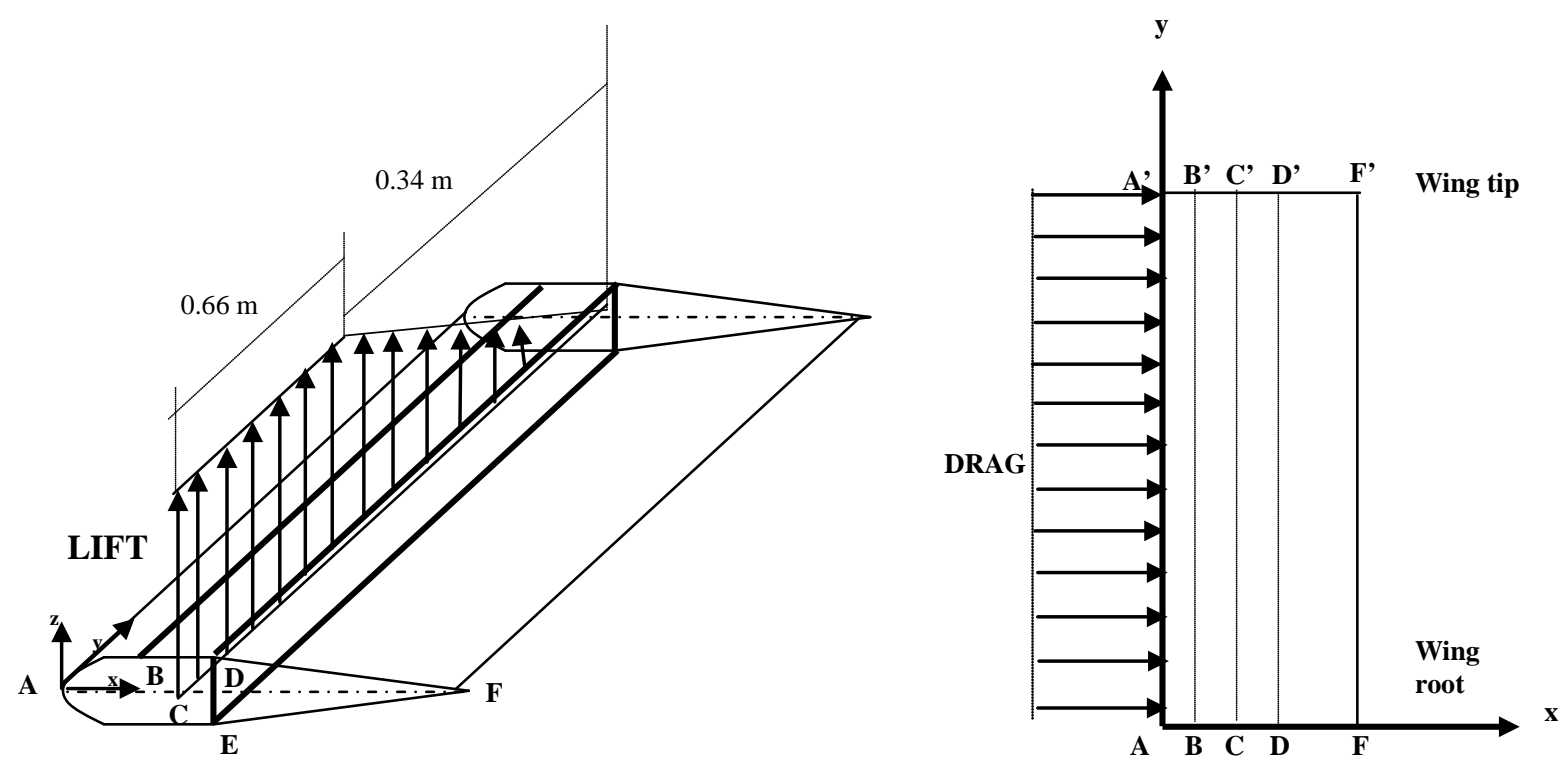

Fig. 45: Lift and drag distribution along the semi span. The lift is constant from the root to the 2/3 of the semi span and then linear from the 2/3 of the span to the tip of the wing. The drag is considered as a first approximation, constant along the span. 


\subsubsection{Structural analysis}

- The drag acting in the $\mathrm{x}$ direction, produces (at the $\mathrm{y}_{\mathrm{n}}$ section of the wing):

$\checkmark$ Shear Force $T_{x}$, parallel to the $\mathrm{x}$ axis applied through the shear centre

C

$$
T_{x}=\int_{y_{n}}^{a} D(y) d y=D_{0} a\left(1-\frac{y_{n}}{a}\right)
$$

The values of the shear force are plotted in Fig. 46

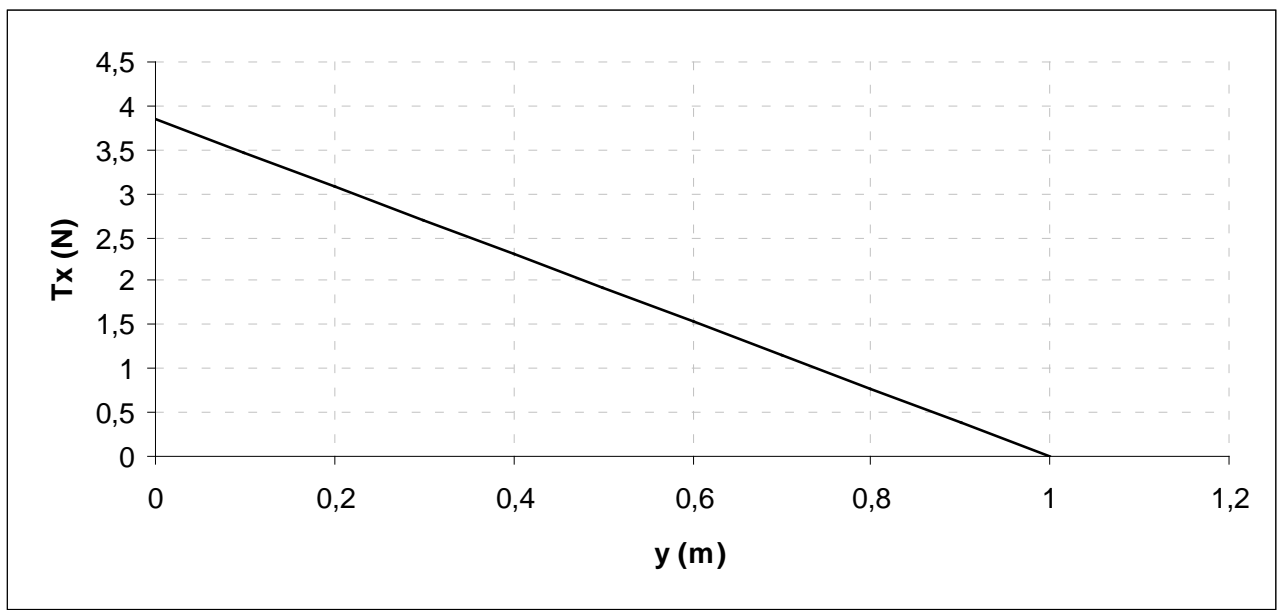

Fig.46 Shear force due to drag: One of the results of the drag acting in the $x$ direction is the shear force, parallel to the $x$ axis applied through the shear centre c. It has a linear behaviour with a maximum value of $3.8 \mathrm{~N}$.

$\checkmark$ Bending Moment $\mathrm{M}_{\mathrm{Z}}$ about an axis parallel to the $\mathrm{z}$ axis:

$$
M_{Z}=D_{0} \int_{y_{n}}^{a}\left(y-y_{n}\right) d y=\frac{D_{0} a^{2}}{2}\left[1-\frac{y_{n}}{a}\right]^{2}
$$

The values of the bending moment about the $\mathrm{z}$ axis are plotted in Fig. 47 


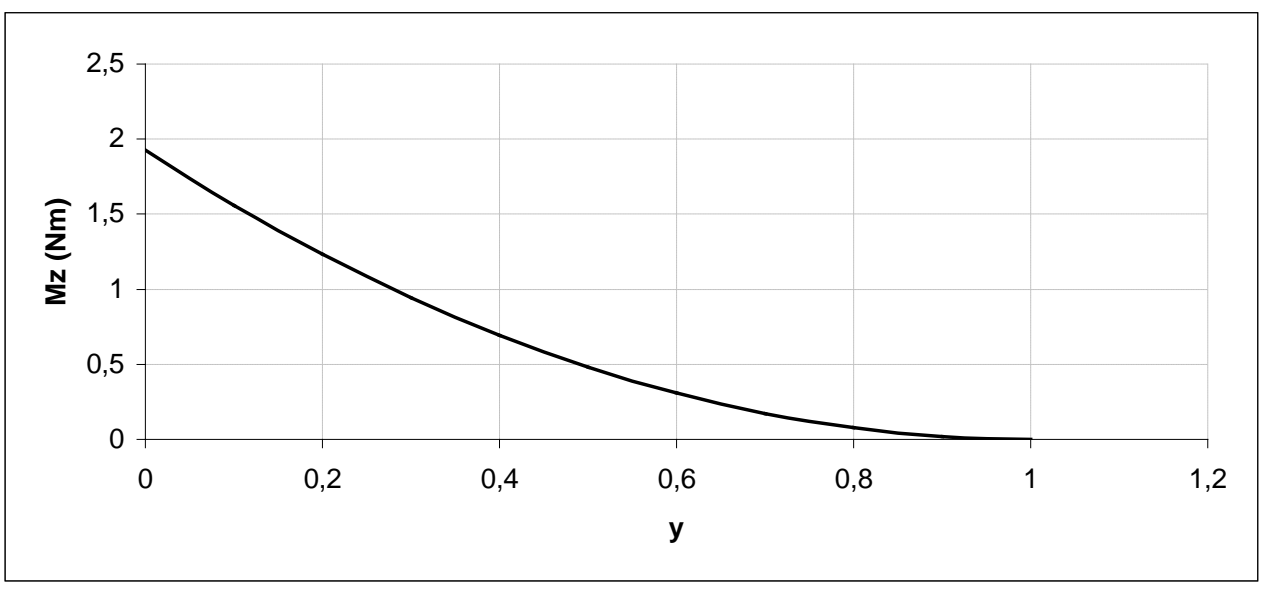

Fig.47 Bending moment due to drag: One of the results of the drag acting in the $x$ direction is the Bending Moment $M_{Z}$ about an axis parallel to the $z$ axis. It has parabolic behaviour with a maximum value of $1.8 \mathrm{~N}$.

- The lift acting in the $\mathrm{z}$ direction, produces (at the $\mathrm{y}_{\mathrm{n}}$ section of the wing):

$\checkmark$ A shear force $\mathrm{T}_{\mathrm{Z}}$ applied through the shear centre c:

$$
\begin{gathered}
T_{Z}=\int_{y_{n}}^{a} L(y) d y=L_{0}\left[\int_{y_{n}}^{2 a / 3} d y+3 \int_{2 a / 3}^{a}\left(1-\frac{y}{a}\right) d y\right]=L_{0} a\left[\frac{5}{6}-\frac{y_{n}}{a}\right] \rightarrow 0 \leq y_{n} \leq \frac{2 a}{3} \\
T_{Z}=\int_{y_{n}}^{a} L(y) d y=L_{0}\left[3 \int_{y_{n}}^{a}\left(1-\frac{y}{a}\right) d y\right]=\frac{3 L_{0} a}{2}\left[1-\frac{y_{n}}{a}\right]^{2} \rightarrow \frac{2 a}{3} \leq y_{n} \leq a
\end{gathered}
$$

The values of the shear force are reported in plotted in Fig. 48

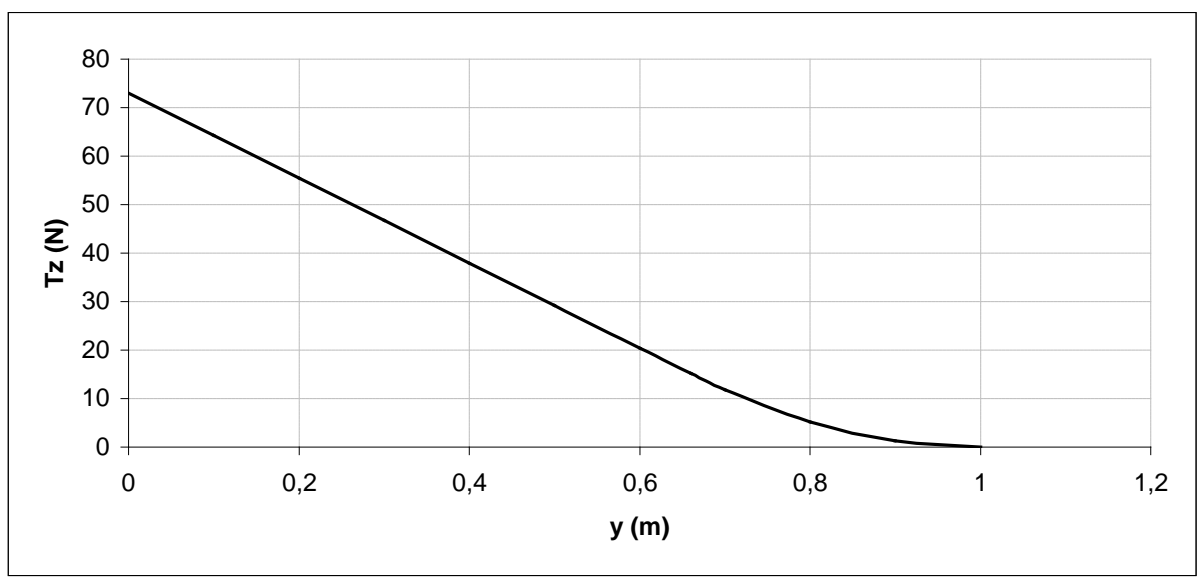

Fig.48 Shear force due to lift: One of the results of the lift acting in the z direction is a shear force in the z direction. It has a maximum value of $1.8 \mathrm{~N}$. 
Bending Moment $\mathrm{M}_{\mathrm{x}}$ about an axis parallel to the $\mathrm{x}$ axis:

$$
\begin{gathered}
M_{x}=\int_{y_{n}}^{a} L(y)\left(y-y_{n}\right) d y=L_{0} a^{2}\left[\frac{19}{54}-\frac{5}{6} \frac{y_{n}}{a}+\frac{y_{n}{ }^{2}}{2 a^{2}}\right] \rightarrow 0 \leq y_{n} \leq \frac{2 a}{3} \\
M_{x}=3 L_{0} \int_{y_{n}}^{a}\left(1-\frac{y}{a}\right)\left(y-y_{n}\right) d y=\frac{L_{0} a^{2}}{2}\left[1-\frac{y_{n}}{a}\right]^{3} \rightarrow \frac{2 a}{3} \leq y_{n} \leq a
\end{gathered}
$$

The values of the bending moment about the x-axis are plotted in Fig. 49

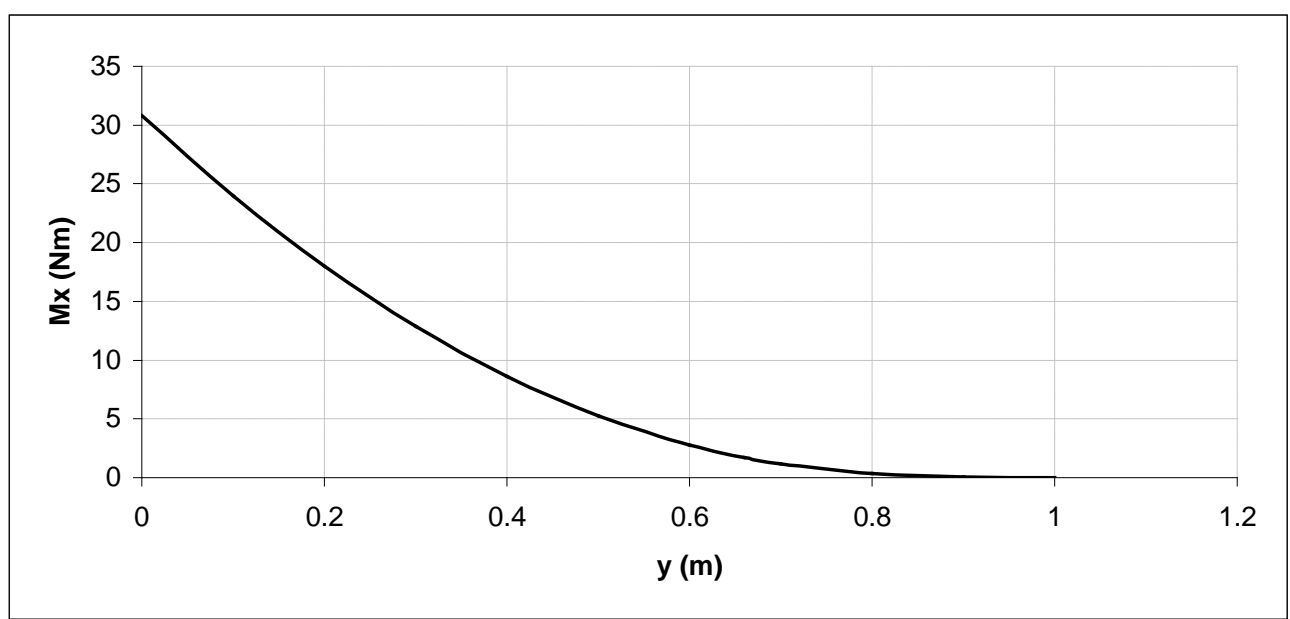

Fig.49 Bending moment due to lift: One of the results of the lift acting in the z direction is a Bending Moment $M_{x}$ about an axis parallel to the $x$ axis. It has a maximum value of $32 \mathrm{Nm}$.

As it appears clear from the Fig. 47, 48, 49 the root section of the wing undergoes the biggest stresses as expected.

The foam core and fibreglass were considered to not be bearing any of the stress, but merely transferring the stress to the carbon fibre spars, such that structurally, the wing could be modelled based on the properties of the spars.

\subsection{Nonplanar configuration vs. standard configurations}

Once the loads have been calculated and the general sizing of the wing has been completed, the possibility of a gain in weight due to the use of the nonplanar configuration under study is examined. 
Using the Finite Element software ADINA to predict stress and displacement, eight structural models were created, whose geometric, Fig. 50 and physical characteristics, Table 9, correspond to the eight wing configurations that have been tested in the Low Speed Wind Tunnel (LSWT) [18] of the University of Dayton.

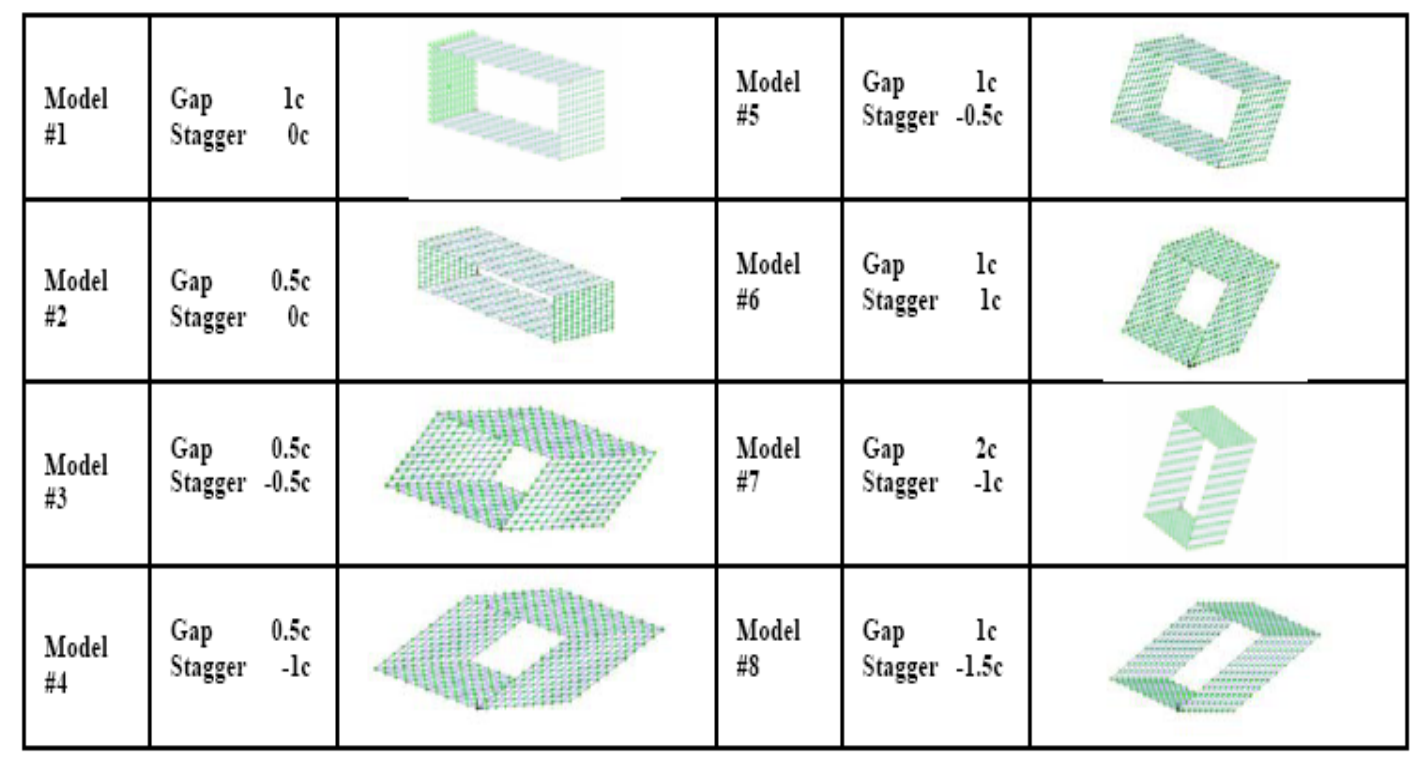

Fig.50: Finite Element Models and their geometric characteristics. Using the Finite Element software ADINA to predict stress and displacement, eight structural models were created, whose geometric and physical characteristics, Table 9, correspond to the eight wing configurations that have been tested in the Low Speed Wind Tunnel (LSWT) [18] of the University of Dayton.

\begin{tabular}{|l|l|}
\hline $\mathrm{C}$ & $0.10 \mathrm{~m}$ \\
\hline $\mathrm{b}$ & $0.60 \mathrm{~m}$ \\
\hline $\mathrm{t}$ & $1.27 \mathrm{~mm}$ \\
\hline $\mathrm{AR}=2 \mathrm{~b} / \mathrm{c}$ & 12 \\
\hline$\rho$ & $8000 \mathrm{Kg} / \mathrm{m}^{3}$ \\
\hline $\mathrm{E}$ & $200 \mathrm{Gpa}$ \\
\hline$v$ & 0.3 \\
\hline
\end{tabular}

Table 9 Physical characteristics of the models: this table represents the physical characteristics of the eight wing configurations that have been tested in the Low Speed Wind Tunnel (LSWT) [18] of the University of Dayton. The Aspect Ratio was calculated as 2b/c, the material used was stainless steel. 


\subsubsection{Methodology}

The initial construction of the base semis pan model with 0 stagger and 1 chord length gap begins with the definition of the model geometry. The points, the lines and the surfaces are defined, following the physical and geometric characteristics of the model as shown in Fig.51. For the surfaces the thickness is also specified according to Table 9.

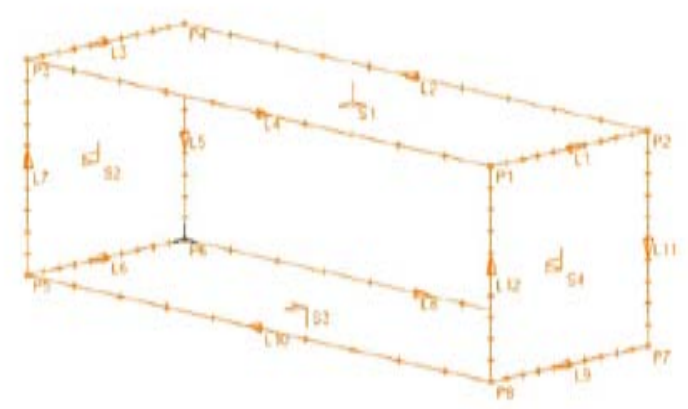

Fig.51 Adina: definition of the model: Using the finite element software ADINA, the first step is the definition of the model geometry. The points, the lines and the surfaces are defined, following the physical and geometric characteristics of the model. The model shown in this figure is model \#1 with 0 stagger and 1 gap.

Then the boundary conditions are applied. The semi span wing is assumed to be fixed to the fuselage and therefore in the finite element software ADINA, Surface 2 is specified as fixed.

In the Materials window, the properties of the selected material (Table 9) must be specified; these are the Young Modulus, Poisson's ratio and the density.

The loads must be also defined specifying the magnitude and the direction and the nodes of application. In this case the only load considered is the lift and an elliptical distribution is assumed. Furthermore a symmetrical lift distribution is 
assumed between the top and bottom wing and all the lift is considered to be applied at the quarter chord of each wing.

Finally the Finite Elements that can best approximate the behaviour of the structure under study are chosen. In this case, due to the small thickness of the surfaces in comparison with their other 2 dimensions, length and width, the Shell element seems to be the more appropriate. A uniform mesh density is then used with 10 by 10 divisions per each surface. Four nodes per element are considered in order to not exceed the privileges of the 900-node version of ADINA used. The resulting graphic window resembles Fig. 52.

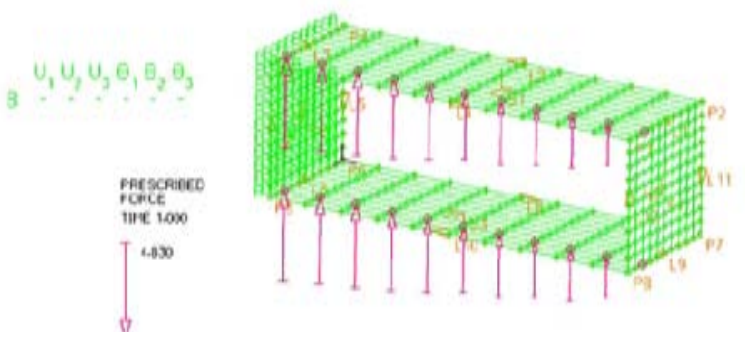

Fig. 52 Adina: mesh density creation: After approximating the behaviour of the structure with Shell elements, a uniform mesh density was created with four nodes per element. This figure shows the graphic window of ADINA for model 1 after the mesh.

After saving the model and running it in ADINA, the Post-Processing function must be opened in order to examine the solution. The displacements and stresses can easily be visualized in this way, in a table (Table 10) or through a band plot as shown in Fig. 53, 54. 


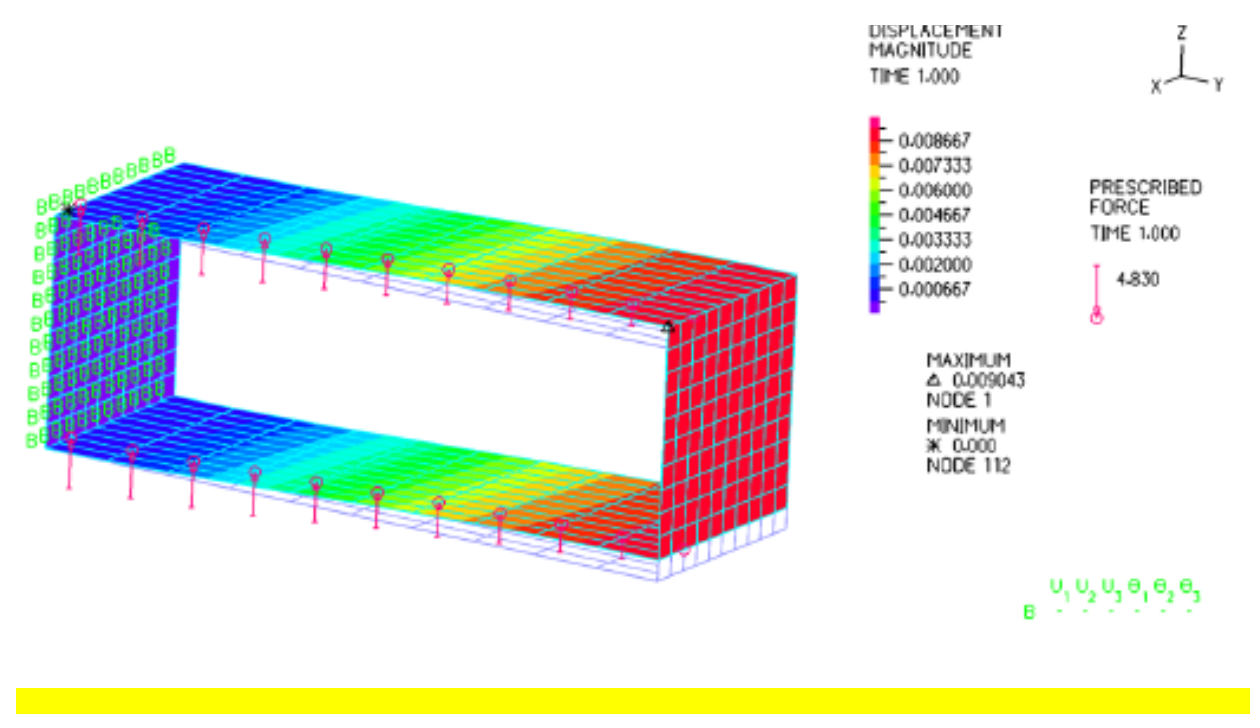

Fig. 53 Adina: examination of the solution: After saving the model and running it in ADINA, the PostProcessing function must be opened in order to examine the solution. The displacements can easily be visualized through a band plot. In this figure the band plot for model \#1 with 1 gap and 0 stagger is reported, which shows the displacement distribution. As could be predicted, the major displacement is on the wing tip.

Fig. 54 Adina: examination of the solution: After saving the model and running it in ADINA, the PostProcessing function must be opened in order to examine the solution. The stress can easily be visualized through a band plot. In this figure the band plot for model \#1 with 1 gap and 0 stagger is reported, which shows the stress distribution. As could be predicted, the major displacement is on the wing root. 
ADINA can also determine the first few natural frequencies and modes for the structure under study. In order to do this, it is necessary to remove the loads since the natural frequencies are independent of the loads applied and the number of the Frequencies/Mode shapes ADINA must determine must be specified.

After saving the new file and loading it into ADINA, a list of the natural frequencies can be displayed as shown in Fig. 55

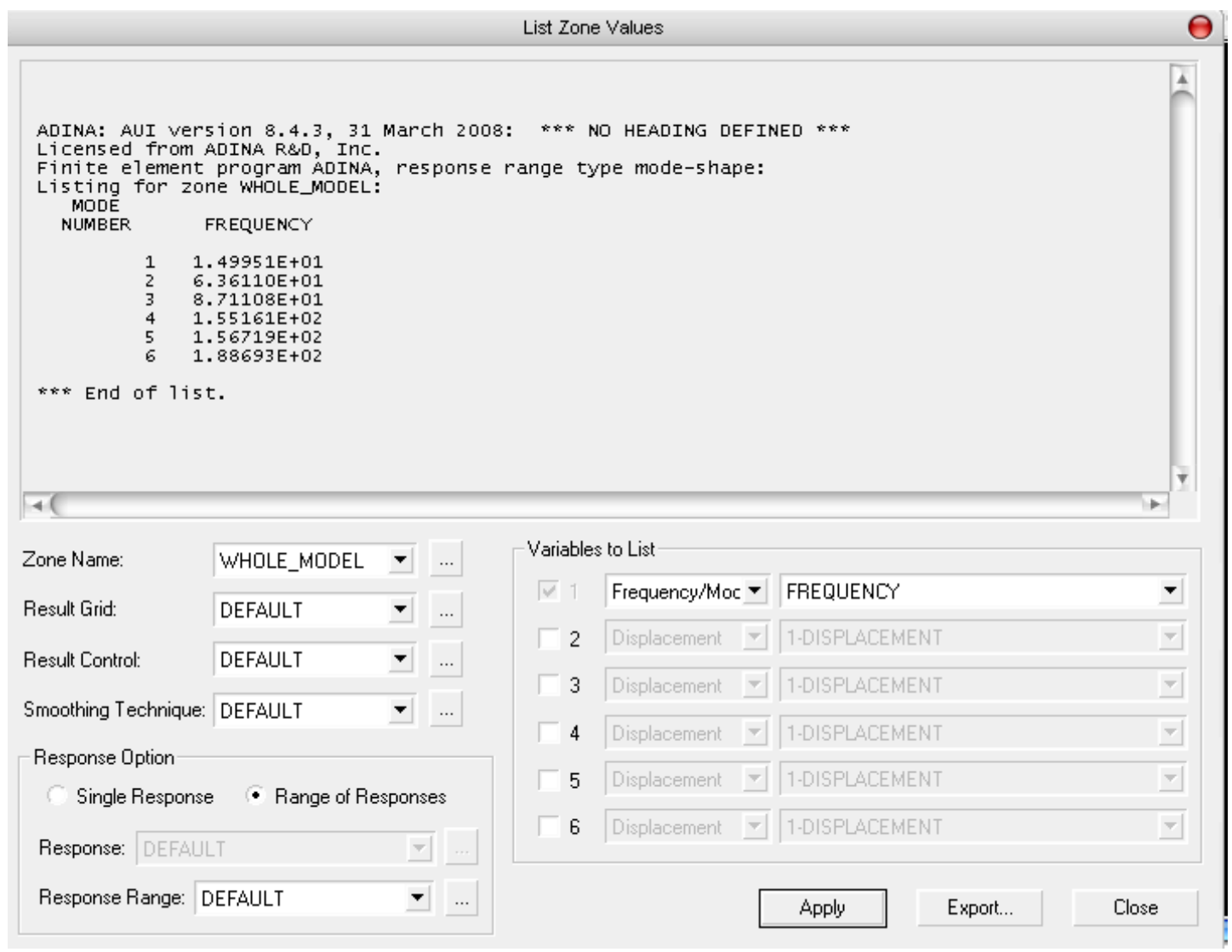

Fig. 55 Adina: natural frequencies: ADINA can also determine the first few natural frequencies and modes for the structure under study. A list of the natural frequencies can be displayed as shown in this figure. The first mode happens at $14.9 \mathrm{~Hz}$, the second at $63.6 \mathrm{~Hz}$ and the third at $87.11 \mathrm{~Hz}$.

It is also possible to plot the mode shapes and create a movie showing the model moving in its mode shapes. Fig.56 displays, the first mode shape of model \# 1 with 0 stagger and 1c gap as an example. 


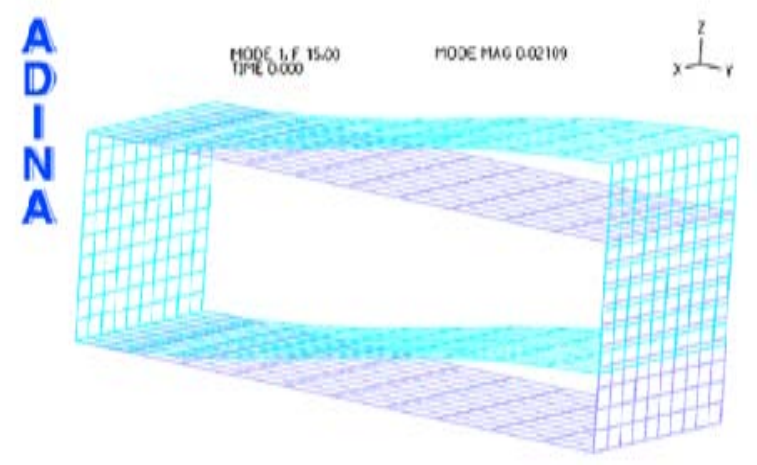

Fig. 56 Adina: mode shape: This figure displays the first mode shape of model \# 1 with 0 stagger and 1c gap as an example. In this case it represents the first bending mode shape. At higher frequency there is also the first torsion shape mode.

In the same fashion, changing only the geometric characteristics of the wing, it is possible to create the structural model of the other 7 nonplanar wing models tested in the UD Low Speed Wind Tunnel.

\subsubsection{Results}

Under the following assumptions:

$\checkmark$ Elliptical load distribution along the quarter chord of each wing

$\checkmark$ Symmetrical load distribution between the lower and the upper wing

$\checkmark$ Load applied at the quarter chord of each wing

$\checkmark$ Wing fixed to the wall on one side

the displacements and the maximum effective stresses were calculated and reported in Table 10. 


\begin{tabular}{|l|l|l|c|}
\hline Gap & Stagger & $\begin{array}{l}\text { Displacement } \\
(\mathbf{m})\end{array}$ & $\begin{array}{c}\text { Max Effective Stress } \\
\text { (MPa) }\end{array}$ \\
\hline 1 & 0 & 0.00904 & 54.8 \\
\hline 0.5 & 0 & 0.00868 & 55.0 \\
\hline 0.5 & -0.5 & 0.00897 & 55.6 \\
\hline 0.5 & -1 & 0.01012 & 57.2 \\
\hline 1 & -0.5 & 0.00926 & 55.2 \\
\hline 1 & -1 & 0.01002 & 56.4 \\
\hline 2 & -1 & 0.01032 & 56.7 \\
\hline 1 & -1.5 & 0.0115 & 58.7 \\
\hline
\end{tabular}

Table 10 displacement and maximum effective stress: Using the software ADINA, the displacement and maximum effective stresses were calculated for each configuration. It can be noticed that the configuration with 0.5 Gap and 0 Stagger has the smallest displacement and the model with 1 gap and 0 stagger has the smallest max effective stress.

For all of the models, the Maximum Effective Stress reported in Table 10 occurs at the root and the Maximum Displacement at the tip. From Table 10 it can be seen that the configuration with the smallest Maximum Effective Stress is model \#1 with 1C gap and 0 stagger; its stress distribution is reported in Fig. 54. On the other hand from Table 10 it can be seen that the configuration with the smallest displacement is model \#2 with $0.5 \mathrm{C}$ gap and 0 stagger, it's displacement distribution is reported in Fig. 57. 


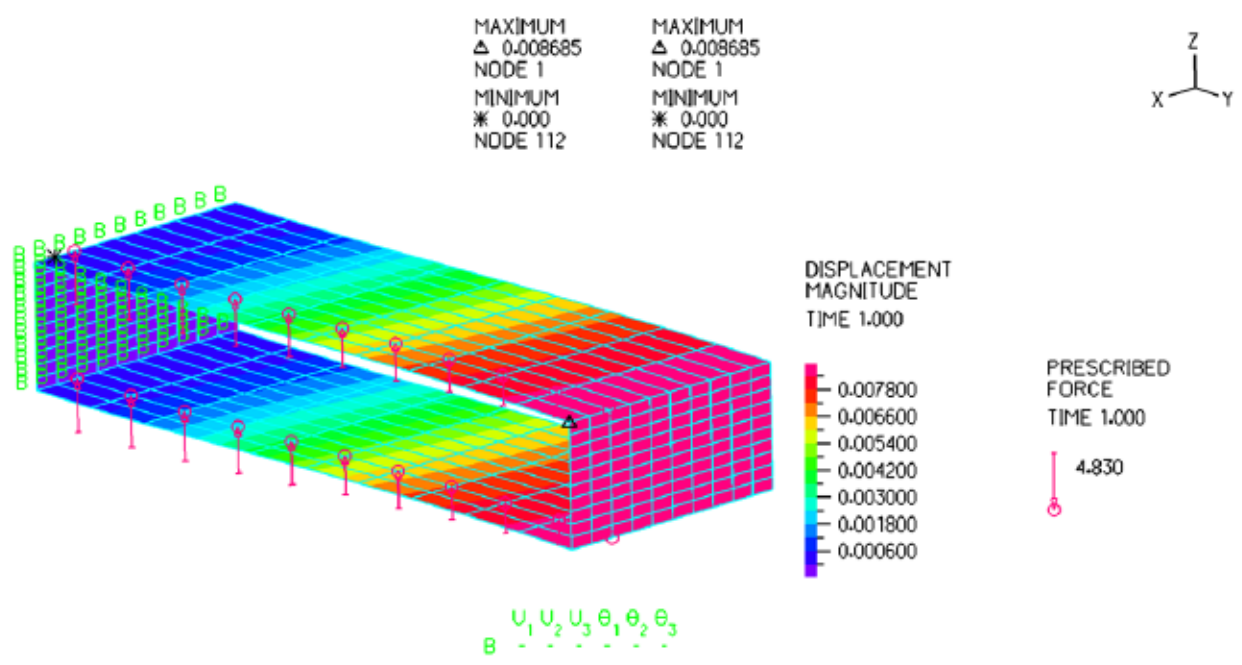

Fig. 57 Adina: configuration with the smallest displacement: from Table 10 it can be seen that the configuration with the smallest displacement is model \#2 with $0.5 \mathrm{C}$ gap and 0 stagger, it's displacement distribution is reported in this figure.

Furthermore it also appears clear from Table 10 that the model with the largest displacement and largest effective stress at the root is model \#8 with 1c gap and 1.5c stagger. Its stress and displacement distribution are reported in Fig. 58 and 59.

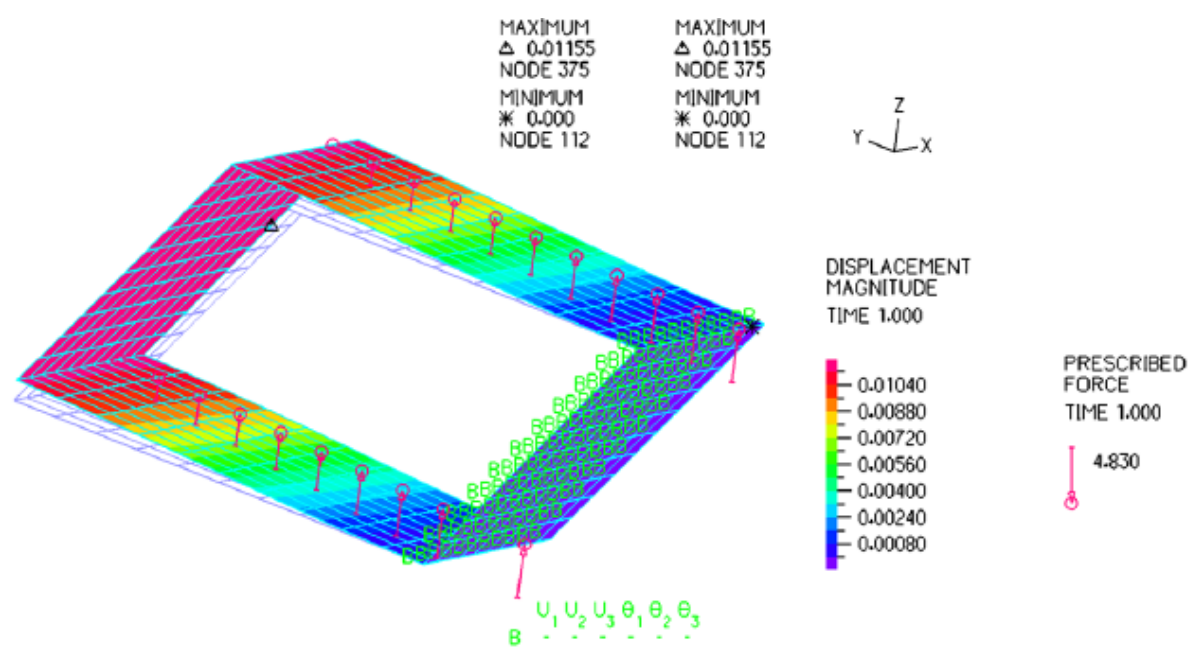

Fig. 58 Adina: configuration with largest displacement: from Table 10 that the model with the largest displacement and largest effective stress at the root is model \#8 with 1c gap and 1.5c stagger. Its displacement distribution is reported in this figure. 


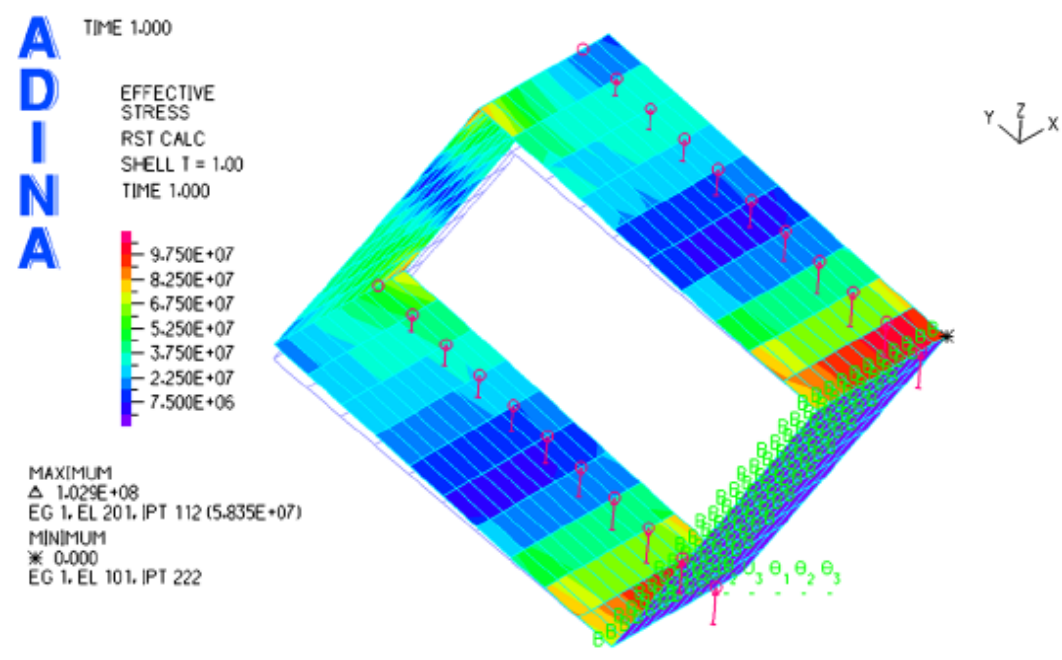

Fig. 59 Adina stress distribution of configuration with largest displacement and effective stress: from Table 10 that the model with the largest displacement and largest effective stress at the root is model \#8 with 1c gap and 1.5c stagger. Its stress distribution is reported in this figure.

It is also interesting to point out how a variation in the stagger and gap affects the structural behaviour of the multiple lifting surface configuration under investigation. This effect is clearly illustrated in Fig. 60 where the change in max effective stress with variation in the stagger while holding gap constant is reported.

From Fig. 60 it can be inferred that increasing the gap and therefore the size of the endplates at the tips of the biplane configuration has a beneficial effect on the structure, reducing the Maximum Effective Stress at the root. Furthermore increasing the stagger of the nonplanar configuration has a deteriorating effect on the structural performance of the wing under load. 


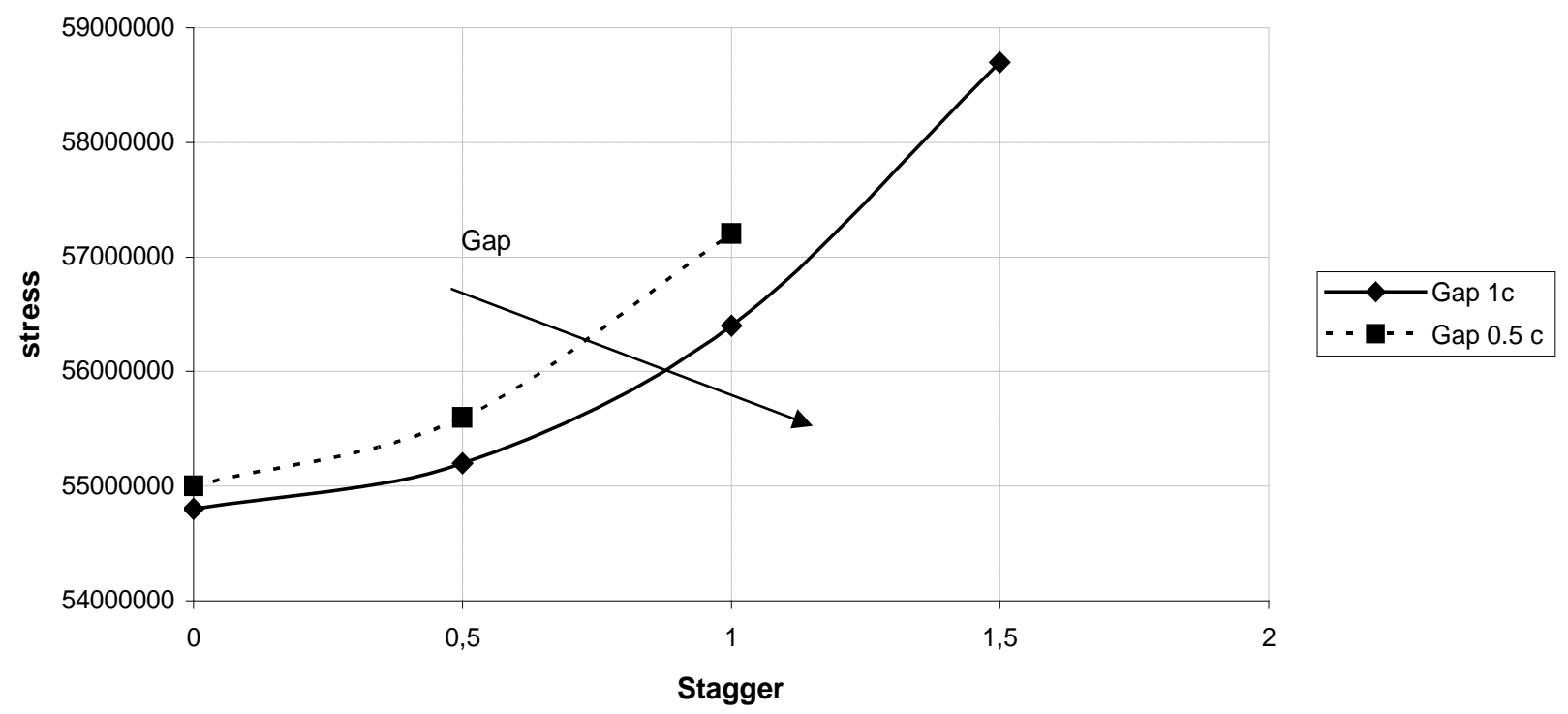

Fig. 60 Stress variation with gap and stagger: This figure shows a plot where the change in max effective stress with variation in the stagger while holding gap constant, is reported. Increasing the gap and therefore the size of the endplates at the tips of the biplane configuration has a beneficial effect on the structure, reducing the Maximum Effective Stress at the root. Furthermore increasing the stagger of the nonplanar configuration has a deteriorating effect on the structural performance of the wing under load.

\subsection{Comparison with a biplane}

In an effort to explore the structural benefits of the use of endplates, eight models of standard biplane not joined at the tips with flow guides were created using the same Finite Element software and the results, obtained under the same assumptions, were compared, in Table 11 and Fig. 61, to those reported in Table 10 and Fig. 60 


\begin{tabular}{|l|l|l|c|c|c|}
\hline Gap & Stagger & $\begin{array}{c}\text { Displacement } \\
(\mathbf{m})\end{array}$ & $\begin{array}{c}\text { Max Effective Stress } \\
\mathbf{( M P a )}\end{array}$ & $\begin{array}{c}\text { Displacement } \\
\text { Biplane (m) }\end{array}$ & $\begin{array}{c}\text { Max Effective Stress } \\
\text { Biplane (MPa) }\end{array}$ \\
\hline 1 & 0 & 0.00904 & 54.8 & 0.0247 & 80.6 \\
\hline 0.5 & 0 & 0.00868 & 55.0 & 0.0247 & 80.6 \\
\hline 0.5 & -0.5 & 0.00897 & 55.6 & 0.0247 & 80.6 \\
\hline 0.5 & -1 & 0.01012 & 57.2 & 0.0247 & 80.6 \\
\hline 1 & -0.5 & 0.00926 & 55.2 & 0.0247 & 80.6 \\
\hline 1 & -1 & 0.01002 & 56.4 & 0.0247 & 80.6 \\
\hline 2 & -1 & 0.01032 & 56.7 & 0.0247 & 80.6 \\
\hline 1 & -1.5 & 0.0115 & 58.7 & 0.0247 & 80.6 \\
\hline
\end{tabular}

Table 11 displacement and maximum effective stresses for a biplane: Using the software ADINA, the displacement and maximum effective stresses were calculated for eight models of standard biplane not joined at the tips with flow guides.. It can be noticed that the displacement and the Max Effective Stress for the biplane configuration remain the same for all the configurations.

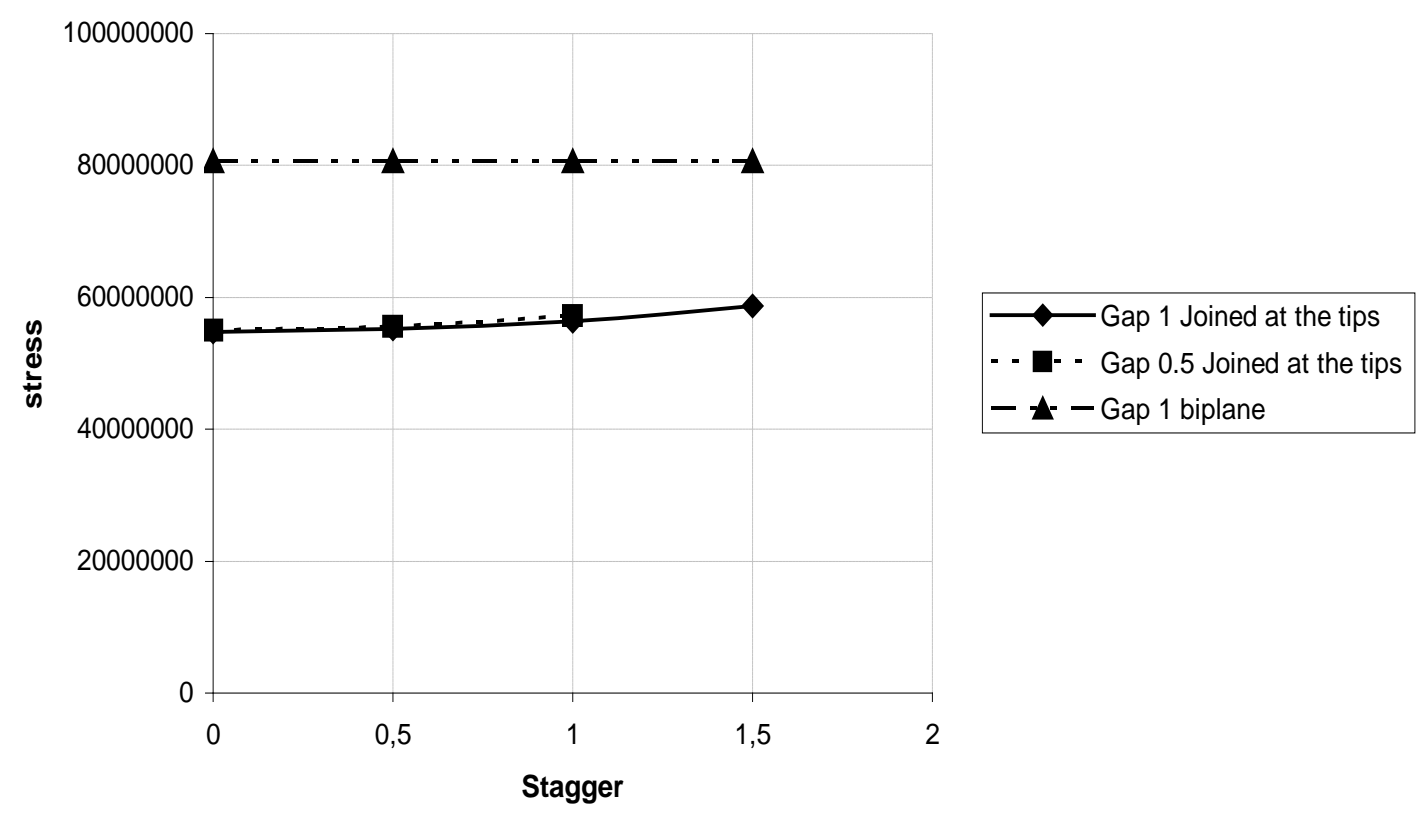

Fig. 61 Stress variation with stagger and gap: This figure shows a plot where the change in max effective stress with variation in the stagger while holding gap constant is reported for the standard biplane and for the configuration under study with a 0.5 and 1 gap. Increasing the gap the Displacement and the Maximum Effective stress of the biplane is constant. 
As expected, the Displacement and the Maximum Effective Stress of the biplane are constant with gap and stagger because the 2 wings are not joined and therefore behave independently from one another. As an example the stress distribution of the biplane with 0 stagger and 1c gap is reported in detail in Fig. 62.

Fig. 62 Adina: stress distribution of an equivalent biplane with 0 stagger and $1 \mathrm{c}$ gap: This figure represents in detail the Effective Stress distribution of an equivalent standard biplane with 0 stagger and $1 \mathrm{c}$ gap as obtained from the software ADINA.

Comparing the Maximum Effective Stress of model \#1 with 1c gap and 0 stagger to that of the biplane the reduction in stress is:

$$
\Delta \sigma \%=\frac{80,634,000-54,800,000}{80,634,000} \cdot 100=32.03 \%
$$

The price to pay for this reduction in stress is the increase in weight of the structure due to the addition of two endplates at the tips. This increase in weight can be easily quantified considering the physical characteristics of the configuration under investigation reported in Table 9, where the material used was the same structural material used for the wind tunnel models, stainless steel. Even though for the final design of the Small UAV another material will be chosen, the assumption of a 
material provides an important estimate of the gain or loss in weight this configuration can provide:

$W_{\text {biplane }}=\rho V_{\text {biplane }}=1.22 \mathrm{Kg}$

$W_{\text {endplates }}=\rho V_{\text {endplates }}=0.2 \mathrm{Kg}$

$W_{\text {Biplane_with_endplates }}=\rho V_{\text {biplane_with_endplates }}=1.41 \mathrm{Kg}$

$\frac{W_{\text {Biplane_with_endplates }}-W_{\text {Biplane }}}{W_{\text {Biplane_with_endplates }}} \cdot 100=13.5 \%$

Therefore in order to have a potential reduction in Maximum Effective Stress of $32.03 \%$ with a biplane configuration joined at the tips with endplates, there is an increase in weight of the $13.5 \%$. This can result in a dramatic advantage if the structural material of the wing is fixed by some constraints like the economic constraint.

\subsection{Comparison with an equivalent monoplane}

Two different comparisons with an equivalent monoplane are reported in this section using different perspectives in order to understand the potential advantages of using the nonplanar concept under study.

Using the same Finite Element software ADINA, a model of an equivalent monoplane was created under the assumptions of:

$\checkmark$ The same reference planform surface area (Lift capability) between the monoplane and the biplane joined at the tips with endplates

$\checkmark$ The same aspect ratio, therefore the same chord but different span: $b_{\text {monoplane }}=2 b_{\text {biplane_with_endplates }}$

The same total load

The same structural material 
$\checkmark$ Constant thickness along the span and the chord

$\checkmark$ Elliptical lift load distribution

In order to have the same Max Effective Stress at the root of model \#1 with 1C gap and 0 stagger, the thickness to chord ratio of the equivalent monoplane, using ADINA iteratively, is determined to be $40 \%$ larger than the biplane joined at the tips. The stress distribution for the equivalent monoplane is shown in Fig.63 in detail using the same layout used by ADINA.

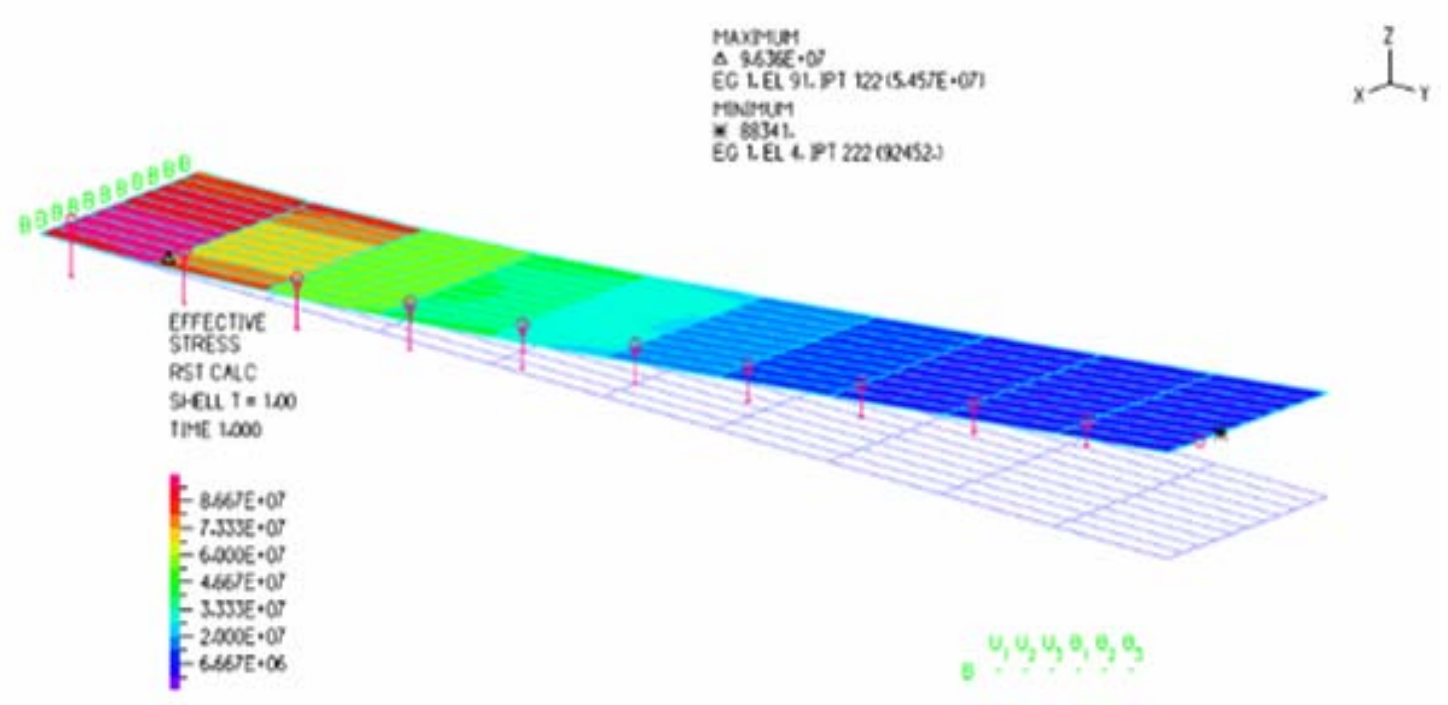

Fig. 63 Adina: Effective stress distribution of an equivalent monoplane: This figure represents in detail the Effective Stress distribution of an equivalent monoplane as obtained from the software ADINA when the wing undergoes to an elliptical lift distribution as calculated in the previous sections. The highest stress is located at the wing root.

The increase in thickness to chord ratio can be considered an estimate of the increase in weight of the structure and it can be easily quantified considering the physical characteristics of the configuration under investigation reported in Table 9: 


$$
\frac{W_{\text {monoplane_40\%thickness }}-W_{\text {Biplane_with_endplates }}}{W_{\text {monoplane_40\%thickness }}} \cdot 100=30.7 \%
$$

Therefore the consequent potential reduction in weight using the biplane configuration joined at the tips with a $1 \mathrm{C}$ gap and 0 stagger is around $30 \%$.

The stall constraint is used to consider the subsequent impact on the vehicle performance constraint:

$\frac{W}{S}=\frac{\rho_{S L}}{2} C_{L M A X} V_{\text {stall }}^{2}$

This potential reduction would translate into a lighter aircraft with a reduced stall speed and a longer endurance, all main keys for the mission profile of a Small Reconnaissance Surveillance Target Acquisition UAV. In fact solving the stall constraint equation just reported above for $\mathrm{V}_{\text {stall, }}$, a reduction in weight of $30 \%$, under the further assumption of same $C_{L \max }$, yields a reduction in the $\mathrm{V}_{\text {stall }}$ of $19 \%$. Since the target $V_{\text {stall }}$ for the design of the Small UAV is (Chapter IV) $9 \mathrm{~m} / \mathrm{s}$, this reduction would lead to a stall velocity of $7.30 \mathrm{~m} / \mathrm{s}$.

Given the aforementioned electric propulsion, the following relationship exists:

$$
P=I V=T V=D V=\frac{1}{2} \rho V^{2} S\left(C_{D o}+C_{D i}\right) V
$$

but

$$
C_{D i}=\frac{C_{L}^{2}}{\pi e A R}
$$

Substituting

$$
P=I V=T V=D V=\frac{1}{2} \rho V^{2} S\left(C_{D o}+\frac{C_{L}^{2}}{\pi e A R}\right) V
$$

In cruise

$$
C_{L}=\frac{2 W}{\rho V^{2} S}
$$


Substituting

$P=I V=\frac{1}{2} \rho V^{3} S C_{D o}+\frac{1}{2} \frac{\rho V^{3} S}{\pi e A R} \frac{4 W^{2}}{\rho^{2} S^{2} V^{4}}=\frac{1}{2} \rho V^{3} S C_{D o}+\frac{2 W^{2}}{\pi e A R \rho V S}$

Which shows the relationship between the power and the weight. Using the same parameters in the constraint equations in Chapter IV, the power required in cruise is: $\mathrm{P}=72 \mathrm{~W}$

And considering an efficency of the motor and of the propeller of 0.8 the power consumed in cruise is

$\mathrm{P}=90 \mathrm{~W}$

Using the electric motor AXI 4120/14 chosen in Chapter IV, with an operating voltage of $18.5 \mathrm{~V}$, and using a Lithium Polymer battery, with 5 cells in serial and 4 in parallel with a capacity of $8000 \mathrm{mAh}$, the endurance associated with this power in cruise is $I=\frac{P}{V}=4.86 \quad A=\frac{C(m A h)}{h} \rightarrow h=\frac{C(m A h)}{I}=1.64 h=98.6 \mathrm{~m}$

Considering all other parameters constant along with the potential reduction in weight of $30 \%$ from the biplane joined at the tips with endplates, the required power would be,

$\mathrm{P}=84 \mathrm{~W}$

And therefore

$I=\frac{P}{V}=4.54 \quad A=\frac{C(m A h)}{h} \rightarrow h=\frac{C(m A h)}{I}=1.76 h=105.7 \mathrm{~m}$

The endurance associated with the previous $90 \mathrm{~W}$ power requirements was $98.6 \mathrm{~m}$. In other words a reduction in weight of $30 \%$ would produce an increase in endurance equal to $6.6 \%$. 
Using the same Finite Element software ADINA, a model of a second equivalent monoplane was created under the assumptions of:

$\checkmark$ The same reference surface area (Lift capability) between the monoplane and the biplane joined at the tips with endplates

$\checkmark$ The same span: $b_{\text {monoplane }}=b_{\text {biplane_with_endplates }}$ and therefore different AR

$\checkmark$ The same total load

$\checkmark$ The same structural material

$\checkmark$ Constant thickness along the span and the chord

$\checkmark$ Elliptical lift load distribution

In order to have the same Maximum Effective Stress at the root of model \#1 with a $1 \mathrm{C}$ gap and 0 stagger, the thickness to chord ratio of the equivalent monoplane, determined using ADINA iteratively, is found to be $17 \%$ larger than that of the biplane joined at the tips. The stress distribution for the equivalent monoplane is shown in Fig.64 in detail using the same layout used by ADINA.

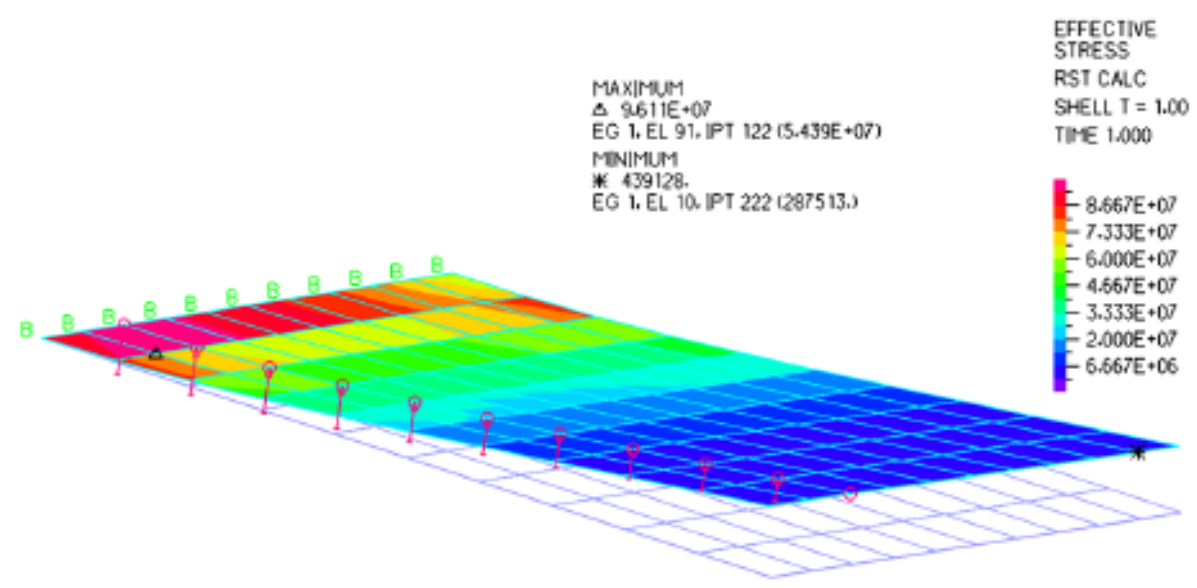

Fig. 64 Adina: Stress distribution of an equivalent monoplane with same span of the nonplanar configuration: This figure represents in detail the Effective Stress distribution of an equivalent monoplane with same span of the nonplanar configuration under consideration as obtained from the software ADINA when the wing undergoes to an elliptical lift distribution as calculated in the previous sections. The highest stress is located at the wing root. 
This increase in thickness to chord ratio translates into a reduction in weight if using the nonplanar configuration with wing joined at the tips given by:

$$
\frac{W_{\text {monoplane_17\%thickness }}-W_{\text {Biplane_with_endplates }}}{W_{\text {monoplane_17\%thickness }}} \cdot 100=3.5 \%
$$

This reduction can be used again to reduce the stall speed or to increase the endurance or, alternatively, to carry a heavier payload. This could be a heavier camera with better resolution or a camera with the same resolution but less expensive.

The biplane joined at the tips with endplates with 1c gap and 0 stagger, that gave the smallest Max Effective Stress from Table 10, will be compared in Chapter VI from an aerodynamic point of view to the equivalent monoplane just considered. The aim is always to exploit different points of view regarding the potential advantages of using a nonplanar concept and make the final configuration choice to follow in the next steps of the design process. 


\section{Chapter VI}

\section{Aerodynamic Analysis}

\subsection{Aerodynamics of a nonplanar wing configuration}

This section tries to explain the aerodynamic behaviour of the nonplanar configuration under examination. To this aim various analysis methods and comparisons were implemented. It can be possible that under some conditions, taking into account also the structural/weight considerations made in Chapter $\mathrm{V}$, the choice of a nonplanar configuration leads to some aerodynamic advantages in comparison to a standard monoplane.

In order to draw any aerodynamic conclusions, first the main results obtained using AVL software and in the Low Speed Wind Tunnel at the University of Dayton, [17], [18] and [31] are reported.

\subsubsection{Results obtained using AVL software and Wind Tunnel Testing}

In order to better understand the flowfield around the nonplanar configuration under study, a combination of vortex lattice method, integrated force measurement and stream wise PIV were used.

* A parametric study was performed using a Vortex Lattice code (AVL) on six parameters of the nonplanar configuration: stagger, gap, dihedral, decalage, sweep and overhang. The results obtained with AVL showed that the gap and stagger 
have the most dramatic effects (Fig. 65 and 66) out of the six parameters when aspect ratio and total wing area are kept constant. The effect of the other parameters was observed to be either negative or negligible.

The stagger, from the results of AVL, has a big impact on the lift coefficient. Fig. 65 shows the variation in lift coefficient with varying stagger at a 5-degree angle of attack. The $\mathrm{x}$-axis in the figure represents varying stagger from 0 to 2 chord lengths. The y-axis shows lift coefficient. The bottom solid line represents the smallest gap and it shows the largest deviation. The highest line is the largest gap and it shows the smallest deviation across the range of staggers. It is also noted that large variations continue up to $1 \mathrm{C}$, and very small changes occur beyond $1 \mathrm{C}$.

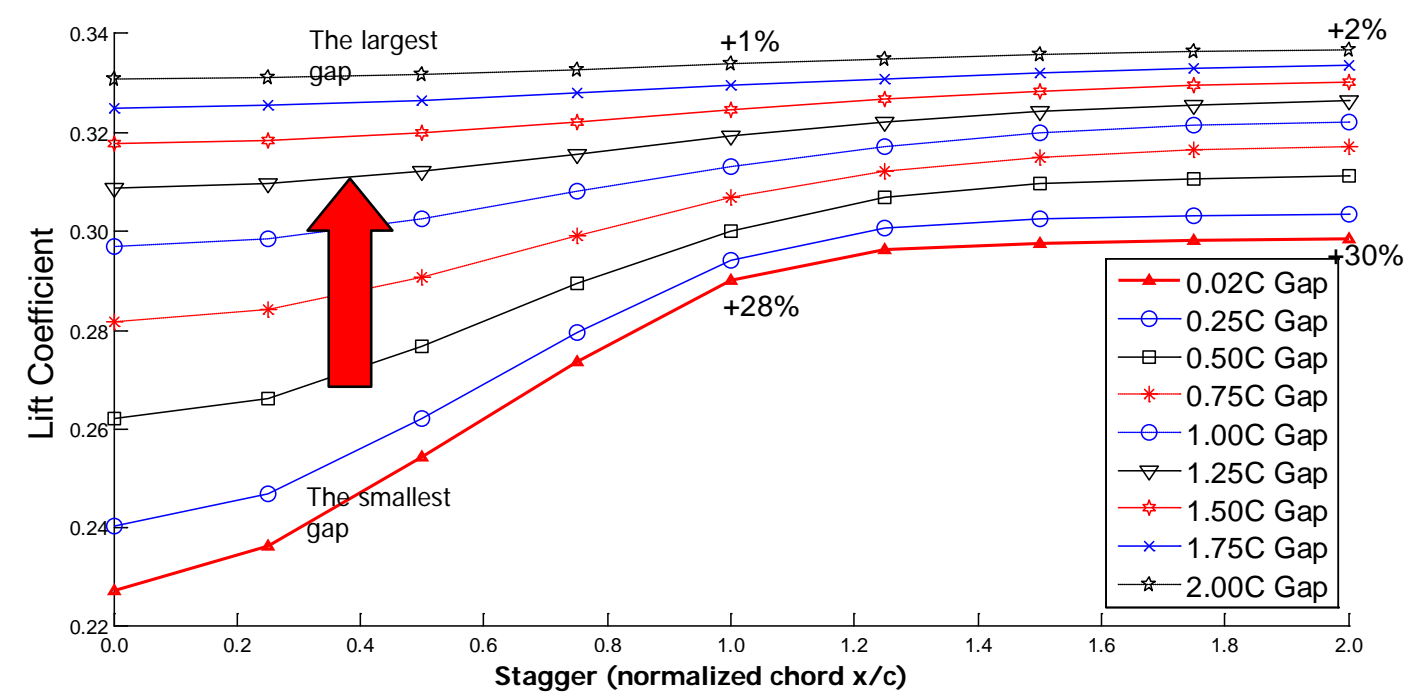

Fig. 65 Stagger vs. lift: Reported from [\#]: The stagger has a big impact on the lift coefficient. The graph shows the variation in lift coefficient with varying stagger at a 5-degree angle of attack. The $x$ axis in the figure represents varying stagger from 0 to 2-chord length. The y-axis shows lift coefficient. The bottom solid line represents the smallest gap and it shows the largest deviation. The highest line is the largest gap and it shows the smallest deviation across the range of staggers. 
The difference in lift coefficient between positive and negative stagger configurations is just $0.01 \%$ through AVL analysis. This difference can be attributed to computational precision and the difference is thus considered to be zero.

From the results of AVL the gap has a big impact on the lift coefficient too (Fig. 66). Increasing the gap between the two lifting surfaces of a biplane will result in an increase in the total lift coefficient. A greater rate of increase in lift coefficient as a function of increasing gap is observed until the gap reaches approximately 1 chord length distance. Above one chord length gap, the rate of change of lift coefficient decreases with increasing gap. Further increases in the gap result in minimal interaction between the wings and leads to the lifting surfaces acting individually.

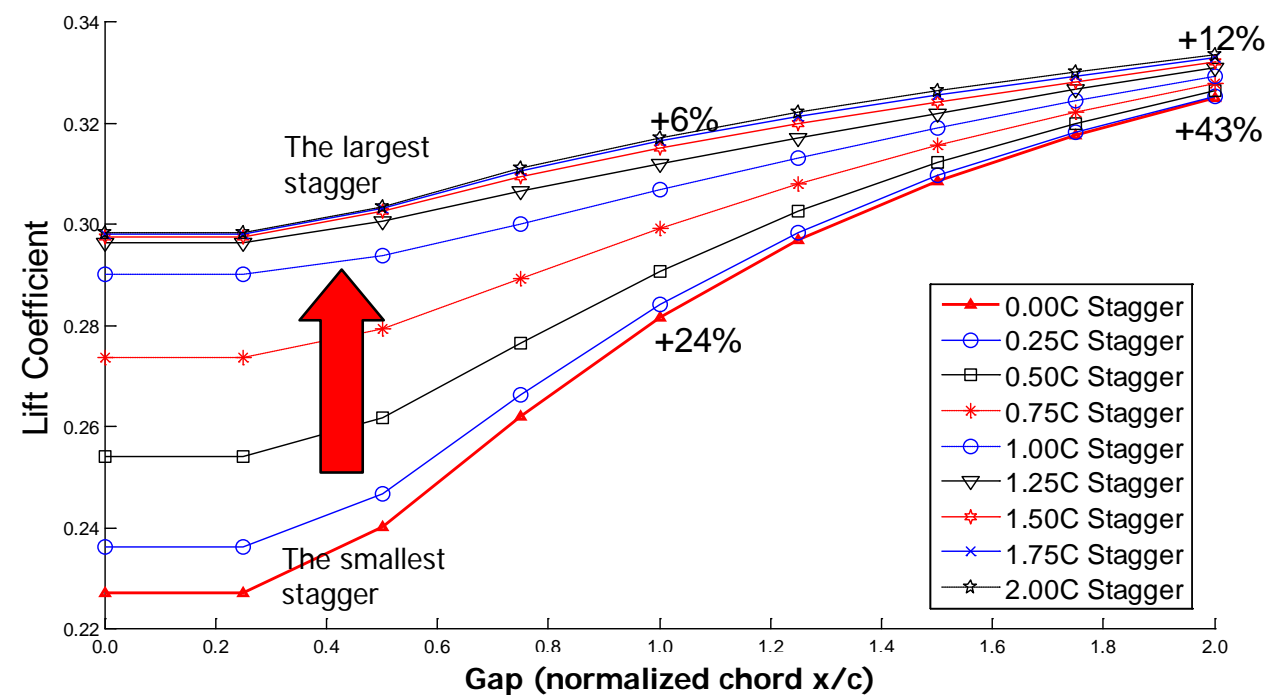

Fig. 66 Gap vs lift reproduced from [\#]:The gap, from the results of AVL has a big impact on the lift coefficient. Increasing the gap between the two lifting surfaces of a biplane will result in an increase in the total lift coefficient. A greater rate of increase in lift coefficient as a function of increasing gap is observed until the gap reaches approximately 1 chord length distance.

From the results obtained from AVL, the parameters were reduced to gap and stagger for wind tunnel testing.

* The experimental results were obtained from force balance measurement. 
They show some differences from the results obtained from AVL, due to the underlying assumptions of this code: incompressible, inviscid, irrotational and steady flow.

The force balance measurements show that as stagger increases in the positive direction the lift coefficient also increases. A large variation in lift behaviour was found between the positive and negative stagger configurations. A positive stagger can produce a lift coefficient $47 \%$ higher than a negative stagger configuration (for a 0.5 C gap, which was the largest variation).

Fig. 67 compares lift coefficient variation with angle of attack measured during wind tunnel testing with the results from the Vortex Lattice code (AVL) for the configurations with 0C stagger and 1C gap, and 0.5C gap. From Fig. 67, for the configuration with 0 stagger and $1 \mathrm{C}$ gap, measured $\mathrm{C}_{\mathrm{L}}$ from the force balance was higher than predicted by AVL across the range of angle of attack until stall. The configuration with 0C stagger and 0.5 gap, shows force balance measurements close to the AVL results with gradual stall characteristics after $10^{\circ}$ angle of attack.

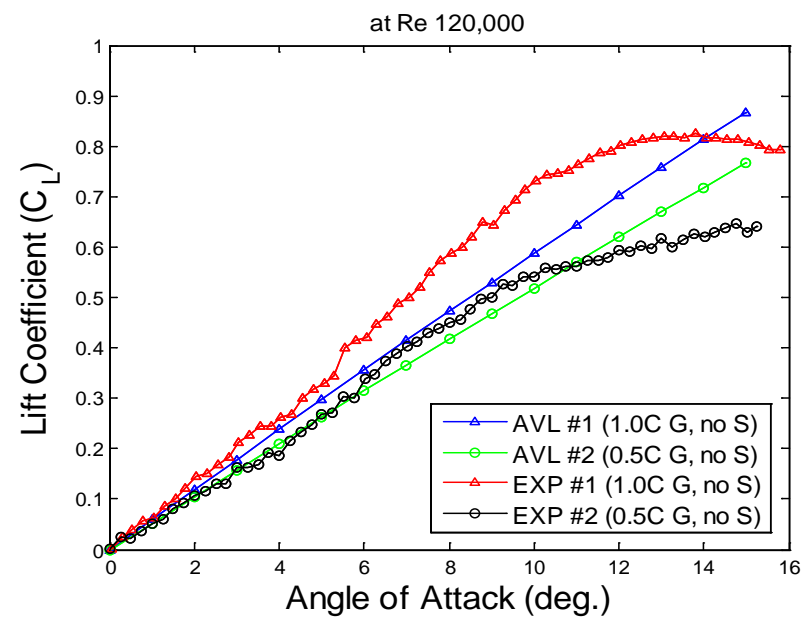

Fig.67 AVL lift vs angle of attack: AVL results and the experimental results show differences due to the assumptions of AVL. This graph compares lift coefficient variation with angle of attack measured during tunnel testing with the results from the Vortex Lattice code (AVL) for the configurations with 0C stagger and $1 C$ gap, and $0.5 C$ gap. 
From a design point of view, it is interesting to find the configuration with the highest aerodynamic performance that meets the requirements of the Small UAV under design. Due to the long endurance requirement, the parameter to evaluate for different stagger and gap, is the lift to drag ratio, which represents the aerodynamic efficiency of the airplane: the higher the $\mathrm{L} / \mathrm{D}$, the lower the glide angle, and the greater the distance that an airplane can travel across the ground for a given change in height. Actually, this is incorrect. If you look at the difference between the range and the endurance equations for propeller driven a/c, the range is a direct function of $\mathrm{L} / \mathrm{D}$ as you say, but the endurance/loiter is a function of $\mathrm{CL}^{\wedge}(1.5) / \mathrm{CD}$.

Therefore the figures below report the behaviour of the lift to drag ratio when varying stagger and gap.

Fig. 68 shows the lift to drag ratios versus the angle of attack. As stagger increases, aerodynamic characteristics improve dramatically beyond an angle of attack of $4^{\circ}$. In particular, this research focuses on the mission profile of a Small Reconnaissance UAV. Therefore, considering its proposed physical characteristics derived in chapter IV, with a weight at takeoff of $3 \mathrm{Kg}$ and a reference surface area of $0.5 \mathrm{~m}^{2}$, for a cruise speed of $18 \mathrm{~m} / \mathrm{s}$, a design $\mathrm{C}_{\mathrm{l}}$ of 0.25 can be calculated. The angle of attack that corresponds to this flight configuration, considering a flat plate, would be $2.56^{\circ}$. The $0 \mathrm{C}$ stagger configuration has the best performance in those range of angles of attack.

Fig. 69 shows how the gap affects the lift to drag ratios. In these plots, the highest $\mathrm{L} / \mathrm{D}$ ratio is obtained near an angle of attack of $4^{\circ}$. From the tunnel testing, it is obvious that increasing the gap on the biplane with endplates increases the lift force and reduces the lift-induced drag, improving the lift-to-drag ratio. On the other side, as seen in Table 10 in Chapter V, from a structural point of view it is not desirable to 
have a big gap between the 2 wings because the structure undergoes higher bending loads. Therefore a compromise was chosen, and the configuration with $1 \mathrm{C}$ gap and 0C stagger was chosen for an aerodynamic comparison to an equivalent monoplane.

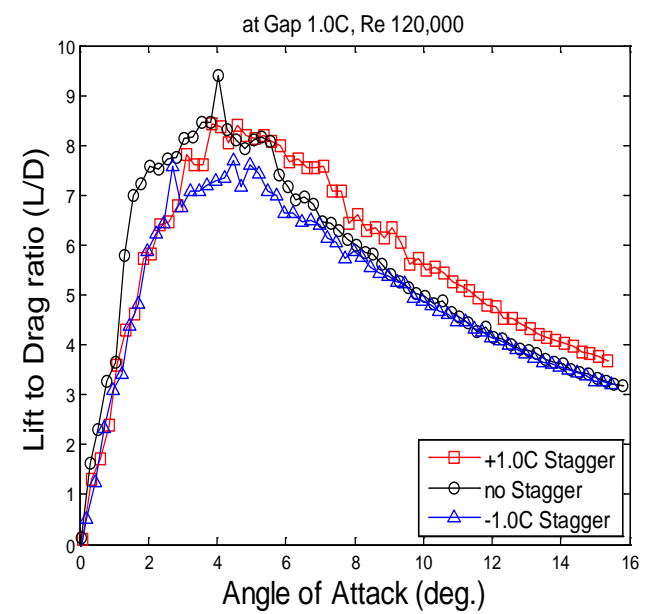

Fig.68 Experimental results $L / D$ vs. angle of attack for different stagger configurations: This figure shows the experimental results obtained in the Low Speed Wind Tunnel at the University of Dayton. In particular it shows the lift to drag ratios versus the angle of attack for different Stagger configurations. As stagger increases, aerodynamic characteristics improve dramatically beyond an angle of attack of $4^{\circ}$.

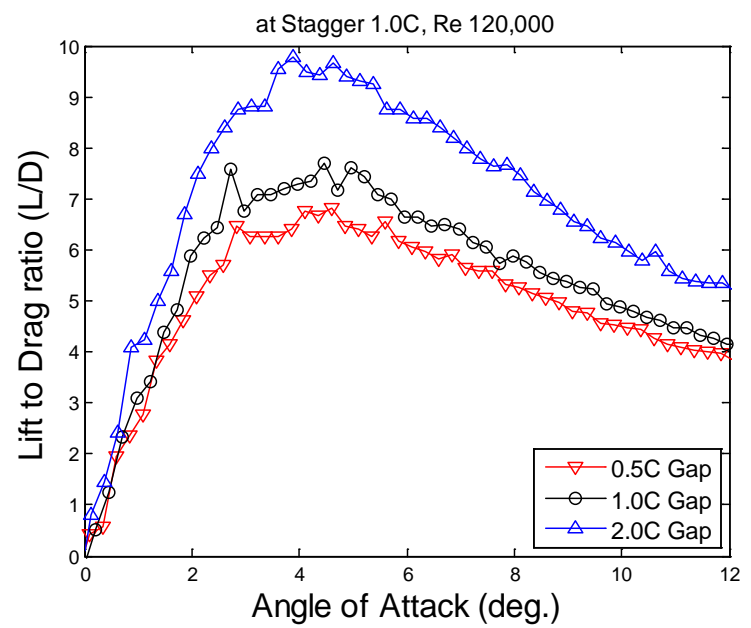

Fig. 69 Experimental results $L / D$ vs angle of attack for different gap configurations: This figure shows the experimental results obtained in the Low Speed Wind Tunnel at the University of Dayton. In particular it shows the lift to drag ratios versus the angle of attack for different gap configurations. Increasing the gap on the biplane with endplates increases the lift force and reduces the lift-induced drag, improving the lift-to-drag ratio. 
6.1.2 Aerodynamic comparison between an equivalent monoplane and the nonplanar configuration under study

Taking into account the structural/weight considerations made in Chapter V it is possible that under some conditions, the choice of a nonplanar configuration leads to some aerodynamic advantages in comparison to a standard monoplane. As anticipated in the previous section, the nonplanar configuration taken into consideration is the 0C stagger 1C gap configuration.

Using the vortex panel code AVL (Athena Vortex Lattice) under the incompressible, inviscid, irrotational and steady flow assumptions, an aerodynamic model (Fig. 70) of the equivalent monoplane considered in Chapter V, section 5.2 (Fig. 60). The geometric and physical characteristics are as follows:

\footnotetext{
$\checkmark$ Chord: $\mathrm{c}=0.2 \mathrm{~m}$

$\checkmark$ Span: $b=0.6 \mathrm{~m}$

$\checkmark$ Reference platform area: $0.12 \mathrm{~m}^{2}$

$\checkmark$ Flat plate profile

$\checkmark \quad \operatorname{Re}=120,000$
} 


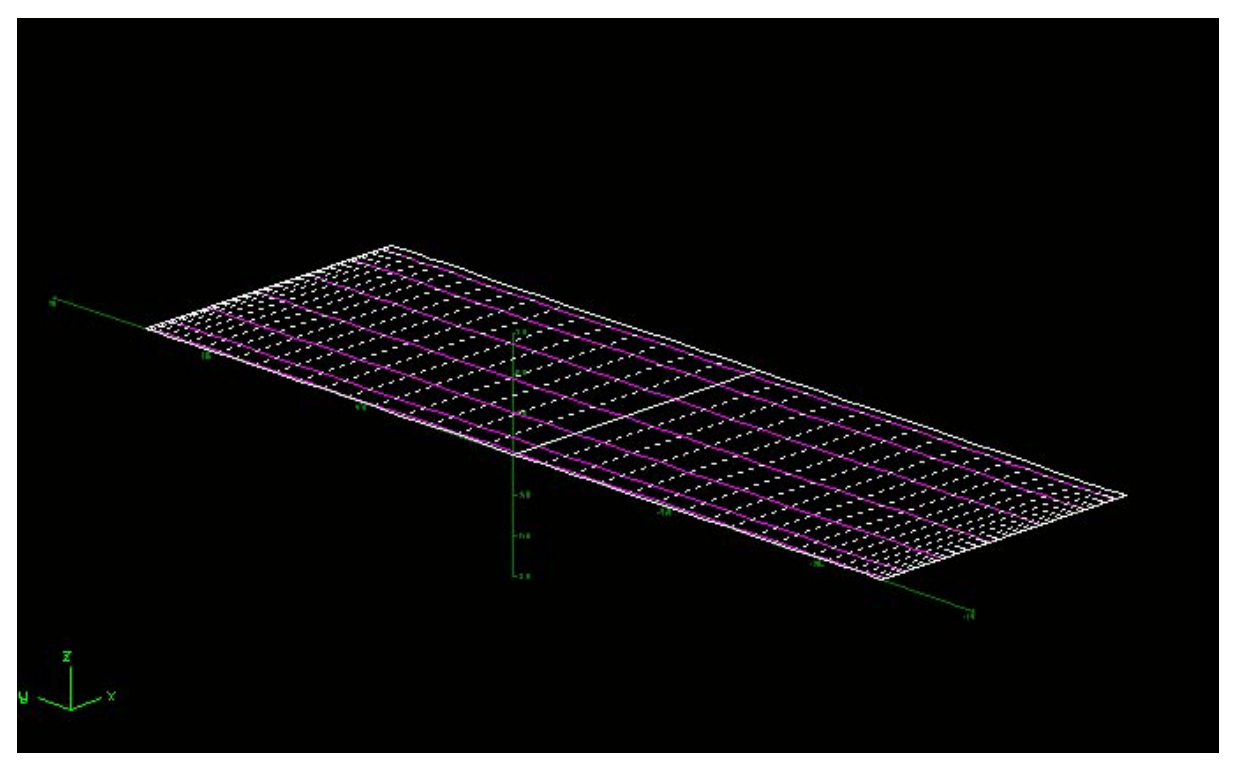

Fig. 70 AVL equivalent monoplane geometry: In order to make a final choice about which wing configuration should be used for the Small UAV under design, an equivalent monoplane was considered and modelled with the software AVL, and then compared to the nonplanar configuration under study. This figure shows the displayed image on the software AVL after all the geometrical inputs have been inserted in the code.

Figure 71 shows a comparison in the lift slope between the two configurations. The lift slopes of the biplane joined at the tips and the monoplane are 0.072 and 0.054 respectively with a consequent gain in lift slope for the biplane joined at the tips:

$$
\Delta \frac{d C_{L}}{d \alpha}=24.7 \%
$$

It's important to note that this considerable gain in lift slope is in part due to an unusual change in the lift slope of the biplane with endplates that can be observed around $5^{\circ}$. Further investigation is required to better understand this unusual behavior. Considering the linear portion of the lift curve until $5^{\circ}$, the lift slope equals 0.065 with a gain in lift, in this case, equal to:

$$
\Delta \frac{d C_{L}}{d \alpha}=16.5 \%
$$




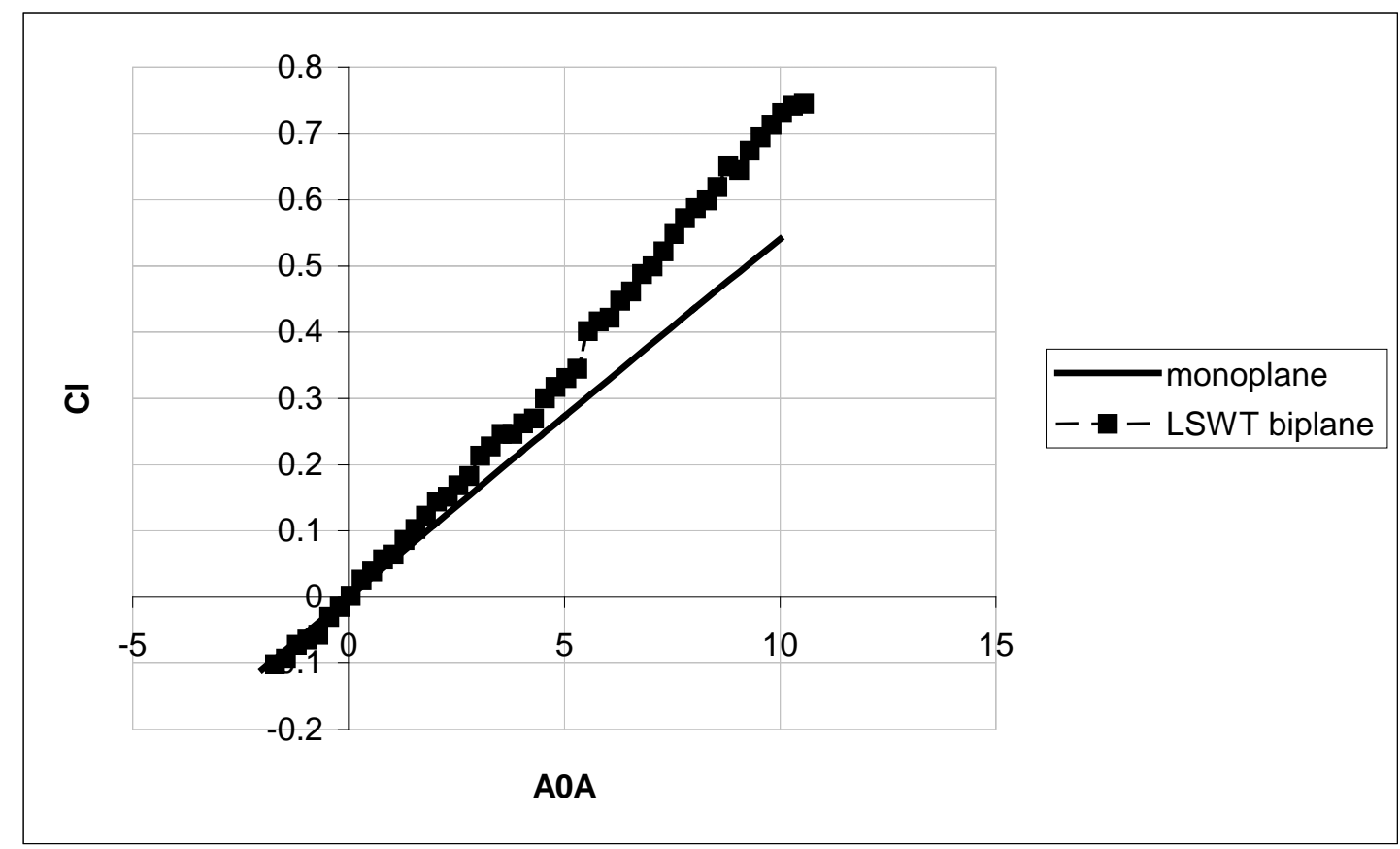

Fig. $71 \mathrm{Cl}$ vs. angle of attack: monoplane vs biplane: In order to make a final choice about which wing configuration should be used for the Small UAV under design, an equivalent monoplane was considered and modelled with the software AVL, and then compared to the nonplanar configuration under study. This figure shows a comparison in the lift slope between the two configurations.

Fig. 72 shows the variation of the Lift to Drag ratio with the Angle of Attack for the two configurations under consideration. For small values in angle of attack, from $0^{\circ}$ to $4^{\circ}$, the biplane configuration shows higher aerodynamic efficiency. These results will now be related back to the mission profile of a Small Reconnaissance UAV. Considering the proposed physical characteristics derived in [3] with a takeoff weight of $2.9 \mathrm{Kg}$ and a reference surface area of $0.5 \mathrm{~m}^{2}$, for a cruise speed of $18 \mathrm{~m} / \mathrm{s}$, a design $C_{L}$ of 0.28 is calculated. The angle of attack that corresponds to this flight configuration, considering a flat plate, would be $2.56^{\circ}$. The gain in Lift to Drag ratio for this particular angle of attack would be: 


$$
\Delta\left(\frac{L}{D}\right)=\left(\frac{L}{D}\right)_{\text {biplane }}-\left(\frac{L}{D}\right)_{\text {monoplane }}=\frac{7.71-6.38}{7.71}=17.25 \%
$$

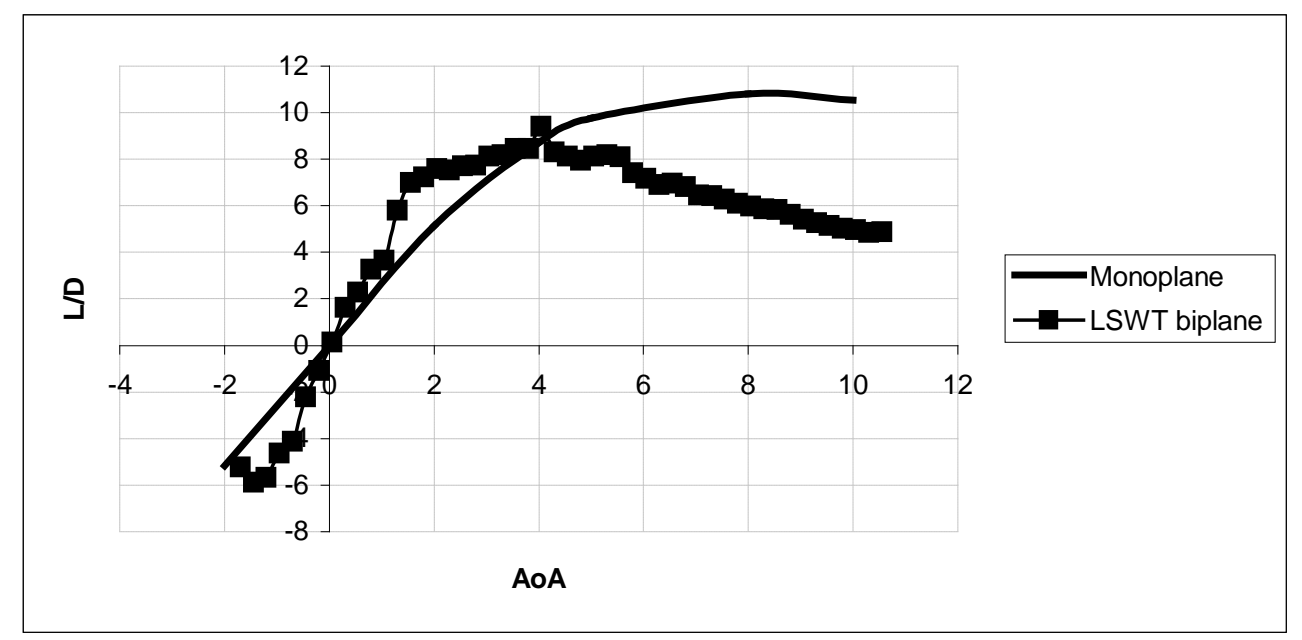

Fig. 72 L/D vs. angle of attack: monoplane vs. biplane: In order to make a final choice about which wing configuration should be used for the Small UAV under design, an equivalent monoplane was considered and modelled with the software AVL, and then compared to the nonplanar configuration under study. This figure shows a comparison in the $L / D$ ratio vs. angle of attack between the two configurations.

It must be taken into account that the results from AVL are valid under the irrotational, incompressible steady and inviscid flow assumptions. The only viscous coefficient considered from AVL is $\mathrm{Cd}_{0}$ whose value must be entered by the user. In this case a historically representative value of 0.02 was used. In the case for which a greater $\mathrm{Cd}_{0}$ is assumed, the overall drag of the monoplane would increase, its performance and the lift-to-drag ratio would decrease, and therefore the region (shown in Fig.72) in which the biplane configuration would perform better than the monoplane would increase. 


\subsection{Decision Analysis}

In order to choose a final configuration for the Small UAV, a decision analysis is performed. The configuration with the best score is used in the following steps of the design process. Five criteria are considered and for each one a "weight" from 1 to 5 is associated as reported in Table 12.

$\checkmark \quad$ The configurations analyzed are the configurations compared through this research, that is, the Biplane Joined at the tips, non planar configuration with 0C stagger and 1C gap, the biplane configuration with 0C stagger and $1 \mathrm{C}$ gap (introduced in section 5.3) and the equivalent monoplane configuration (introduced in section 5.4 and 6.1.2). The equivalent monoplane had the same reference surface area (Lift capability) as the biplane joined at the tips with endplates and

$\checkmark$ The same span: $b_{\text {monoplane }}=b_{\text {biplane_with_endplates }}$ and therefore different AR

$\checkmark$ The same total load

$\checkmark$ The same structural material

$\checkmark$ Constant thickness along the span and the chord

$\checkmark \quad$ Elliptical lift load distribution

The criteria chosen for this class of aircraft are the following: Weight/Structural performance, Portability, Safety, Aerodynamic performance, Reliability, Low visibility. These criteria reflect the criteria listed in the specifications of the requirement section in Chapter III. 


\begin{tabular}{|l|c|c|c|c|c|c|c|}
\hline Criteria & Weight & \multicolumn{2}{l|}{ Eq. Monoplane } & Biplane & \multicolumn{2}{l|}{ Joined at the } \\
tips with \\
endplates
\end{tabular}

Table 12 Decision analysis: In order to make a final choice and proceed with the following design steps, a decision analysis was performed. \% Criteria were chosen and 3 configuration analyzed. The criteria reflects the specifications expressed in the requirements of Chapter III. The most important criteria selected was the weight. The final score shows a slightly greater score for the joined at the tips configuration.

The criteria chosen for this class of aircraft are the following (as reported in Table 12): Weight/Structural performance, Portability, Safety, Aerodynamic performance, Reliability, Low visibility. These criteria reflect the criteria listed in the specifications of the requirements section in Chapter III.

Due to the potential use of the proposed aircraft in surveillance missions, the weight of the UAV must be consistent with the physical resistance and strength of a soldier to transport it during a battle and to throw it for the launch. Therefore this 
criterion was selected as the most important. From sections 5.3 and 5.4, it appears evident that the use of the box configuration helps reduce the weight of the structure in comparison to the monoplane and biplane configurations.

On the other side, due to the increased number of wing pieces, the box configuration got a lower score for the portability criteria. Portability is very important for this class of aircraft because they have to be transported and assembled easily anywhere that surveillance is necessary.

The next criterion considered is the aerodynamic performance. An increase in the endurance of the UAV is desirable in order to extend the time this UAV performs its main functional requirement. The potential decrease in weight of the box configuration in comparison to the other 2 configurations, provides the possibility of increasing the autonomy of the battery, increasing therefore the endurance of the airplane, keeping the same motor and mission profile.

Due to the expensive payload carried by the aircraft, due also to the important data stored during the mission by the aircraft and due to the same high technology product this UAV represents, it is desirable to design a reliable aircraft capable of accomplishing several missions without failures and without being caught by the enemy. With regard to these criteria, the conventional monoplane configuration has a better rank because of the greater knowledge developed in the last 50 years, that excludes unexpected surprises. After the monoplane, the biplane remains more affordable than the completely new box configuration.

Finally, due to its low target altitude, the aircraft has to be a low profile visibility airplane, to avoid the possibility of being detected by enemies. The larger chord of the monoplane with respect to the other configurations makes it somehow bigger and visible but the use of 2 wings of the biplane and box configuration make 
these configuration more visible in perspective. The same rank was therefore given to all three configurations for these criteria.

The overall sum shows a greater score for the configuration joined at the tips, with 59 points, followed by the monoplane configuration with 58 points and the biplane with 55. There is not a big gap among all of them and in particular between the monoplane and the boxplane. Due to this small gap, that can potentially decrease to 0 or even be in favour of the monoplane configuration if some of the assumptions fall, the final best choice for the design configuration remains the use of a conventional monoplane configuration, unless some other advantages are found to sustain the position of the wing box configuration. 


\section{Chapter VII}

\section{Conclusions and future work}

This research demonstrates that the nonplanar configuration under investigation can meet the requirements of a Small Reconnaissance Surveillance and Target Acquisition UAV listed in Chapter III.

The conceptual design for the required mission profile of a small UAV was therefore completed in Chapter IV.

An effort was made to try to understand if there are any structural and/or aerodynamic advantages when compared to a standard monoplane or biplane configuration. The following conclusions can be made: under the following assumptions:

$\checkmark$ An elliptical load distribution along the quarter chord

$\checkmark$ A symmetrical lift distribution between the upper and the lower wing

$\checkmark$ The same reference area between the nonplanar wing concept under investigation and the equivalent wing configuration

$\checkmark$ The same total load

$\checkmark$ The same structural material

$\checkmark$ Constant thickness along the span and the chord 
- The nonplanar wing concept under investigation can potentially meet some of the requirements of a Small Reconnaissance Surveillance and Target Acquisition UAV

$\checkmark$ Light weight due to the potential reduction in structural weight

$\checkmark$ Hand Launched due to the potential reduction of in stall speed

$\checkmark$ Potential increased Endurance, using the same battery and motor

$\checkmark$ Low visibility and portability due to the small size of the span in comparison to an equivalent monoplane with a span two times bigger.

- Using the finite element software ADINA, eight structural models with varying gap and stagger were created from the nonplanar wing concept under investigation. The model with 1c gap and 0 stagger has the smallest Maximum Effective Stress at the root. The stagger has a deteriorating effect on the structural behaviour of the wing while the gap and therefore the endplates at the tips of the wing have a beneficial structural effect.

- If the model with 1c gap and 0 stagger is compared to an equivalent biplane, under the above-mentioned assumptions, a reduction of $32.03 \%$ in the maximum stress is noticed, with an increase in the weight of $13.5 \%$ due to the presence of the endplates.

- If the model with $1 \mathrm{c}$ gap and 0 stagger is compared to an equivalent monoplane, under the above assumptions and the further assumptions of the same AR, different span and the same maximum effective stress at the root, a 
potential weight saving of $30 \%$ is realized, with a reduction in the stall speed of $19 \%$ and an increase in the endurance $6.4 \%$, using the same battery capacity and the same motor.

- Using the panel vortex code AVL a second equivalent monoplane model was created under the assumptions of inviscid, steady, incompressible and irrotational flow. If the model with $1 \mathrm{c}$ gap and 0 stagger is compared to this second equivalent monoplane, under the additional assumptions of the same span, different $\mathrm{AR}$ and the same maximum effective stress at the root, a potential weight saving of $3.5 \%$ is realized. From an aerodynamic point of view, an increase in the lift slope is also noticed. Besides for small angles of attack, 0 thru $4^{\circ}$, the values of Lift to Drag ratio, that represent an estimate of the aerodynamic efficiency of an aircraft, are greater than that for the equivalent monoplane. In particular at the supposed design $C_{L}$ for the Small UAV, a gain in $\mathrm{L} / \mathrm{D}$ of $17 \%$ is realized.

Despite these advantages, from the decision analysis performed in Chapter VI, considering the criteria listed in the specifications requirements in Chapter III:

- Weight

- Portability

- Aerodynamic Performance

- Reliability

- Low visibility

And comparing 3 possible layouts: 
- Boxplane nonplar configuration

- Standard biplane

- Eq. monoplane

the overall score showed a not too clear advantage for the nonplanar configuration joined at the tips and therefore a conventional standard monoplane configuration is suggested.

These results encourage potential future work in order to

- Understand if from a stability, control and aeroelastic point of view there are any other advantages that can increase the potential advantages of the use of the joined at the tips configuration over the monoplane configuration

- Step through further iterations in the design process of the Small UAV 